\title{
An Emergent Autonomous Flow for Mean-Field Spin Glasses
}

\author{
James MacLaurin*
}

February 24, 2021

\begin{abstract}
We study the dynamics of symmetric and asymmetric spin-glass models of size $N$. The analysis is in terms of the double empirical process: this contains both the spins, and the field felt by each spin, at a particular time (without any knowledge of the correlation history). It is demonstrated that in the large $N$ limit, the dynamics of the double empirical process becomes deterministic and autonomous over finite time intervals. This does not contradict the well-known fact that SK spin-glass dynamics is nonMarkovian (in the large $N$ limit) because the empirical process has a topology that does not discern correlations in individual spins at different times. In the large $N$ limit, the evolution of the density of the double empirical process approaches a nonlocal autonomous PDE operator $\Phi_{t}$. Because the emergent dynamics is autonomous, in future work one will be able to apply PDE techniques to analyze bifurcations in $\Phi_{t}$. Preliminary numerical results for the SK Glauber dynamics suggest that the 'glassy dynamical phase transition' occurs when a stable fixed point of the flow operator $\Phi_{t}$ destabilizes.
\end{abstract}

\section{Introduction}

This paper studies the emergent dynamics of mean-field non-spherical spin-glasses. At low temperature, spin-glass systems are characterized by slow emergent timescales that typically diverge with the system size (see [41, 62, 44] for good surveys of known results). Probably the most famous mean-field spin glass model is that of Sherrington and Kirkpatrick [58]. It is widely known in the physics community that the SK spin glass undergoes a 'dynamical phase transition' as the temperature is lowered [4, 5, 41, 62]. Essentially what this means is that the average correlation-in-time of spins does not go to zero as time progresses: that is, some spins get locked into particular states and flip extremely rarely. Although there has been much progress in the study of spin glass dynamics $[9,40,10]$, a rigorous proof of a dynamical phase transition in the original SK spin glass model remains elusive. More precisely, although it is known that the time to equilibrium is $O(1)$ when the temperature $\beta^{-1}$ is high $[8,33]$, there is lacking a proof that the time-to-equilibrium diverges with $N$ when $\beta$ is large (to the best of this author's knowledge). Furthermore it is well-established that the equilibrium SK Spin-Glass system undergoes a 'Replica Symmetry Breaking' phase transition as $\beta$ increases $[39,64,55]$, and this leads many scholars to expect that a phase transition should also be manifest in the initial dynamics. The equilibrium 'Replica Symmetry Breaking' transition is characterized by the distribution of the overlap between two independent replica not concentrating at 0 , but possessing a continuous density over an interval away from zero [53,65]. A major reason for the lack of a rigorous characterization of the dynamical phase transition (as emphasized by Ben Arous [5] and Guionnet [41]) is that the existing large $N$ emergent equations are not autonomous and very difficult to analyze rigorously. This paper takes steps towards this goal by deriving an autonomous PDE for the emergent (large $N$ ) dynamics: this PDE should be more amenable to a bifurcation analysis (to be performed in future work) than the existing nonautonomous delay equations $[9,37]$. These results are also of great relevance to the dynamics of asymmetric spin glass models, which have seen a resurgence of interest in neuroscience in recent years [30, 28, 29, 47, 26, 24, 32].

This paper determines the emergent dynamics of $M$ 'replica' spin glass systems started at initial conditions that are independent of the connections. 'Replicas' means that we take identical copies of the same static

\footnotetext{
*New Jersey Institute of Technology. james.n.maclaurin@njit.edu
} 
connection topology $\mathbf{J}$, and conditionally on $\mathbf{J}$, run independent and identically-distributed jump-Markov stochastic processes on each replica. As noted above, Replicas are known to shed a lot of insight into the rich tree-like structure of 'pure states' that emerge in the static SK spin glass at low temperature $[53,39,64,65,55]$, and it is thus reasonable to conjecture that replicas will shed much insight into the dynamical phase transition. Indeed Ben Arous and Jagannath [6] use the overlap of two replicas to determine bounds on the spectral gap determining the rate of convergence to equilibrium of mean-field spin glasses. Writing $\mathcal{E}=\{-1,1\}$, the spins flip between -1 and 1 at rate $c\left(\sigma_{t}^{i, j}, G_{t}^{i, j}\right)$ for some general function $c$ : $\{-1,1\} \times \mathbb{R} \rightarrow \mathbb{R}^{+}$, where the field felt by the spin is written as

$$
G_{t}^{i, j}=N^{-\frac{1}{2}} \sum_{k=1}^{N} J^{j k} \sigma_{t}^{i, k}
$$

and $\mathbf{J}=\left\{J^{j k}\right\}_{1 \leq j \leq k \leq N}$ are i.i.d. centered Gaussian variables with a specified level of symmetry. For Glauber dynamics for the SK spin glass [53], the connections are symmetric (i.e. $J^{j k}=J^{k j}$ ) and the dynamics is reversible, with $c$ taking the form [38],

$$
c(\sigma, g)=(1+\exp \{2 \beta \sigma(g+h)\})^{-1},
$$

where $h$ is a constant known as the magnetization, and $\beta^{-1}$ is the temperature. In this case, the spin-glass dynamics are reversible with respect to the following Gibbs Measure

$$
\mu_{\beta, \mathbf{J}}^{N}(\boldsymbol{\sigma})=\exp \left(\frac{\beta}{2} \sum_{p=1}^{M} \sum_{j, k=1}^{N} J^{j k} \sigma^{p, j} \sigma^{p, k}+h \sum_{p=1}^{M} \sum_{j=1}^{N} \tilde{\sigma}^{p, j}-N M \rho_{\mathbf{J}}^{N}\right)
$$

where $\rho_{\mathbf{J}}^{N}$ is a normalizing factor, often called the free energy, given by

$$
\rho_{\mathbf{J}}^{N}=N^{-1} \log \sum_{\boldsymbol{\sigma} \in \mathcal{E}^{N}}\left[\exp \left(\frac{\beta}{2} \sum_{j, k=1}^{N} J^{j k} \sigma^{j} \sigma^{k}+h \sum_{j=1}^{N} \sigma^{j}\right)\right] .
$$

For further details on the equilibrium Gibbs measure, see the reviews in $[13,65,55]$. It is known that as $\beta$ increases from 0 , a sharp transition occurs, where the convergence to equilibrium bifurcates from being $O(1)$ in time, to timescales that diverge in $N[5,44]$.

One of the novelties of this paper is to study the emergent properties of the double empirical process $\left(\hat{\mu}_{t}^{N}(\boldsymbol{\sigma}, \mathbf{G})\right)_{t \geq 0}$, which contains information on the distribution of the spins and fields, without knowledge of the 'history' of each spin and field. Formally, $\hat{\mu}^{N}(\boldsymbol{\sigma}, \mathbf{G})$ is a càdlàg $\mathcal{P}$-valued process (where $\mathcal{P}=\mathcal{M}_{1}^{+}\left(\mathcal{E}^{M} \times\right.$ $\left.\mathbb{R}^{M}\right)$ ), i.e.

$$
\begin{aligned}
\hat{\mu}^{N}(\boldsymbol{\sigma}, \mathbf{G}) & : \mathcal{D}([0, \infty), \mathcal{E})^{M N} \times \mathcal{D}([0, \infty), \mathbb{R})^{M N} \rightarrow \mathcal{D}([0, \infty), \mathcal{P}), \\
\hat{\mu}^{N}(\boldsymbol{\sigma}, \mathbf{G}) & :=\left\{\hat{\mu}^{N}\left(\boldsymbol{\sigma}_{t}, \mathbf{G}_{t}\right)\right\}_{t \in[0, \infty)} \text { where } \\
\hat{\mu}^{N}\left(\boldsymbol{\sigma}_{t}, \mathbf{G}_{t}\right) & =N^{-1} \sum_{j \in I_{N}} \delta_{\left(\sigma_{t}^{1, j}, \ldots, \sigma_{t}^{M, j}\right),\left(G_{t}^{1, j}, \ldots, G_{t}^{M, j}\right)}
\end{aligned}
$$

where $\left\{\sigma_{t}^{i, j}\right\}$ is the solution of the jump Markov Process, and the fields are defined in (1).

We now overview some of the existing literature on the dynamics of the SK spin glass. In the physics literature, averaging over quenched disorder been used to derive limiting equations for the correlation functions $[61,60,42,59,53,22,49,48]$. The first rigorous mathematical results were obtained in the seminal work of Ben Arous and Guionnet $[9,11]$ (these results were for a similar 'soft-spin' model driven by Brownian Motions). Guionnet [40] expanded on this work to prove that in the soft SK spin glass started at i.i.d initial conditions, the dynamics of the empirical measure converges to a unique limit, with no restriction on time or temperature. Grunwald [37, 38] obtained analogous equations for the limiting dynamics of the pathwise empirical measure for the jump-Markov system studied in this paper. More recent work by Ben 
Arous, Dembo and Guionnet has rigorously established the Cugliandolo-Kurchan [22] / Crisanti-HornerSommers [21] equations for spherical spin glasses using Gaussian concentration inequalities [10]. A recent preprint of Dembo, Lubetzky and Zeitouni has established universality for asymmetric spin glass dynamics, extending the work of Ben Arous and Guionnet to non-Gaussian connections, with no restriction on time or temperature [24].

In the papers cited above, the emergent large $N$ dynamics is non-autonomous: that is, one needs to know the full history of the emergent variable (either the empirical measure, or correlation / response functions) upto time $t$ to predict the dynamics upto time $t+\delta t$. In the early work of Ben Arous, Guionnet and Grunwald $[9,40,11,37]$, the emergent variable is the pathwise empirical measure. This is an extremely rich object because it 'knows' about average correlations in individual spins at different times. Ben Arous and Guionnet [9] demonstrated that the limiting dynamics of the pathwise empirical measure is the law of a complicated implicit delayed stochastic differential equation. In the later work of Ben Arous, Dembo and Guionnet on spherical spin glasses, a simpler set of emergent variables was used: the correlation and response functions $[4,10]$ (this formalism is frequently used by physicists $[42,53,22]$ ). In the $p=2$ case, the resultant equations are autonomous, and this allowed them to rigorously prove that there is a dynamical phase transition [4].

There is still lacking a rigorous characterization of the dynamical phase transition in the non-spherical SK model. As has been emphasized by Ben Arous [5] and Guionnet [41], a fundamental difficulty is that all of the known emergent equations are non-autonomous (that is, they are either delay integro-differential equations, or an implicit delayed SDE [9,37]). A major reason that the emergent equations are not autonomous is that the emergent object studied by $[9,37]$ - the pathwise empirical measure - carries too much information, because it knows about the history of the spin-flipping. This is why this paper focuses on determining the limiting dynamics of a different order parameter: the double empirical process (as defined in (5)-(7)) that cannot discern time-correlations in individual spins. The empirical process carries more information about the system than that of Ben Arous, Guionnet [9, 40,11] and Grunwald [37] insofar as it contains information about overlaps between different replicas, but less information insofar as it does not know about correlationsin-time of individual spins. The chief advantage of working with this order parameter is that the dynamics becomes autonomous in the large $N$ limit, just as in classical methods for studying the empirical process in interacting particle systems [23, 63]. One can now apply the apparatus of PDEs to the limiting equations to study the bifurcation of the fixed points. Indeed preliminary analytic work has identified that there is a bifurcation in the fixed point of the flow (31) for SK Glauber dynamics, and 2 replica (see Remark 2.5).

Many recent applications of dynamical spin glass theory have been in neuroscience, being referred to as networks of balanced excitation and inhibition. Typically the connections in these networks are almost asymmetric, unlike in the original SK model. These applications include networks driven by white noise $[17,14,66,16,30,28,29,31]$ and also deterministic disordered networks $[1,47,26,20]^{1}$; the common element to all of these papers being the random connectivity of mean zero and high variance. It has been argued that the highly variable connectivity in the brain is a vital component to the emergent gamma rhythm [14]. Another important application of spin-glass theory has been the study of stochastic gradient descent algorithms $[7,54]$.

Our fundamental result is to show that as $N \rightarrow \infty$, the empirical process converges to have a density given by a Mckean-Vlasov-type $\mathrm{PDE}^{2}$ of the form, for $\boldsymbol{\alpha} \in \mathcal{E}^{M}$ and $\mathrm{x} \in \mathbb{R}^{M}$,

$$
\frac{\partial p_{t}}{\partial t}(\boldsymbol{\alpha}, \mathbf{x})=\sum_{i=1}^{M}\left\{c\left(-\alpha^{i}, x^{i}\right) p_{t}(\boldsymbol{\alpha}[i], \mathbf{x})-c\left(\alpha^{i}, x^{i}\right) p_{t}(\boldsymbol{\alpha}, \mathbf{x})+2 L_{i i}^{\xi_{t}} \frac{\partial^{2} p_{t}}{\partial x_{i}^{2}}(\boldsymbol{\alpha}, \mathbf{x})\right\}-\nabla \cdot\left\{\mathbf{m}^{\xi_{t}}(\boldsymbol{\alpha}, \mathbf{x}) p_{t}(\boldsymbol{\alpha}, \mathbf{x})\right\},
$$

where $\xi_{t} \in \mathcal{M}_{1}^{+}\left(\mathcal{E}^{M} \times \mathbb{R}^{M}\right)$ is the probability measure with density $p_{t}, \boldsymbol{\alpha}[i]$ is the same as $\boldsymbol{\alpha}$, except that the $i^{t h}$ spin has a flipped sign. $\mathbf{m}^{\xi_{t}}$ and $\mathbf{L}^{\xi_{t}}$ are functions defined in Section 2.

In broad outline, our method of proof resembles that of Ben Arous and Guionnet [9] and Grunwald [37], insofar as (i) we freeze the interaction and (ii) study the Gaussian properties of the field variables. However our approach is different insofar as, after freezing the interaction, we do not use Girsanov's Theorem to

\footnotetext{
${ }^{1}$ One should be able to adapt the methods of this paper to this setting.

${ }^{2}$ See $[23,63]$ for further discussion of such PDEs.
} 
study a tilted system, but instead study the pathwise evolution of the empirical process over small time increments. This pathwise approach to the Large Deviations of interacting particle systems has been popular in recent years: being employed in the work of Budhiraja, Dupuis and colleagues [15], in this author's work on interacting particle systems with a sparse random topology [52], and subsequent work in [18, 31, 19]. More precisely, we study the evolution over small time intervals of the expectation of test functions with respect to the double empirical measure: a method that has been applied to interacting particle systems in, for example, [45] and [51]. To understand the change in the fields $\left\{G_{t}^{j}\right\}$ over a small increment in time, we use the law $\gamma$ of the connections, conditioned on the value of the fields at that time step. It is fundamental to our proof that - essentially due to the Woodbury formula for the inverse of a matrix with a finite-rank perturbation - the conditional Gaussian density can be written as a function of the empirical measure $\hat{\mu}_{t}^{N}(\boldsymbol{\sigma}, \mathbf{G})=N^{-1} \sum_{j \in I_{N}} \delta_{\left(\boldsymbol{\sigma}_{t}^{j}, \mathbf{G}_{t}^{j}\right)}$ and the local spin and field variables (see the analysis in Section 7.1).

Notation: Let $\mathcal{E}=\{-1,1\}$. For any Polish Space $\mathcal{X}$, we let $\mathcal{M}_{1}^{+}(\mathcal{X})$ denote all probability measures on $\mathcal{X}$, and $\mathcal{D}([0, T], \mathcal{X})$ the Skorohod space of all $\mathcal{X}$-valued càdlàg functions [12]. We always endow $\mathcal{M}_{1}^{+}(\mathcal{X})$ with the topology of weak convergence. Let $\mathcal{P}:=\mathcal{M}_{1}^{+}\left(\mathcal{E}^{M} \times \mathbb{R}^{M}\right)$ denote the set of all probability measures on $\mathcal{E}^{M} \times \mathbb{R}^{M}$, and define the subset

$$
\tilde{\mathcal{P}}=\left\{\mu \in \mathcal{P}: \mathbb{E}^{\mu}\left[\|x\|^{2}\right]<\infty\right\} .
$$

For any vector $\mathbf{g} \in \mathbb{R}^{M},\|\mathbf{g}\|$ is the Euclidean norm, and $\|\mathbf{g}\|_{\infty}$ is the supremum norm. For any square matrix $\mathbf{K} \in \mathbb{R}^{m \times m},\|\mathbf{K}\|$ is the operator norm, i.e.

$$
\|\mathbf{K}\|=\sup _{\mathbf{x} \in \mathbb{R}^{m}:\|\mathbf{x}\|=1}\{\|\mathbf{K} \mathbf{x}\|\} .
$$

Let $d_{W}$ be the Wasserstein Metric $[63,34]$ on $\tilde{\mathcal{P}}$, i.e.

$$
d_{W}(\beta, \zeta)=\inf _{\eta}\left\{\mathbb{E}^{\eta}[\|\mathbf{x}-\mathbf{g}\|+\|\boldsymbol{\alpha}-\boldsymbol{\sigma}\|]\right\} .
$$

where the infimum is over all measures $\eta \in \mathcal{M}_{1}^{+}\left(\mathcal{E}^{M} \times \mathbb{R}^{M} \times \mathcal{E}^{M} \times \mathbb{R}^{M}\right)$ with marginals $\beta$ (over the first two variables), and $\zeta$ (over the second two variables). We let $\mathcal{C}([0, T], \mathcal{X})$ denote the space of all continuous functions from $[0, T]$ to $\mathcal{X}$. $\mathcal{B}(\mathcal{X})$ denotes the Borelian subsets.

The spins are indexed by $I_{N}:=\{1,2, \ldots, N\}$, and the replicas by $I_{M}:=\{1,2, \ldots, M\}$. The typical indexing convention that we follow is $\boldsymbol{\sigma}_{t}^{j}=\left(\sigma_{t}^{1, j}, \ldots, \sigma_{t}^{M, j}\right)^{T} \in \mathcal{E}^{M}$, and $\boldsymbol{\sigma}_{t}=\left(\sigma_{t}^{i, j}\right)_{i \in I_{M}, j \in I_{N}} \in \mathcal{E}^{N M}$.

\section{Outline of model and main result}

Let $\left(\Omega, \mathcal{F},\left(\mathcal{F}_{t}\right), \mathbb{P}\right)$ be a filtered probability space containing the following random variables. The connections $\left(J^{j k}\right)_{j, k \in \mathbb{Z}}$ are centered Gaussian random variables, with joint law $\gamma \in \mathcal{M}_{1}^{+}\left(\mathbb{R}^{\mathbb{Z}^{+} \times \mathbb{Z}^{+}}\right)$. To lighten the notation we assume that there are self-connections (one could easily extend the results of this paper to the case where there are no self-connections). Their covariance is taken to be of the form

$$
\mathbb{E}^{\gamma}\left[J^{j k} J^{l m}\right]=\delta(j-l) \delta(k-m)+\mathfrak{s} \delta(j-m) \delta(k-l) .
$$

The parameter $\mathfrak{s} \in[0,1]$ is a constant indicating the level of symmetry in the connections. In the case that $\mathfrak{s}=1, J^{j k}=J^{k j}$ identically, and in the case that $\mathfrak{s}=0, J^{j k}$ is probabilistically independent of $J^{k j}$. (One could easily extend these results to the case that $\mathfrak{s} \in[-1,0)) .\left\{J^{j k}\right\}_{j, k \in \mathbb{Z}^{+}}$are assumed to be $\mathcal{F}_{0}$-measurable.

We take $M$ replicas of the spins: this means that the connections $\mathbf{J}$ are the same across the different systems, but (conditionally on $\mathbf{J}$ ) the spin-jumps in different systems are independent. Our reason for working with replicas is that, as discussed in the introduction, in the case of reversible dynamics, replicas are known to shed much light on the rich 'tree-like' structure of pure states in the equilibrium Gibbs measure $[56,53,39,64,55]$. If one wishes to avoid replicas, one could just take $M=1$. The spins $\left\{\sigma_{t}^{i, j}\right\}_{j \in I_{N}, i \in I_{M}, t \geq 0}$ 
constitute a system of jump Markov processes: $i$ being the replica index, and $j$ being the spin index. Spin $(i, j)$ flips between states in $\mathcal{E}=\{-1,1\}$ with intensity $c\left(\sigma_{t}^{i, j}, G_{t}^{i, j}\right)$ (where $G_{t}^{i, j}=N^{-\frac{1}{2}} \sum_{k=1}^{N} J^{j k} \sigma_{t}^{i, k}$ ) for a function $c: \mathcal{E} \times \mathbb{R} \rightarrow[0, \infty)$ for which we make the following assumptions:

- $c$ is strictly positive and uniformly bounded, i.e. for some constant $c_{1}>0$,

$$
\sup _{\sigma \in \mathcal{E}} \sup _{g \in \mathbb{R}} \mid\left(c(\sigma, g) \mid \leq c_{1} \text { and } c(\sigma, g)>0 .\right.
$$

- The following Lipschitz condition is assumed: for a constant $c_{L}>0$, for all $\sigma \in \mathcal{E}$ and $g_{1}, g_{2} \in \mathbb{R}$,

$$
\begin{aligned}
\left|c\left(\sigma, g_{1}\right)-c\left(\sigma, g_{2}\right)\right| & \leq c_{L}\left|g_{1}-g_{2}\right| \\
\left|\log c\left(\sigma, g_{1}\right)-\log c\left(\sigma, g_{2}\right)\right| & \leq c_{L}\left|g_{1}-g_{2}\right| .
\end{aligned}
$$

- The following limits exist for $\sigma= \pm 1$,

$$
\lim _{g \rightarrow-\infty} c(\sigma, g), \lim _{g \rightarrow \infty} c(\sigma, g) .
$$

- The $\log$ of $c$ is bounded in the following way: there exists a constant $C_{g}>0$ such that

$$
\sup _{\alpha \in \mathcal{E}}|\log c(\alpha, g)| \leq C_{g}|g| \text {. }
$$

We note that the Glauber Dynamics for the reversible dynamics in (2) satisfy the above assumptions [35, 38].

To facilitate the proofs, we represent the stochasticity as a time-rescaled system of Poisson counting processes of unit intensity [27]. We thus define $\left\{Y^{i, j}(t)\right\}_{i \in I_{M}, j \in \mathbb{Z}^{+}}$to be independent Poisson processes, which are also independent of the disorder variables $\left\{J^{j k}\right\}_{j, k \in \mathbb{Z}^{+}}$. We define the spin system $\left\{\sigma_{t}^{i, j}\right\}$ to be the unique solution of the following system of SDEs

$$
\sigma_{t}^{i, j}=\sigma_{0}^{i, j} \times A \cdot Y^{i, j}\left(\int_{0}^{t} c\left(\sigma_{s}^{i, j}, G_{s}^{i, j}\right) d s\right),
$$

where $A \cdot x:=(-1)^{x}$. Clearly $\sigma_{t}^{i, j}$ depends on $N$ (for convenience this is omitted from the notation). The law of the initial condition $\sigma_{0}$ is written as $\mu_{0} \in \mathcal{M}_{1}^{+}\left(\mathcal{E}^{M N}\right)$. $\mu_{0}$ is assumed to be independent of the disorder. Note that the forward Komolgorov equation describing the dynamics of the law $P_{\mathbf{J}}^{N}(t) \in \mathcal{M}_{1}^{+}\left(\mathcal{E}^{M N}\right)$ of the spins at time $t$ (conditioned on a realization $\mathbf{J}$ of the disorder) is [27]

$$
\frac{d P_{\mathbf{J}}^{N}(\boldsymbol{\sigma})}{d t}=\sum_{i \in I_{M}, j \in I_{N}}\left\{c\left(-\sigma_{t}^{i, j}, \hat{G}_{t}^{i, j}\right) P_{\mathbf{J}}^{N}(\boldsymbol{\sigma}[i, j])-c\left(\sigma_{t}^{i, j}, G_{t}^{i, j}\right) P_{\mathbf{J}}^{N}(\boldsymbol{\sigma})\right\},
$$

where $\boldsymbol{\sigma}[i, j] \in \mathcal{E}^{M N}$ is the same as $\boldsymbol{\sigma}$, except that the spin with indices $(i, j)$ has a flipped sign, and $\hat{G}_{t}^{i, j}=N^{-1 / 2} \sum_{k \in I_{N}, k \neq j} J^{j k} \sigma_{t}^{i, k}-N^{-1 / 2} J^{j j} \sigma_{t}^{i, j}$.

For some fixed constant $\mathfrak{c}>0$, define the set

$$
\mathcal{X}^{N}=\left\{\boldsymbol{\eta} \in \mathcal{E}^{N M}: \inf _{\mathfrak{b} \in \mathbb{R}^{M}:\|\mathfrak{b}\|=1} \sum_{p, q \in I_{M}, j \in I_{N}} \eta^{p, j} \eta^{q, j} \mathfrak{b}^{p} \mathfrak{b}^{q}>N \mathfrak{c}\right\} .
$$

We assume that the initial condition is such that

$$
\varlimsup_{N \rightarrow \infty} N^{-1} \log \mathbb{P}\left(\sigma_{0} \notin \mathcal{X}^{N}\right)<0 .
$$

Note that (20) is satisfied if $\left\{\boldsymbol{\sigma}_{0}^{j}\right\}_{j \in I_{N}}$ are iid samples from some probability law $\tilde{\mu}_{0} \in \mathcal{M}_{1}^{+}\left(\mathcal{E}^{M}\right)$ that is such that

$$
\inf _{\mathfrak{b} \in \mathbb{R}^{M}:\|\mathfrak{b}\|=1} \mathbb{E}^{\tilde{\mu}_{0}}\left[\langle\mathfrak{b}, \boldsymbol{\sigma}\rangle^{2}\right]>\mathfrak{c} .
$$


One would then find that (20) follows from Sanov's Theorem [25]. For an arbitrary positive constant $T>0$, we define

$$
\tau_{N}=T \wedge \inf \left\{t: t \in[0, T] \text { and } \boldsymbol{\sigma}_{t} \notin \mathcal{X}^{N}\right\}
$$

If $\tau_{N}<T$, then the smallest eigenvalue of the overlap matrix $\mathbf{K}^{\hat{\mu}_{\tau_{N}}^{N}}$ (as defined in (24)) is c. Intuitively, the stopping time is reached when the spins in different replicas are too similar. One expects that this is an extremely rare event, even on timescales diverging in $N$. See Remark 2.4. The main result of this paper is the following. We emphasize that these are 'quenched' results. 'Annealing' methods are not used in this paper.

Theorem 2.1. Fix $T>0$. There exists a flow operator $\Phi: \mathcal{P} \rightarrow \mathcal{C}([0, T], \mathcal{P})$ written $\Phi \cdot \mu:=\left\{\Phi_{t} \cdot \mu\right\}_{t \geq 0}$ such that $\Phi_{0} \cdot \mu=\mu$ and for any $\epsilon>0$

$$
\varlimsup_{N \rightarrow \infty} N^{-1} \log \mathbb{P}\left(\sup _{t \leq \tau_{N}} d_{W}\left(\Phi_{t} \cdot \hat{\mu}^{N}\left(\boldsymbol{\sigma}_{0}, \mathbf{G}_{0}\right), \hat{\mu}^{N}\left(\boldsymbol{\sigma}_{t}, \mathbf{G}_{t}\right)\right) \geq \epsilon\right)<0 .
$$

The flow $\Phi$ is specified in Section 2.1. It follows immediately from the Borel-Cantelli Theorem that $\mathbb{P}$ almost surely

$$
\lim _{N \rightarrow \infty} \sup _{t \leq \tau_{N}} d_{W}\left(\Phi_{t} \cdot \hat{\mu}^{N}\left(\boldsymbol{\sigma}_{0}, \mathbf{G}_{0}\right), \hat{\mu}^{N}\left(\boldsymbol{\sigma}_{t}, \mathbf{G}_{t}\right)\right)=0
$$

\subsection{Existence and Uniqueness of the Flow $\Phi_{t}$}

In this section we define $\Phi \cdot \mu \in \mathcal{C}([0, T], \mathcal{P})$, for any $\mu \in \mathcal{P}$ such that $\mathbb{E}^{\mu(\sigma, g)}\left[g^{2}\right]<\infty$. We write $\Phi \cdot \mu:=$ $\left\{\Phi_{t} \cdot \mu\right\}_{t \in[0, T]}$, where $\Phi_{t}: \mathcal{P} \rightarrow \mathcal{P}$, and in the following we write $\xi_{t}=\Phi_{t} \cdot \mu$.

Lemma 2.2. Fix $T>0$. For any $\mu \in \mathcal{P}:=\mathcal{M}_{1}^{+}\left(\mathcal{E}^{M} \times \mathbb{R}^{M}\right)$ such that $\mathbb{E}^{\mu(\sigma, g)}\left[g^{2}\right]<\infty$, there exists a unique set of measures $\left\{\xi_{t}\right\}_{t \in[0, T]} \subset \mathcal{P}$ with the following characteristics

1. For all $t \in(0, T], \xi_{t}$ has a density in its second variable, i.e. $d \xi_{t}(\boldsymbol{\sigma}, \mathbf{x})=p_{t}(\boldsymbol{\sigma}, \mathbf{x}) d \mathbf{x} . p_{t}(\boldsymbol{\sigma}, \mathbf{x})$ is continuously differentiable in $t$, twice continuously differentiable in $\mathbf{x}$, and satisfies the system of equations (24)- (31).

2. $\xi_{0}=\mu$, and for all $t \in[0, T], t \rightarrow \xi_{t}$ is continuous.

For any $\xi \in \mathcal{P}$ such that $\mathbb{E}^{\xi(\sigma, g)}\left[\|g\|^{2}\right]<\infty$, define the $M \times M$ coefficient matrices $\left\{\mathbf{L}^{\xi}, \boldsymbol{\kappa}^{\xi}, \boldsymbol{v}^{\xi}, \mathbf{K}^{\xi}\right\} \in \mathbb{R}^{M^{2}}$ to have the following elements,

$$
\begin{aligned}
K_{j k}^{\xi} & =\mathbb{E}^{\xi(\boldsymbol{\sigma}, \mathbf{x})}\left[\sigma^{j} \sigma^{k}\right] \\
L_{j k}^{\xi} & =\mathbb{E}^{\xi(\boldsymbol{\sigma}, \mathbf{x})}\left[\sigma^{k} \sigma^{j} c\left(\sigma^{j}, x^{j}\right)\right] \\
\kappa_{j k}^{\xi} & =\mathbb{E}^{\xi(\boldsymbol{\sigma}, \mathbf{x})}\left[x^{k} \sigma^{j} c\left(\sigma^{j}, x^{j}\right)\right] \\
v_{j k}^{\xi} & =\mathbb{E}^{\xi(\boldsymbol{\sigma}, \mathbf{x})}\left[\sigma^{k} x^{j}\right] .
\end{aligned}
$$

For any $\mu \in \mathcal{P}$, define $\Lambda^{\mu}$ to be the smallest eigenvalue of $\mathbf{K}^{\mu}$, i.e.

$$
\Lambda^{\mu}=\inf _{\mathfrak{a} \in \mathbb{R}^{M}:\|\mathfrak{a}\|=1} \sum_{j, k=1}^{M} K_{j k}^{\mu} \mathfrak{a}^{j} \mathfrak{a}^{k}=\inf _{\mathfrak{a} \in \mathbb{R}^{M}:\|\mathfrak{a}\|=1} \mathbb{E}^{\mu}\left[\left(\sum_{j=1}^{M} \mathfrak{a}^{j} \sigma^{j}\right)^{2}\right],
$$

noting that the eigenvalues of $\mathbf{K}^{\mu}$ are real (since it is symmetric) and non-negative. To facilitate the following proofs (in particular, the existence and uniqueness of the solution to the PDE), we want the following functions $\mathbf{m}^{\xi}(\boldsymbol{\sigma}, \mathbf{x})$ and $\mathbf{L}^{\xi}$ to be uniformly Lipschitz for all $\xi \in \mathcal{P}$. Indeed thanks to our definition of the stopping time $\tau_{N}$, it does not matter how $\mathbf{m}^{\xi}$ is defined for $\xi$ such that $\Lambda^{\xi}<\mathfrak{c} / 2$, as long as $\epsilon$ is sufficiently small. To this end, we choose a definition that ensures that $\xi \rightarrow \mathbf{H}^{\xi}$ is uniformly Lipschitz, i.e.

$$
\mathbf{H}^{\xi}=\left\{\begin{array}{l}
\left(\mathbf{K}^{\xi}\right)^{-1} \text { if } \Lambda^{\xi} \geq \mathfrak{c} / 2 \\
\left(\mathbf{I}\left(\mathfrak{c} / 2-\Lambda^{\xi}\right)+\mathbf{K}^{\xi}\right)^{-1} \text { otherwise. }
\end{array}\right.
$$


Now define the vector field $\mathbf{m}^{\xi}(\boldsymbol{\sigma}, \mathbf{x}): \mathcal{P} \times \mathcal{E}^{M} \times \mathbb{R}^{M} \rightarrow \mathbb{R}^{M}$ as follows

$$
\mathbf{m}^{\xi}(\boldsymbol{\sigma}, \mathbf{x})=-2 \mathbf{L}^{\xi} \mathbf{H}^{\xi} \mathbf{x}-2 \mathfrak{s} \boldsymbol{\kappa}^{\xi} \mathbf{H}^{\xi} \boldsymbol{\sigma}+2 \mathfrak{s} \mathbf{L}^{\xi} \mathbf{H}^{\xi} \boldsymbol{v}^{\xi} \mathbf{H}^{\xi} \boldsymbol{\sigma} .
$$

We can now write down the PDE that defines the density of $\xi_{t}:=\Phi_{t}(\mu)$. For some $\boldsymbol{\alpha} \in \mathcal{E}^{M}$ and $\mathbf{x} \in \mathbb{R}^{M}$, we write $p_{t}(\boldsymbol{\alpha}, \mathbf{x})$ to be the density of $\xi_{t}$ in its second variable, i.e. $\xi_{t}\left(\boldsymbol{\sigma}=\boldsymbol{\alpha}, g^{i} \in\left[x^{i}, x^{i}+d x^{i}\right]\right):=$ $p_{t}(\boldsymbol{\alpha}, \mathbf{x}) d x^{1} \ldots d x^{M}$. Write $\boldsymbol{\alpha}[i] \in \mathcal{E}^{M}$ to be almost identical to $\boldsymbol{\alpha}$, except that the $i^{\text {th }}$ spin has a flipped sign. The evolution of the densities is governed by the following system of partial differential equations

$$
\frac{\partial p_{t}}{\partial t}(\boldsymbol{\alpha}, \mathbf{x})=\sum_{i \in I_{M}}\left\{c\left(-\alpha^{i}, x^{i}\right) p_{t}(\boldsymbol{\alpha}[i], \mathbf{x})-c\left(\alpha^{i}, x^{i}\right) p_{t}(\boldsymbol{\alpha}, \mathbf{x})+2 L_{i i}^{\xi_{t}} \frac{\partial^{2} p_{t}}{\partial\left(x^{i}\right)^{2}}(\boldsymbol{\alpha}, \mathbf{x})\right\}-\nabla \cdot\left\{\mathbf{m}^{\xi_{t}}(\boldsymbol{\alpha}, \mathbf{x}) p_{t}(\boldsymbol{\alpha}, \mathbf{x})\right\} .
$$

Remark 2.3. We emphasize that the convergence result in Theorem 2.1 does not hold for the path-wise empirical measure, i.e.

$$
\tilde{\mu}^{N}=N^{-1} \sum_{j \in I_{N}} \delta_{\left(\sigma_{[0, T]}^{j}, G_{[0, T]}^{j}\right)} \in \mathcal{M}_{1}^{+}\left(\mathcal{D}\left([0, T], \mathcal{E}^{M} \times \mathbb{R}^{M}\right)\right)
$$

endowed with the Skorohod topology on the set of càdlàg paths $\mathcal{D}\left([0, T], \mathcal{E}^{M}\right)$ [27]. Indeed it is known that the limit of the pathwise empirical measure is non-Markovian, so the Markovian stochastic hybrid system with Fokker-Planck equation given by (31) is almost certainly not the limiting law for the pathwise empirical measure [9]. This does not mean that our result in Theorem 2.1 is inconsistent with the non-Markovian results in the work of Ben Arous, Guionnet and Grunwald [9, 37], since the topology in our theorem cannot discern correlations in particular spins at different times.

Remark 2.4. It seems plausible that for any temperature $\beta>0$ and any $T>0$, there exists $\mathfrak{c}$ such that

$$
\varlimsup_{N \rightarrow \infty} N^{-1} \log \mathbb{P}\left(\tau_{N}<T\right)<0 .
$$

Perhaps one could prove this by demonstrating that the attracting manifold of the flow $\Phi_{t}$ is such that all eigenvalues of $\mathbf{K}^{\xi_{t}}$ are strictly positive. One expects this to be true because of the presence of the diffusions in the PDE. However the author has not yet seen an easy proof of this.

Remark 2.5. Suppose that the dynamics is reversible, with spin-flipping intensity given by $(2), h=0$ and the symmetry $\mathfrak{s}=1$. Preliminary numerical work by C.MacLaurin ${ }^{3}$ has identified a family of fixed point solutions to (8) with two replicas (i.e. $M=2$ ). Let $q \geq 0$ satisfy the implicit relationship

$$
\begin{aligned}
& \frac{1+q}{1-q}-\cosh (2 \beta h) \exp \left(2 \beta^{2} q\right)=0 \text { and define the matrix elements } \\
& K_{11}^{\xi}=K_{22}^{\xi}=1 \text { and } K_{12}^{\xi}=K_{21}^{\xi}=q \\
& v_{11}^{\xi}=v_{22}^{\xi}=\frac{\beta}{2}\left(1+q^{2}\right) \text { and } v_{12}^{\xi}=v_{21}^{\xi}=\beta q \text { and } \kappa_{i j}^{\xi}=0 .
\end{aligned}
$$

With the above definitions, the field distributions $p(\boldsymbol{\alpha}, \cdot)$ in the fixed point solution to (8) are weighted Gaussians. For $h=0$, there is a bifurcation as $\beta$ increases through 1 in the solutions to (32): for $\beta \leq 1, q=0$ is the unique solution, but for $\beta>1$, it is no longer unique.

\subsection{Proof Outline}

We discretize time into $(n+1)$ timesteps $\left\{t_{a}^{(n)}\right\}_{0 \leq a \leq n}$ : writing $\Delta=t_{a+1}^{(n)}-t_{a}^{(n)}=T n^{-1}$. In Section 3 we use an argument that is reminiscent of Gronwall's Inequality to demonstrate that if the action of the flow operator over the time interval $\left[t_{a}^{(n)}, t_{a+1}^{(n)}\right]$ corresponds to the dynamics of the empirical process to within an error of $o(\Delta)$,

\footnotetext{
${ }^{3}$ Private communication.
} 
then the supremum of the difference between the empirical process and the flow over the entire interval $[0, T]$ must be small. We also introduce an approximate flow $\Psi_{t}$, obtained by evaluating the coefficients in the PDE at $\hat{\mu}_{t}^{N}$ rather than $\xi_{t}$. In subsequent sections it will be easier to compare $\Psi_{t}$ to $\hat{\mu}_{t}^{N}$ than to compare $\Phi_{t}$ to $\hat{\mu}_{t}^{N}$.

To accurately estimate the 'average' change in the fields $G_{t_{a}^{(n)}}^{q, j} \rightarrow G_{t_{a+1}^{(n)}}^{q, j}$ we must perform a change-ofmeasure to a stochastic process $\tilde{\sigma}_{i, t}^{q, j}$ whose spin-flipping is independent of the connections. The reason for this change of measure is that now the changed fields $\tilde{G}_{t}^{i, j}:=N^{-1 / 2} \sum_{k \in I_{N}} J^{j k} \tilde{\sigma}_{t}^{i, k}$ are Gaussian, and their incremental behavior can be accurately predicted by studying their covariance structure. In Section 4 we define $C_{\mathfrak{n}}^{N}$ such processes $\left\{\tilde{\boldsymbol{\sigma}}_{i, t}\right\}_{1 \leq i \leq C_{\mathfrak{n}}^{N}}$, and we demonstrate that the probability law of the original $\mathcal{E}^{M N_{-}}$ valued process $\boldsymbol{\sigma}_{t}$ must be close to at least one of them using Girsanov's Theorem. The partition of the path space $\mathcal{D}\left([0, T], \mathcal{E}^{M}\right)^{N}$ is implemented using a second, finer, discretization of time into $\left\{t_{a}^{(m)}\right\}_{0 \leq a \leq m}$, for some $m$ which is an integer multiple of $n$. This finer partition of time is needed to ensure that the Girsanov exponent is sufficiently close to unity.

In Section 5 we demonstrate that the Wasserstein distance can be approximated arbitrarily well by taking the supremum of the difference in expectation of a finite set of smooth functions. Working now exclusively with the processes $\tilde{\boldsymbol{\sigma}}_{i, t}$, we Taylor expand the change in expectation of such functions from $t_{a}^{(n)}$ to $t_{a+1}^{(n)}$, for both the empirical measure and the flow operator $\Phi_{t}$. The Taylor expansion implies that only the first two moments of the empirical measure and flow operator need to match in order that the change in the Wasserstein Distance is $o(\Delta)$. There are two basic types of term in the difference of the Taylor Expansions: (i) terms that can be bounded using concentration inequalities for Poisson Processes $\left\{Y^{q, j}(t)\right\}_{q \in I_{M}, j \in I_{N}}$, and (ii) terms that require the law $\gamma$ of the Gaussian connections $\left\{J^{j k}\right\}_{j, k \in I_{N}}$ to be accurately bounded.

In Section 6, we bound the terms (i) whose dynamics can be accurately predicted using the Law of Large numbers for Poisson Processes. These bounds typically involve concentration inequalities for compensated Poisson Processes (which are Martingales [2]). In Section 7, we bound the terms (ii), using the conditional Gaussian probability law $\gamma_{\tilde{\boldsymbol{\sigma}}, \tilde{\mathbf{G}}}$ - obtained by taking the law $\gamma \in \mathcal{M}_{1}^{+}\left(\mathbb{R}^{N^{2}}\right)$ of the connections $\left\{J^{j k}\right\}_{j, k \in I_{N}}$ and conditioning on the values of the NM field variables $\left\{\tilde{G}_{t_{a}^{(n)}}^{q, j}\right\}_{q \in I_{M}, j \in I_{N}}$. We demonstrate that the average change in the field terms $\tilde{G}_{t_{a+1}^{(n)}}^{q, j}-\tilde{G}_{t_{a}^{(n)}}^{q, j}$ is governed by the first and second moments of $\gamma_{\boldsymbol{\sigma}, \mathbf{G}}$. The first moment ultimately leads to the term $\mathbf{m}^{\xi_{t}}$ in (31), and the second moment ultimately leads to the diffusion coefficient $\sqrt{L_{i i}^{\xi_{t}}}$

Before we commence the above plan, we require that the flow operator $\Phi_{t}$ is well defined.

Proof of Lemma 2.2. We can interpret $p_{t}$ as the marginal probability law of the solution of a nonlinear SDE driven by a Levy Process. [46] proved the existence and uniqueness of a solution to such an SDE in the case that the coefficients are uniformly Lipschitz functions of the probability law (with respect to the Wasserstein distance). By contrast, our coefficients $\mathbf{m}^{\xi_{t}}$ and $\left(\mathbf{L}^{\xi_{t}}\right)^{1 / 2}$ (one must take the square root of the diffusion coefficient to obtain the coefficient of the stochastic integral) are only locally Lipschitz (see Lemma 2.6).

To get around this, one first uses [46] to show existence and uniqueness for an analogous system driven by uniformly Lipschitz coefficients $\hat{\mathbf{m}}^{\xi_{t}}$ and $\left\{\left(\hat{L}_{i i}^{\xi_{t}}\right)^{1 / 2}\right\}_{i \in I_{M}}$. These coefficients are taken to be identical to $\mathbf{m}^{\xi_{t}}$ and $\left(L_{i i}^{\xi_{t}}\right)^{1 / 2}$ when $\xi_{t} \in \mathcal{D}_{\epsilon}$, where

$$
\mathcal{D}_{\epsilon}=\left\{\mu \in \mathcal{P}: \sup _{i \in I_{M}} \mathbb{E}^{\mu}\left[\left(x^{i}\right)^{2}\right] \leq \epsilon^{-1} \text { and } \inf _{i \in I_{M}} \mathbb{E}^{\mu}\left[c\left(\alpha^{i}, x^{i}\right)\right] \geq \epsilon\right\} .
$$

The solution is written as $\xi_{\epsilon, t}$. One then shows that for small enough $\epsilon, \xi_{\epsilon, t} \in \mathcal{D}_{\epsilon}$ for all $t \in[0, T]$. Once one has shown this, it must be that $\xi_{t}:=\xi_{\epsilon, t}$ is the unique solution.

To do this, one can easily show (analogously to Lemma 3.6) that for all $\epsilon>0$, there exist constants $C_{1}, C_{2}>0$ such that

$$
\frac{d}{d t} \mathbb{E}^{\xi_{\epsilon, t}}\left[\left(x^{i}\right)^{2}\right] \leq C_{1} \mathbb{E}^{\xi_{\epsilon, t}}\left[\left(x^{i}\right)^{2}\right]+C_{2}
$$


The boundedness of $\mathbb{E}^{\xi_{\epsilon, t}}\left[\left(x^{i}\right)^{2}\right]$ then implies a lower bound for $\hat{L}_{i i, t}^{\xi_{\epsilon, t}}$, since for any $u>0$, thanks to Chebyshev's Inequality, $\xi_{\epsilon, t}\left(\left|x^{i}\right| \leq u\right) \geq 1-\mathbb{E}^{\xi_{\epsilon, t}}\left[\left(x^{i}\right)^{2}\right] u^{-2}$, and the continuity of $c$ implies that $\inf _{|x| \leq u, \sigma \in \mathcal{E}} c(\sigma, x)>0$. Since $\mu \rightarrow L_{i i}^{\mu}$ is uniformly Lipschitz, it must be that $\mu \rightarrow D_{i i}^{\mu}$ is uniformly Lipschitz over $\mathcal{D}_{\epsilon}$, since $L_{i i}^{\mu}$ is bounded away from zero.

The above existence and uniqueness proof requires that the coefficients of the PDE in (31) are Lipschitz. This is noted in the follow Lemma.

Lemma 2.6. (i) There exists a constant $C_{1}>0$ such that for any $\beta, \zeta \in \tilde{\mathcal{P}}$,

$$
\begin{aligned}
& \sup _{1 \leq p, q \leq M}\left|L_{p q}^{\beta}-L_{p q}^{\zeta}\right|,\left|K_{p q}^{\beta}-K_{p q}^{\zeta}\right| \leq C_{1} d_{W}(\beta, \zeta) \\
& \sup _{1 \leq p, q \leq M}\left|v_{p q}^{\beta}-v_{p q}^{\zeta}\right|,\left|\kappa_{p q}^{\beta}-\kappa_{p q}^{\zeta}\right| \leq C_{1}\left(1+\mathbb{E}^{\beta}\left[\|\mathbf{x}\|^{2}\right]^{\frac{1}{2}}\right) d_{W}(\beta, \zeta) .
\end{aligned}
$$

(ii) There is a constant $C>0$ such that for all $\beta, \zeta \in \tilde{\mathcal{P}}$ such that $\Lambda^{\beta}, \Lambda^{\zeta} \geq \mathfrak{c} / 2$, all $\boldsymbol{\alpha}, \boldsymbol{\sigma} \in \mathcal{E}^{M}$ and all $\mathbf{x}, \mathbf{g} \in \mathbb{R}^{M}$,

$$
\begin{aligned}
\left\|\mathbf{m}^{\beta}(\boldsymbol{\alpha}, \mathbf{x})-\mathbf{m}^{\zeta}(\boldsymbol{\sigma}, \mathbf{g})\right\| & \leq C d_{W}(\beta, \zeta)\left\{1+\|\mathbf{g}\|+\mathbb{E}^{\zeta}\left[\|\mathbf{g}\|^{2}\right]^{\frac{1}{2}}\right\}+C\|\mathbf{x}-\mathbf{g}\|+C\left\{1+\mathbb{E}^{\zeta}\left[\|\mathbf{g}\|^{2}\right]^{\frac{1}{2}}\right\}\|\boldsymbol{\alpha}-\boldsymbol{\sigma}\| \\
\left\|\mathbf{m}^{\beta}(\boldsymbol{\alpha}, \mathbf{g})\right\| & \leq C\|\mathbf{g}\|+C\left(1+\mathbb{E}^{\beta}\left[\|\mathbf{g}\|^{2}\right]^{\frac{1}{2}}\right) .
\end{aligned}
$$

Proof. Both results follow almost immediately from the definitions, since $|c(\cdot, \cdot)|$ is uniformly bounded, and $|c(\alpha, x)-c(\alpha, g)| \leq c_{L}|x-g|$. It follows from the definition in (29) that $\xi \rightarrow H_{j k}^{\xi}$ is uniformly Lipschitz (for all indices $j, k \in I_{M}$ ), since (as noted in (i) of this lemma) $\xi \rightarrow K_{j k}^{\xi}$ is uniformly Lipschitz. Furthermore $\left|H_{j k}^{\xi}\right|$ is uniformly bounded, because $\left|K_{j k}^{\xi}\right| \leq 1$.

\section{Organization of Proof of Theorem 2.1}

This section lays the groundwork for the proof of Theorem 2.1, using an argument that is reminiscent of Gronwall's Inequality. The ultimate aim of this section is to demonstrate that, if the change in the empirical process over a small increment $\Delta$ in time is similar to the incremental change induced by the flow operator $\Phi_{\Delta} \cdot \hat{\mu}_{t}^{N}$, then the distance $\sup _{t \in[0, T]} d_{W}\left(\hat{\mu}_{t}^{N}, \Phi_{t} \cdot \hat{\mu}^{N}\right)$ is $O\left(\Delta^{2}\right)$. Thus this section reduces the proof of Theorem 2.1, to the sufficient condition in Lemma 3.5. The rest of the paper is then oriented towards proving Lemma 3.5. The proofs of the lemmas stated just below are deferred to later in the section.

We will express the event in the statement of Theorem 2.1 as a union of $a_{N}$ subevents, i.e.

$$
\left\{\sup _{t \leq \tau_{N}} d_{W}\left(\Phi_{t} \cdot \hat{\mu}^{N}\left(\boldsymbol{\sigma}_{0}, \mathbf{G}_{0}\right), \hat{\mu}^{N}\left(\boldsymbol{\sigma}_{t}, \mathbf{G}_{t}\right)\right) \geq \epsilon\right\} \subseteq \bigcup_{j=1}^{a_{N}} \mathcal{A}_{j}^{N} .
$$

As is noted in the following lemma, it will then suffice to show that the probability of each of the subevents $\left\{\mathcal{A}_{j}^{N}\right\}$ is exponentially decaying.

Lemma 3.1. Suppose that events $\left\{\mathcal{A}_{j}^{N}\right\}_{j=1}^{a_{N}}$ are such that $\varlimsup_{N \rightarrow \infty} N^{-1} \log a_{N}=0$. Then

$$
\varlimsup_{N \rightarrow \infty} N^{-1} \log \mathbb{P}\left(\bigcup_{j=1}^{a} \mathcal{A}_{j}^{N}\right) \leq \varlimsup_{N \rightarrow \infty} \sup _{1 \leq j \leq a_{N}}\left\{N^{-1} \log \mathbb{P}\left(\mathcal{A}_{j}^{N}\right)\right\}
$$

Proof. Immediate from the definitions. 
We now outline more precisely what these events are. First, we require that the matrix of connections is sufficiently regular. Let $\mathbf{J}_{N}$ be the $N \times N$ matrix with $(j, k)$ element equal to $N^{-\frac{1}{2}} J^{j k}$. Define $\mathcal{J}_{N}$ to be the event

$$
\begin{aligned}
& \mathcal{J}_{N}=\left\{\left\|\mathbf{J}_{N}\right\| \leq 3\right\} \text { and } \\
& \mathcal{W}_{2}=\left\{\mu \in \mathcal{P}: \sup _{1 \leq p \leq M} \mathbb{E}^{\mu(\boldsymbol{\sigma}, \mathbf{g})}\left[\left(g^{p}\right)^{2}\right] \leq 3\right\} \text { and } \mathcal{W}_{2, \mathfrak{c}}=\left\{\mu \in \mathcal{W}_{2}: \inf _{\mathfrak{a} \in \mathbb{R}^{M:\|\mathfrak{a}\|=1}} \sum_{j, k=1}^{M} K_{j k}^{\mu} a^{j} a^{k} \geq \mathfrak{c}\right\} .
\end{aligned}
$$

The following lemma notes that $\mathcal{J}_{N}$ is overwhelmingly likely.

Lemma 3.2. 1 .

$$
\varlimsup_{N \rightarrow \infty} N^{-1} \log \gamma\left(\mathcal{J}_{N}^{c}\right):=\Lambda_{J}<0
$$

2. Also,

$$
\mathcal{J}_{N} \subseteq\left\{\text { For all } t \geq 0, \hat{\mu}_{t}^{N} \in \mathcal{W}_{2}\right\}
$$

Define the spaces of measures

$$
\begin{aligned}
& \mathcal{W}_{[0, T]}=\left\{\mu_{[0, T]} \in \mathcal{D}([0, T], \mathcal{P}): \mu_{t} \in \mathcal{W}_{2} \text { and } t \rightarrow \mu_{t} \text { has finite \# discontinuities }\right\} \\
& \hat{\mathcal{W}}_{[0, T]}=\left\{\mu_{[0, T]} \in \mathcal{W}_{[0, T]}: \sup _{t \in[0, T], p \in I_{M}} \mathbb{E}^{\mu_{t}}\left[\left\|\mathbf{x}^{p}\right\|^{2}\right] \leq 3\right\} .
\end{aligned}
$$

Next we define a map $\Psi: \mathcal{W}_{[0, T]} \rightarrow \mathcal{C}([0, T], \mathcal{P}), \Psi:=\left(\Psi_{t}\right)_{t \in[0, T]}$, that is an approximation of the flow $\Phi_{t}$, such that the coefficients of the PDE are evaluated at $\hat{\mu}_{t}^{N}$, rather than $\xi_{t}$. More precisely, it is such that $\Psi \cdot \mu_{[0, T]}:=\eta_{[0, T]}$, and for $t>0, \eta_{t}$ has density $p_{t}$ satisfying the PDE

$$
\frac{\partial p_{t}}{\partial t}(\boldsymbol{\alpha}, \mathbf{x})=\sum_{i \in I_{M}}\left\{c\left(-\alpha^{i}, x^{i}\right) p_{t}(\boldsymbol{\alpha}[i], \mathbf{x})-c\left(\alpha^{i}, x^{i}\right) p_{t}(\boldsymbol{\alpha}, \mathbf{x})+2 L_{i i}^{\mu_{t}} \frac{\partial^{2} p_{t}}{\partial x_{i}^{2}}(\boldsymbol{\alpha}, \mathbf{x})\right\}-\nabla \cdot\left\{\mathbf{m}^{\mu_{t}} p_{t}(\boldsymbol{\alpha}, \mathbf{x})\right\}
$$

We insist that $\eta_{0}=\mu_{0}$, and that $t \rightarrow \eta_{t}$ is continuous. Write $\Psi_{t} \cdot \mu_{[0, T]}:=\eta_{t}$. One can easily check that $\Psi$ is uniquely defined.

The following lemma states that $\Psi$ is a good approximation of $\Phi$. The second result in the lemma is necessary for us to be sure that we avoid the pathological situation of $\Lambda_{t}^{\hat{\mu}_{t}^{N}} \rightarrow 0$, which would mean that the coefficients in the PDE blowup (see the definition in (29)). Incidentally, this is precisely the reason that we require the stopping time $\tau_{N}$ in $(21)$.

Lemma 3.3. Define $\tilde{d}_{T}: \mathcal{D}([0, T], \mathcal{P}) \times \mathcal{D}([0, T], \mathcal{P}) \rightarrow \mathbb{R}^{+}$to be

$$
\tilde{d}_{T}\left(\mu_{[0, T]}, \nu_{[0, T]}\right)=\sup _{t \in[0, T]} d_{W}\left(\mu_{t}, \nu_{t}\right)
$$

noting that $\tilde{d}_{T}$ does not metrize the Skorohod topology. For any $\epsilon>0$, there exists $\delta>0$ such that

$$
\left\{\mu \in \hat{\mathcal{W}}_{[0, T]}: \tilde{d}_{T}(\Psi \cdot \mu, \mu)<\delta\right\} \subseteq\left\{\mu \in \hat{\mathcal{W}}_{[0, T]}: \tilde{d}_{T}\left(\Phi \cdot \mu_{0}, \mu\right)<\epsilon\right\}
$$

Furthermore, there exists $\delta_{\mathfrak{c}}$ such that for all $\delta \leq \delta_{\mathfrak{c}}$,

$$
\mathbf{H}^{\Psi_{t} \cdot \hat{\mu}_{b}^{N}}=\left(\mathbf{K}^{\Psi_{t} \cdot \hat{\mu}_{b}^{N}}\right)^{-1} \text { as long as } t<\tau_{N} \text { and } d_{W}\left(\Psi_{t} \cdot \hat{\mu}^{N}, \hat{\mu}^{N}\left(\boldsymbol{\sigma}_{t}, \mathbf{G}_{t}\right)\right) \leq \delta .
$$

Next we discretize time, and also the flow $\Psi_{t}$. We partition the time interval $[0, T]$ into $\left\{t_{b}^{(n)}\right\}_{b=0}^{n-1}$, with $t_{b}^{(n)}=b \Delta$ and $\Delta=T / n$. For any $t \in[0, T]$, define $t^{(n)}:=\sup \left\{t_{b}^{(n)}: t_{b}^{(n)} \leq t\right\}$. We write $\Psi_{b}:=\Psi_{t_{b}^{(n)}}$, $\hat{\mu}_{b}^{N}(\boldsymbol{\sigma}, \mathbf{G}):=\hat{\mu}_{t_{b}^{(n)}}^{N}, \boldsymbol{\sigma}_{b}:=\boldsymbol{\sigma}_{t_{b}^{(n)}}$. 
We can now decompose the event in the statement of Theorem 2.1 into the following events. It follows from Lemma 3.3 that for any $\tilde{\epsilon}>0$, there must exist $\epsilon>0$ such that

$$
\begin{aligned}
\left\{\sup _{t \leq \tau_{N}} d_{W}\left(\Phi_{t} \cdot \hat{\mu}_{0}^{N}, \hat{\mu}_{t}^{N}\right) \geq \tilde{\epsilon}\right\} \subseteq & \left\{\sup _{t \leq \tau_{N}} d_{W}\left(\Psi_{t} \cdot \hat{\mu}^{N}, \hat{\mu}_{t}^{N}\right) \geq \epsilon\right\} \\
\subseteq & \mathcal{J}_{N}^{c} \cup \bigcup_{0 \leq b \leq n}\left\{\mathcal{J}_{N} \text { and } \sup _{t \in\left[t_{b}^{(n)} \wedge \tau_{N}, t_{b+1}^{(n)} \wedge \tau_{N}\right]} d_{W}\left(\Psi_{t} \cdot \hat{\mu}^{N}, \Psi_{b} \cdot \hat{\mu}^{N}\right) \geq \epsilon / 3\right\} \cup \\
& \bigcup_{0 \leq b \leq n}\left\{\mathcal{J}_{N} \text { and } \sup _{t \in\left[t_{b}^{(n)} \wedge \tau_{N}, t_{b+1}^{(n)} \wedge \tau_{N}\right]} d_{W}\left(\hat{\mu}_{t}^{N}, \hat{\mu}_{b}^{N}\right) \geq \epsilon / 3\right\} \cup \\
& \bigcup_{0 \leq b \leq n}\left\{\mathcal{J}_{N} \text { and for some } b \text { such that } \tau_{N}>t_{b}^{(n)}, d_{W}\left(\hat{\mu}_{b}^{N}, \Psi_{b} \cdot \hat{\mu}^{N}\right) \geq \epsilon / 3\right\} .
\end{aligned}
$$

It is assumed that $\epsilon \leq \delta_{\mathfrak{c}}$, as defined in Lemma 3.3. Thanks to Lemma 3.1, for Theorem 2.1, to hold, it thus suffices to prove that some $n \in \mathbb{Z}^{+}$,

$$
\begin{array}{r}
\sup _{0 \leq b<n} \varlimsup_{N \rightarrow \infty} N^{-1} \log \mathbb{P}\left(\mathcal{J}_{N} \text { and } \sup _{t \in\left[t_{b}^{(n)} \wedge \tau_{N}, t_{b+1}^{(n)} \wedge \tau_{N}\right]} d_{W}\left(\Psi_{t} \cdot \hat{\mu}^{N}, \Psi_{b} \cdot \hat{\mu}^{N}\right) \geq \epsilon / 3\right)<0 \\
\sup _{0 \leq b<n} \varlimsup_{N \rightarrow \infty} N^{-1} \log \mathbb{P}\left(\mathcal{J}_{N} \text { and } \sup _{t \in\left[t_{b}^{(n)} \wedge \tau_{N}, t_{b+1}^{(n)} \wedge \tau_{N}\right]} d_{W}\left(\hat{\mu}_{t}^{N}, \hat{\mu}_{b}^{N}\right) \geq \epsilon / 3\right)<0 \\
\varlimsup_{N \rightarrow \infty} N^{-1} \log \mathbb{P}\left(\mathcal{J}_{N} \text { and for some } b \text { such that } \tau_{N}>t_{b}^{(n)}, d_{W}\left(\hat{\mu}_{b}^{N}, \Psi_{b} \cdot \hat{\mu}^{N}\right) \geq \epsilon / 3\right)<0 \\
\varlimsup_{N \rightarrow \infty} N^{-1} \log \mathbb{P}\left(\mathcal{J}_{N}^{c}\right)<0 .
\end{array}
$$

(47) is demonstrated in Lemma 3.6, (48) is established in Lemma 3.7 and(50) is a consequence of Lemma 3.2 .

In order that Theorem 2.1 is true, it thus only remains to prove (49). Define the events $\left\{\mathcal{U}_{b}^{N}\right\}_{b=0}^{n-1}$, for a positive constant $\mathfrak{u}>0$ (to be specified more precisely below - for the moment we note that $\mathfrak{u}$ will be chosen independently of $n$ and $N$ ), and writing $\tilde{\epsilon}=\epsilon / 3$,

$$
\mathcal{U}_{b}^{N}=\left\{\mathcal{J}_{N}, d_{W}\left(\Psi_{b+1} \cdot \hat{\mu}^{N}, \hat{\mu}_{b+1}^{N}\right)>\tilde{\epsilon} \exp \left(\mathfrak{u} t_{b+1}^{(n)} / T-\mathfrak{u}\right), d_{W}\left(\Psi_{b} \cdot \hat{\mu}^{N}, \hat{\mu}_{b}^{N}\right) \leq \tilde{\epsilon} \exp \left(\mathfrak{u} t_{b}^{(n)} / T-\mathfrak{u}\right) \text { and } \tau_{N}>t_{b}^{(n)}\right\},
$$

and observe that

$$
\left\{\mathcal{J}_{N} \text { and for some } b \text { such that } \tau_{N}>t_{b}^{(n)}, d_{W}\left(\hat{\mu}_{b}^{N}, \Psi_{b} \cdot \hat{\mu}^{N}\right)>\tilde{\epsilon}\right\} \subseteq \bigcup_{b=0}^{n-1} \mathcal{U}_{b}^{N} .
$$

We thus find from Lemma 3.1 that, in order that (49) holds, it suffices to prove that

$$
\sup _{0 \leq b<n} \varlimsup_{N \rightarrow \infty} N^{-1} \log \mathbb{P}\left(\mathcal{U}_{b}^{N}\right)<0 .
$$

We now make a further approximation to the operator $\Psi_{t}$. For any $\boldsymbol{\sigma} \in \mathcal{E}^{M N}$ and $\mathbf{G} \in \mathbb{R}^{M N}$, define the random measure $\xi_{b}(\boldsymbol{\sigma}, \mathbf{G}) \in \mathcal{P}$, which is such that $\xi_{b}(\boldsymbol{\sigma}, \mathbf{G}) \simeq \Psi_{b+1} \cdot \hat{\mu}^{N}(\boldsymbol{\sigma}, \mathbf{G})$, as follows. Let $\left\{\tilde{Y}^{p}(t)\right\}_{p \in I_{M}}$ be independent Poisson Counting Processes, and $\left\{\tilde{W}_{t}^{p}\right\}_{p=1}^{M}$ independent Wiener Processes (they are also independent of the proceses $Y^{p, j}(t)$ and connections $\mathbf{J}$ used to define the original system). Writing $\hat{\mu}_{b}^{N}(\boldsymbol{\sigma}, \mathbf{G})$ to be the law of random variables $\left(\boldsymbol{\zeta}_{0}, \mathbf{x}_{0}\right)$, define $\xi_{b}(\boldsymbol{\sigma}, \mathbf{G})$ to be the law of $\left(\boldsymbol{\zeta}_{\delta t}, \mathbf{x}_{\delta t}\right)$, where, recalling that $A \cdot x:=(-1)^{x}$, for each $p \in I_{M}$,

$$
\begin{aligned}
\zeta_{\delta t}^{p} & =\zeta_{0}^{p} A \cdot \tilde{Y}^{p}\left(\Delta c\left(\zeta_{0}^{p}, x_{0}^{p}\right)\right) \\
\mathbf{x}_{\delta t} & =\mathbf{x}_{0}+\delta t \mathbf{m}^{\hat{\mu}_{b}^{N}}\left(\boldsymbol{\zeta}_{0}, \mathbf{x}_{0}\right)+\mathbf{D}^{\hat{\mu}_{b}^{N}} \tilde{\mathbf{W}}_{\delta t}, \text { where } D_{i j}^{\hat{\mu}_{b}^{N}}=2 \sqrt{L_{i i}^{\hat{\mu}_{b}^{N}}} \delta(i, j),
\end{aligned}
$$

and $\Delta=T / n$. When the context is clear, we omit the argument of $\xi_{b}$. 
It follows from the facts that (i) $d_{W}\left(\Psi_{b+1} \cdot \hat{\mu}^{N}, \hat{\mu}_{b+1}^{N}\right) \leq d_{W}\left(\xi_{b}, \hat{\mu}_{b+1}^{N}\right)+d_{W}\left(\Psi_{b+1} \cdot \hat{\mu}^{N}, \xi_{b}\right)$ and (ii) $\exp \left(\mathfrak{u} t_{b+1}^{(n)} / T-\right.$ $\mathfrak{u}) \geq \exp \left(\mathfrak{u} t_{b}^{(n)} / T+\mathfrak{u} \Delta / 2 T-\mathfrak{u}\right)+\exp \left(\mathfrak{u} t_{b}^{(n)} / T-\mathfrak{u}\right) \mathfrak{u} \Delta / 2 T$ (recalling that $\left.\Delta=t_{b+1}^{(n)}-t_{b}^{(n)}\right)$, that

$$
\begin{aligned}
\left\{d_{W}\right. & \left.\left(\Psi_{b+1} \cdot \hat{\mu}^{N}, \hat{\mu}_{b+1}^{N}\right)>\tilde{\epsilon} \exp \left(\mathfrak{u} t_{b+1}^{(n)} / T-\mathfrak{u}\right)\right\} \subseteq\left\{d_{W}\left(\xi_{b}, \hat{\mu}_{b+1}^{N}\right)>\exp \left(\mathfrak{u} t_{b}^{(n)} / T-\mathfrak{u}\right) \tilde{\epsilon} \mathfrak{u} \Delta / 2 T\right\} \\
& \cup\left\{d_{W}\left(\Psi_{b+1} \cdot \hat{\mu}^{N}, \xi_{b}\right)>\exp \left(\mathfrak{u} t_{b}^{(n)} / T+\mathfrak{u} \Delta / 2 T-\mathfrak{u}\right) \tilde{\epsilon} \text { and } d_{W}\left(\xi_{b}, \hat{\mu}_{b+1}^{N}\right) \leq \exp \left(\mathfrak{u} t_{b}^{(n)} / T-\mathfrak{u}\right) \tilde{\epsilon} \mathfrak{u} \Delta / 2 T\right\} \\
& \left\{d_{W}\left(\xi_{b}, \hat{\mu}_{b+1}^{N}\right)>\exp \left(\mathfrak{u} t_{b}^{(n)} / T-\mathfrak{u}\right) \tilde{\epsilon} \mathfrak{u} \Delta / 2 T\right\} \cup\left\{d_{W}\left(\Psi_{b+1} \cdot \hat{\mu}^{N}, \xi_{b}\right)>\exp \left(\mathfrak{u} t_{b}^{(n)} / T+\mathfrak{u} \Delta / 2 T-\mathfrak{u}\right) \tilde{\epsilon}\right\}
\end{aligned}
$$

We thus find that

$$
\begin{aligned}
& \mathcal{U}_{b}^{N} \subseteq\left\{\mathcal{J}_{N} \text { and } d_{W}\left(\xi_{b}, \hat{\mu}_{b+1}^{N}\right)>\exp \left(\mathfrak{u} t_{b}^{(n)} / T-\mathfrak{u}\right) \tilde{\epsilon} \mathfrak{u} \Delta /(2 T)\right\} \bigcup \\
& \left\{\mathcal{J}_{N} \text { and } d_{W}\left(\Psi_{b+1} \cdot \hat{\mu}^{N}, \xi_{b}\right)>\tilde{\epsilon} \exp \left(\mathfrak{u} t_{b}^{(n)} / T+\mathfrak{u} \Delta /(2 T)-\mathfrak{u}\right) \text { and } d_{W}\left(\Psi_{b} \cdot \hat{\mu}^{N}, \hat{\mu}_{b}^{N}\right) \leq \exp \left(\mathfrak{u} t_{b}^{(n)} / T-\mathfrak{u}\right) \tilde{\epsilon}\right\} .
\end{aligned}
$$

Therefore (52) will be seen to be true once we demonstrate Lemmas 3.4 and 3.5.

Lemma 3.4. For any $\tilde{\epsilon}>0$, for all sufficiently large $n$, and all $b$ such that $0 \leq b<n$,

$$
\begin{array}{r}
\varlimsup_{N \rightarrow \infty} N^{-1} \log \mathbb{P}\left(\mathcal{J}_{N} \text { and } \tau_{N}>t_{b+1}^{(n)} \text { and } d_{W}\left(\Psi_{b+1} \cdot \hat{\mu}^{N}, \xi_{b}\right)>\tilde{\epsilon} \exp \left(\mathfrak{u} t_{b}^{(n)} / T+\mathfrak{u} \Delta / 2 T-\mathfrak{u}\right)\right. \\
\text { and } \left.d_{W}\left(\Psi_{b} \cdot \hat{\mu}^{N}, \hat{\mu}_{b}^{N}\right) \leq \tilde{\epsilon} \exp \left(\mathfrak{u} t_{b}^{(n)} / T-\mathfrak{u}\right)\right)<0 .
\end{array}
$$

Lemma 3.4 is proved later in this section.

Lemma 3.5. Suppose that for any $\bar{\epsilon}>0$, for all sufficiently large $n$ and all $0 \leq b \leq n-1$,

$$
\varlimsup_{N \rightarrow \infty} N^{-1} \log \mathbb{P}\left(\mathcal{J}_{N} \text { and } \tau_{N}>t_{b}^{(n)} \text { and } d_{W}\left(\xi_{b}, \hat{\mu}_{b+1}^{N}\right) \geq \bar{\epsilon} \Delta\right)<0 .
$$

Then Theorem 2.1 must be true.

The rest of this paper is devoted to establishing Lemma 3.5. In the next section, Lemma 4.6 determines a sufficient condition for Lemma 3.5 to hold, in terms of processes $\left\{\tilde{\boldsymbol{\sigma}}_{i, t}\right\}$ whose spin-flipping is independent of the connections. The rest of the sections then prove that the condition of Lemma 4.6 must be satisfied.

\subsection{Regularity of the Connections: Proof of Lemma 3.2}

Proof. We decompose $\mathbf{J}_{N}$ into a symmetric matrix and an i.i.d. matrix, i.e. $\mathbf{J}_{N}=N^{-1 / 2} \sqrt{\mathfrak{s}} \hat{\mathbf{J}}_{N}+N^{-1 / 2} \sqrt{1-\mathfrak{s}} \tilde{\mathbf{J}}_{N}+$ $N^{-1 / 2} \mathbf{D}_{N}$. Here $\mathbf{D}_{N}$ is diagonal, $\hat{\mathbf{J}}_{N}$ is symmetric and $\tilde{\mathbf{J}}_{N}$ is neither symmetric nor anti-symmetric. The entries in all three matrices can be taken to be i.i.d of zero mean and unit variance (in the symmetric matrix the entries are i.i.d. apart from the symmetry $J^{j k}=J^{k j}$ ). A union-of-events bound implies that

$$
\begin{aligned}
\varlimsup_{N \rightarrow \infty} N^{-1} \log \mathbb{P}\left(\|\mathbf{J}\|_{N}>3\right) \leq & \max \left\{\varlimsup_{N \rightarrow \infty} N^{-1} \log \mathbb{P}\left(N^{-1 / 2} \sqrt{\mathfrak{s}}\left\|\hat{\mathbf{J}}_{N}\right\|>4 / 3\right),\right. \\
& \left.\varlimsup_{N \rightarrow \infty} N^{-1} \log \mathbb{P}\left(N^{-1 / 2} \sqrt{1-\mathfrak{s}}\left\|\tilde{\mathbf{J}}_{N}\right\|>4 / 3\right), \varlimsup_{N \rightarrow \infty} N^{-1} \log \mathbb{P}\left(N^{-1 / 2}\left\|\mathbf{D}_{N}\right\|>1 / 3\right)\right\} .
\end{aligned}
$$

For the last term, using Lemma 3.1

$$
\varlimsup_{N \rightarrow \infty} N^{-1} \log \mathbb{P}\left(N^{-1 / 2}\left\|\mathbf{D}_{N}\right\|>1 / 3\right)=\varlimsup_{N \rightarrow \infty} \sup _{1 \leq p \leq N} N^{-1} \log \mathbb{P}\left(N^{-1 / 2}\left|D_{N, p p}\right|>1 / 3\right)<0 .
$$

It is a standard result from random matrix theory [3] that

$$
\varlimsup_{N \rightarrow \infty} N^{-1} \log \mathbb{P}\left(N^{-1 / 2} \sqrt{\mathfrak{s}}\left\|\hat{\mathbf{J}}_{N}\right\|>4 / 3\right)<0 .
$$


The last bound follows from recent results on the maximum eigenvalue of the Ginibre ensemble [57] ,

$$
\varlimsup_{N \rightarrow \infty} N^{-1} \log \mathbb{P}\left(N^{-1 / 2} \sqrt{1-\mathfrak{s}}\left\|\tilde{\mathbf{J}}_{N}\right\|>4 / 3\right)<0 .
$$

For (2), it may be observed that

$$
\mathbb{E}^{\hat{\mu}_{t}^{N}}\left[\left(g^{p}\right)^{2}\right]=N^{-1} \sum_{j \in I_{N}}\left(G_{t}^{p, j}\right)^{2} \leq N^{-1}\left\|\mathbf{J}_{N}\right\| \sum_{j \in I_{N}}\left(\sigma_{t}^{p, j}\right)^{2}=\left\|\mathbf{J}_{N}\right\| \leq 3
$$

as long as $\mathcal{J}_{N}$ holds.

\subsection{Approximating Flow $\Psi_{t}$}

This section proves that $\Psi_{t}$ is a good approximation to the flow $\Phi_{t}$. We now prove Lemma 3.6, which implies that the operator $\Psi$ is compact.

Lemma 3.6. There exists a constant $\bar{C}>0$ such that for all $\mu_{[0, T]} \in \hat{\mathcal{W}}_{[0, T]}$, and writing $\eta_{t}=\Psi_{t} \cdot \mu_{[0, T]}$,

$$
\begin{aligned}
\sup _{0 \leq t \leq T} \mathbb{E}^{\eta_{t}}\left[\|\mathbf{x}\|^{2}\right] & \leq \bar{C} \\
d_{W}\left(\eta_{t}, \eta_{u}\right) & \leq \bar{C} \sqrt{t-u} \text { for all } t \geq u .
\end{aligned}
$$

Proof. To implement the Wasserstein distance, we require a common probability space, and it is easiest to use the stochastic process with marginal probability laws given by (43). That is, $\eta_{t} \in \mathcal{P}$ is the marginal law of the solution $\left(\boldsymbol{\alpha}_{t}, \mathbf{z}_{t}\right)$ of the following stochastic hybrid system. Let $\left\{\tilde{Y}^{p}(t)\right\}_{p \in I_{M}}$ be independent Poisson Counting Processes, and $\left\{\tilde{W}_{t}^{p}\right\}_{p \in I_{M}}$ independent Wiener Processes (these processses are also independent of the Poisson processes $\left\{Y^{p, j}(t)\right\}_{p \in I_{M}, j \in I_{N}}$ and connections $\left\{J^{j k}\right\}_{j, k \in I_{N}}$ used to define the original system) and define for $p \in I_{M}$,

$$
\begin{aligned}
& \alpha_{t}^{p}=\alpha_{0}^{p} A \cdot \tilde{Y}^{p}\left(\int_{0}^{t} c\left(\alpha_{s}^{p}, x_{s}^{p}\right) d s\right) \\
& \mathbf{x}_{t}=\mathbf{x}_{0}+\int_{0}^{t} \mathbf{m}^{\mu_{s}}\left(\boldsymbol{\alpha}_{s}, \mathbf{x}_{s}\right) d s+\int_{0}^{t} \mathbf{D}^{\mu_{s}} d \tilde{\mathbf{W}}_{s}, \text { where } D_{i j}^{\mu_{s}}=2 \sqrt{L_{i i}^{\mu_{s}}} \delta(i, j),
\end{aligned}
$$

and the initial random variables $\left(\boldsymbol{\alpha}_{0}, \mathbf{x}_{0}\right)$ are distributed according to $\mu_{0}$. One easily checks that a unique solution exists to the above equation.

We first establish that there exists a constant $\tilde{C}$ such that

$$
\frac{d}{d t} \mathbb{E}^{\eta_{t}}\left[\|\mathbf{x}\|^{2}\right] \leq \tilde{C}\left\{1+\mathbb{E}^{\eta_{t}}\left[\|\mathbf{x}\|^{2}\right]\right\} .
$$

Thanks to Ito's Lemma,

$$
\left\|\mathbf{x}_{t}\right\|^{2}=\left\|\mathbf{x}_{0}\right\|^{2}+\int_{0}^{t}\left\{\sum_{p \in I_{M}} 4 L_{p p}^{\mu_{s}}+2\left\langle\mathbf{x}_{s}, \mathbf{m}^{\mu_{s}}\left(\boldsymbol{\alpha}_{s}, \mathbf{x}_{s}\right)\right\rangle\right\} d s+2 \int_{0}^{t}\left\langle\mathbf{x}_{s}, \mathbf{D}^{\mu_{s}} d \tilde{\mathbf{W}}_{s}\right\rangle,
$$

where $D_{i j}^{\mu_{s}}=2 \sqrt{L_{i i}^{\mu_{s}}} \delta(i, j)$. It follows from (36) (and the Cauchy-Schwarz Inequality) that

$$
\left\langle\mathbf{x}_{s}, \mathbf{m}^{\mu_{s}}\left(\boldsymbol{\alpha}_{s}, \mathbf{x}_{s}\right)\right\rangle \leq C\left\|\mathbf{x}_{s}\right\|^{2}+C\left\|\mathbf{x}_{s}\right\|\left(1+\sup _{s \in[0, T]} \mathbb{E}^{\mu_{s}}\left[\|\mathbf{x}\|^{2}\right]^{1 / 2}\right)
$$

The definition of $\hat{\mathcal{W}}_{[0, T]}$ implies that $\sup _{s \in[0, T]} \mathbb{E}^{\mu_{s}}\left[\|\mathbf{g}\|^{2}\right] \leq 3$, and it is immediate from the definition that $\left|L_{i i}^{\mu_{s}}\right| \leq c_{1}$. Thus taking expectations of both sides of (63), we obtain (62) as required. 
An application of Gronwall's Inequality to (62) implies that

$$
\sup _{0 \leq t \leq T} \mathbb{E}^{\eta_{t}}\left[\|\mathbf{x}\|^{2}\right] \leq\left(\tilde{C} T+\mathbb{E}^{\mu_{0}}\left[\|\mathbf{x}\|^{2}\right]\right) \exp (\tilde{C} T)
$$

which establishes the first identity, since (by definition) $\mathbb{E}^{\mu_{0}}\left[\|\mathbf{x}\|^{2}\right] \leq 3$. It remains to demonstrate uniform continuity. It follows from Ito's Lemma that for all $t>u$,

$$
\left\|\mathbf{x}_{t}-\mathbf{x}_{u}\right\|^{2}=2 \int_{u}^{t}\left\langle\mathbf{x}_{s}-\mathbf{x}_{u}, \mathbf{m}^{\mu_{s}}\left(\boldsymbol{\alpha}_{s}, \mathbf{x}_{s}\right)\right\rangle d s+4 \int_{u}^{t} \sum_{i \in I_{M}} L_{i i}^{\mu_{s}} d s+2 \int_{u}^{t}\left\langle\mathbf{x}_{s}-\mathbf{x}_{u}, \mathbf{D}^{\mu_{s}} d \mathbf{W}_{s}\right\rangle .
$$

We thus find that, using the Cauchy-Schwarz inequality,

$$
\frac{d}{d t} \mathbb{E}\left[\left\|\mathbf{x}_{t}-\mathbf{x}_{u}\right\|^{2}\right] \leq 2 \mathbb{E}\left[\left\|\mathbf{x}_{t}-\mathbf{x}_{u}\right\|^{2}\right]^{1 / 2} \mathbb{E}\left[\left\|\mathbf{m}^{\mu_{t}}\left(\boldsymbol{\alpha}_{t}, \mathbf{x}_{t}\right)\right\|^{2}\right]^{1 / 2}+2 M c_{1},
$$

since $\left|L_{i i}^{\mu_{t}}\right|$ is uniformly upperbounded by $c_{1}$ (the uniform upperbound for the jump intensity). It follows from (36) that, using the inequality $(a+b)^{2} \leq 2 a^{2}+2 b^{2}$,

$$
\mathbb{E}\left[\left\|\mathbf{m}^{\mu_{t}}\left(\boldsymbol{\alpha}_{t}, \mathbf{x}_{t}\right)\right\|^{2}\right] \leq 2 C^{2} \mathbb{E}\left[\left\|\mathbf{x}_{t}\right\|^{2}\right]+2 C^{2}\left\{1+\mathbb{E}^{\mu_{t}}\left[\|\mathbf{x}\|^{2}\right]^{1 / 2}\right\}^{2}
$$

Thanks to the definition of $\hat{\mathcal{W}}_{[0, T]}, \mathbb{E}^{\mu_{t}}\left[\|\mathbf{x}\|^{2}\right] \leq 3$. It therefore follows from (64) that there exists a constant $\hat{C}$ such that

$$
\begin{aligned}
\frac{d}{d t} \mathbb{E}\left[\left\|\mathbf{x}_{t}-\mathbf{x}_{u}\right\|^{2}\right] & \leq \hat{C} \mathbb{E}\left[\left\|\mathbf{x}_{t}-\mathbf{x}_{u}\right\|^{2}\right]^{1 / 2}+2 M c_{1} \\
& \leq \hat{C} \mathbb{E}\left[\left\|\mathbf{x}_{t}-\mathbf{x}_{u}\right\|^{2}\right]+\hat{C}+2 M c_{1} .
\end{aligned}
$$

Gronwall's Inequality now implies that

$$
\mathbb{E}\left[\left\|\mathbf{x}_{t}-\mathbf{x}_{u}\right\|^{2}\right] \leq(t-u) \exp \{(t-u) \hat{C}\}\left(2 M c_{1}+\hat{C}\right),
$$

and Jensen's Inequality therefore implies that

$$
\mathbb{E}\left[\left\|\mathbf{x}_{t}-\mathbf{x}_{u}\right\|\right] \leq\left((t-u) \exp \{(t-u) \hat{C}\}\left(2 M c_{1}+\hat{C}\right)\right)^{1 / 2} .
$$

The uniform bound $c_{1}$ for the intensity of the spin-flipping implies that

$$
\mathbb{E}\left[\left\|\boldsymbol{\alpha}_{t}-\boldsymbol{\alpha}_{u}\right\|^{2}\right] \leq 4 M(t-u) c_{1}
$$

The above two identities imply (59).

We now prove Lemma 3.3.

Proof. The second result in Lemma 3.6 implies that all elements of $\Psi \cdot \mathcal{W}_{[0, T]}$ are uniformly continuous. The first result in Lemma 3.6 implies that the individual marginals $\left\{\eta_{t}\right\}$ belong to the compact space of measures

$$
\overline{\mathcal{P}}=\left\{\mu \in \mathcal{P}: \mathbb{E}^{\mu}\left[\|\mathbf{g}\|^{2}\right] \leq \bar{C}\right\} .
$$

(This space is compact thanks to Prokhorov's Theorem). It thus follows from the generalized Arzela-Ascoli Theorem [36] that $\Psi \cdot \mathcal{W}_{[0, T]}$ is compact in $\mathcal{C}([0, T], \mathcal{P})$ (this space being endowed with the supremum metric $(44))$.

Suppose for a contradiction that the lemma were not true. Then there would have to exist some $\tilde{\epsilon}>0$ and some sequence $\mu^{n} \in \hat{\mathcal{W}}_{2}$ such that $\tilde{d}_{T}\left(\Psi \cdot \mu^{n}, \mu^{n}\right)<n^{-1}$ and $\tilde{d}_{T}\left(\Phi \cdot \mu_{0}^{n}, \mu^{n}\right) \geq \tilde{\epsilon}$. The compactness of the space $\Psi \cdot \hat{\mathcal{W}}_{[0, T]}$ means that $\left(\Psi \cdot \mu^{n}\right)_{n \in \mathbb{Z}^{+}}$must have a convergent subsequence $\left(\Psi \cdot \mu^{p_{n}}\right)_{n \in \mathbb{Z}^{+}}$, converging to some $\phi=\left(\phi_{t}\right)_{t \in[0, T]}$. Since $\tilde{d}_{T}\left(\Psi \cdot \mu^{n}, \mu^{n}\right)<n^{-1}$, it must be that $\mu^{p_{n}} \rightarrow \phi$ as well. Since $\Psi$ is continuous, 
$\Psi \cdot \mu^{p_{n}}$ also converges to $\Psi \cdot \phi$. We thus find that $\Psi \cdot \phi=\phi$. This contradicts the uniqueness of the fixed point $\xi_{t}$ established in Lemma 2.2 .

It remains to prove (46). First we note that for small enough $\epsilon$, we are certain to avoid the pathological situation of $\Lambda^{\Phi_{t} \cdot \hat{\mu}^{N}} \rightarrow 0$ for $t \leq \tau_{N}$. This event would imply that $\left\|\left(\mathbf{K}^{\xi_{t}}\right)^{-1}\right\| \rightarrow \infty$ (and the PDE in (31) would no longer be accurate). Let $\epsilon_{\mathfrak{c}}>0$ be the largest number such that

$$
\left\{\mu \in \mathcal{P}: \Lambda^{\mu} \geq \mathfrak{c}\right\}=\left\{\mu \in \mathcal{P}: \Lambda^{\mu} \geq \mathfrak{c} \text { and } \Lambda^{\nu} \geq \mathfrak{c} / 2 \text { for all } \nu \text { such that } d_{W}(\mu, \nu) \leq \epsilon_{\mathfrak{c}}\right\}
$$

Such an $\epsilon_{\mathfrak{c}}$ always exists because the map $\Lambda^{\mu}$ is continuous. We will thus assume (throughout the rest of this paper) that $\epsilon \leq \epsilon_{\mathfrak{c}}$, because in any case if the RHS of the following inequality is less than zero, then the LHS must be less than zero too, i.e.

$$
\begin{aligned}
\varlimsup_{N \rightarrow \infty} N^{-1} \log \mathbb{P}\left(\sup _{t \leq \tau_{N}} d_{W}\left(\Phi_{t} \cdot \hat{\mu}^{N}\left(\boldsymbol{\sigma}_{0}, \mathbf{G}_{0}\right), \hat{\mu}^{N}\left(\boldsymbol{\sigma}_{t}, \mathbf{G}_{t}\right)\right) \geq \epsilon\right) \leq \\
\varlimsup_{N \rightarrow \infty} N^{-1} \log \mathbb{P}\left(\sup _{t \leq \tau_{N}} d_{W}\left(\Phi_{t} \cdot \hat{\mu}^{N}\left(\boldsymbol{\sigma}_{0}, \mathbf{G}_{0}\right), \hat{\mu}^{N}\left(\boldsymbol{\sigma}_{t}, \mathbf{G}_{t}\right)\right) \geq \min \left\{\epsilon, \epsilon_{\mathfrak{c}}\right\}\right) .
\end{aligned}
$$

With this choice of $\epsilon$, we are assured that $\mathbb{P}\left(\mathcal{Q}_{N}^{c}\right)=0$ where

$$
\mathcal{Q}_{N}=\left\{\mathbf{H}^{\Phi_{t} \cdot \hat{\mu}_{0}^{N}}=\left(\mathbf{K}^{\Phi_{t} \cdot \hat{\mu}_{0}^{N}}\right)^{-1} \text { as long as } t<\tau_{N} \text { and } d_{W}\left(\Phi_{t} \cdot \hat{\mu}^{N}\left(\boldsymbol{\sigma}_{0}, \mathbf{G}_{0}\right), \hat{\mu}^{N}\left(\boldsymbol{\sigma}_{t}, \mathbf{G}_{t}\right)\right) \leq \epsilon\right\} .
$$

As long as $\epsilon \leq \epsilon_{\mathfrak{c}}$ (defined just above (71)), and $\delta$ is chosen such that (45) is satisfied, then (46) must hold.

\subsection{Proofs of the Remaining Lemmas}

Lemma 3.7. For any $\epsilon>0$, for all sufficiently large $n$,

$$
\sup _{0 \leq b<n} \varlimsup_{N \rightarrow \infty} N^{-1} \log \mathbb{P}\left(\mathcal{J}_{N} \text { and } \sup _{s \in\left[t_{b}^{(n)}, t_{b+1}^{(n)}\right]} d_{W}\left(\hat{\mu}^{N}\left(\boldsymbol{\sigma}_{b}, \mathbf{G}_{b}\right), \hat{\mu}^{N}\left(\boldsymbol{\sigma}_{s}, \mathbf{G}_{s}\right)\right) \geq \epsilon\right)<0 .
$$

Proof. It follows from the definition that

$$
d_{W}\left(\hat{\mu}^{N}\left(\boldsymbol{\sigma}_{b}, \mathbf{G}_{b}\right), \hat{\mu}^{N}\left(\boldsymbol{\sigma}_{s}, \mathbf{G}_{s}\right)\right) \leq\left(N^{-1} \sum_{j \in I_{N}, i \in I_{M}}\left|G_{b}^{i, j}-G_{s}^{i, j}\right|^{2}\right)^{\frac{1}{2}}+N^{-1} \sum_{j \in I_{N}, i \in I_{M}}\left|\sigma_{s}^{i, j}-\sigma_{b}^{i, j}\right| .
$$

The renewal property of Poisson Processes implies that the following processes $\left\{Y_{a}^{q, j}(t)\right\}_{q \in I_{M}, j \in I_{N}}$ are Poissonian:

$$
Y_{b}^{q, j}(t):=Y^{q, j}\left(t+\int_{0}^{t_{b}^{(n)}} c\left(\sigma_{s}^{q, j}, G_{s}^{q, j}\right) d s\right)-Y^{q, j}\left(\int_{0}^{t_{b}^{(n)}} c\left(\sigma_{s}^{q, j}, G_{s}^{q, j}\right) d s\right)
$$

Now as long as the event $\mathcal{J}_{N}$ holds,

$$
\begin{aligned}
N^{-1} \sum_{j \in I_{N}, i \in I_{M}}\left|G_{b}^{i, j}-G_{s}^{i, j}\right|^{2} & \leq \frac{3}{N} \sum_{j \in I_{N}, i \in I_{M}}\left\{\sigma_{s}^{i, j}-\sigma_{b}^{i, j}\right\}^{2} \\
& \leq 12 N^{-1} \sum_{j \in I_{N}, i \in I_{M}} Y_{b}^{i, j}\left(c_{1}\left\{s-t_{b}^{(n)}\right\}\right) \leq 12 N^{-1} \sum_{j \in I_{N}, i \in I_{M}} Y_{b}^{i, j}\left(c_{1} \Delta\right) .
\end{aligned}
$$

Similarly, $N^{-1} \sum_{j \in I_{N}, i \in I_{M}}\left|\sigma_{s}^{i, j}-\sigma_{b}^{i, j}\right| \leq 2 N^{-1} \sum_{j \in I_{N}, i \in I_{M}} Y_{b}^{i, j}\left(c_{1} \Delta\right)$. Writing $\bar{\epsilon}$ to be such that $\sqrt{12 \bar{\epsilon}}+2 \bar{\epsilon}=\epsilon$ and noting that $Y_{b}^{i, j}$ is non-decreasing, it thus suffices to prove that for any $\bar{\epsilon}>0$,

$$
\varlimsup_{N \rightarrow \infty} N^{-1} \log \mathbb{P}\left(\mathcal{J}_{N} \text { and } N^{-1} \sum_{j \in I_{N}, i \in I_{M}} Y_{b}^{i, j}\left(c_{1} \Delta\right) \geq \bar{\epsilon}\right)<0 .
$$


Taking $\Delta$ to be such that $c_{1} \Delta \leq \bar{\epsilon} / 2$, it suffices to prove that

$$
\varlimsup_{N \rightarrow \infty} N^{-1} \log \mathbb{P}\left(\mathcal{J}_{N} \text { and } N^{-1} \sum_{j \in I_{N}, i \in I_{M}} Y_{b}^{i, j}\left(c_{1} \Delta\right)-c_{1} \Delta M \geq \bar{\epsilon} / 2\right)<0 .
$$

Since the $\left\{Y^{i, j}\right\}_{i \in I_{M}, j \in I_{N}}$ are independent, and $\mathbb{E}\left[N^{-1} \sum_{j \in I_{N}, i \in I_{M}} Y_{b}^{i, j}\left(c_{1} \Delta\right)\right]=M c_{1} \Delta$, Sanov's Theorem implies (76) [25].

We now prove Lemma 3.4.

Proof. Let $\eta_{b} \in \mathcal{P}$ be the law of the same stochastic process as $\xi_{b}(\boldsymbol{\sigma}, \mathbf{G})$, except that the law of the initial value at time $t_{b}^{(n)}$ is given by $\Psi_{b} \cdot \hat{\mu}^{N}$ rather than the empirical measure. More precisely, writing $\Psi_{b} \cdot \hat{\mu}^{N}$ to be the law of random variables $\left(\boldsymbol{\alpha}_{b}, \mathbf{x}_{b}\right)$, define $\eta_{b}$ to be the law of $\left(\boldsymbol{\beta}_{\Delta+t_{b}^{(n)}}, \mathbf{z}_{\Delta+t_{b}^{(n)}}\right)$, where, writing $A \cdot x=(-1)^{x}$, for $p \in I_{M}$, for $t \geq t_{b}^{(n)}$,

$$
\begin{aligned}
& \beta_{t}^{p}=\alpha_{b}^{p} A \cdot \tilde{Y}^{p}\left(\left(t-t_{b}^{(n)}\right) c\left(\alpha_{b}^{p}, x_{b}^{p}\right)\right) \\
& \mathbf{z}_{t}=\mathbf{x}_{b}+\left(t-t_{b}^{(n)}\right) \mathbf{m}^{\hat{\mu}_{b}^{N}}\left(\boldsymbol{\alpha}_{b}, \mathbf{x}_{b}\right)+\mathbf{D}^{\hat{\mu}_{b}^{N}} \tilde{\mathbf{W}}_{t-t_{b}^{(n)}}, \text { where } D_{i j}^{\hat{\mu}_{b}^{N}}=2 \sqrt{L_{i i}^{\hat{\mu}_{b}^{N}}} \delta(i, j) .
\end{aligned}
$$

Thanks to the fact that $\exp \left(\mathfrak{u} t_{b}^{(n)} / T+\mathfrak{u} \Delta /(2 T)-\mathfrak{u}\right) \geq \exp \left(\mathfrak{u} t_{b}^{(n)} / T+\mathfrak{u} \Delta / 4 T-\mathfrak{u}\right)+\exp \left(\mathfrak{u} t_{b}^{(n)} / T-\mathfrak{u}\right) \mathfrak{u} \Delta / 4 T$, analogously to (55) we find that

$$
\begin{array}{r}
\left\{d_{W}\left(\Psi_{b+1} \cdot \hat{\mu}^{N}, \xi_{b}\right)>\tilde{\epsilon} \exp \left(\mathfrak{u} t_{b}^{(n)} / T+\mathfrak{u} \Delta / 2 T-\mathfrak{u}\right)\right\} \subseteq\left\{d_{W}\left(\eta_{b}, \xi_{b}\right)>\tilde{\epsilon} \exp \left(\mathfrak{u} t_{b}^{(n)} / T+\mathfrak{u} \Delta / 4 T-\mathfrak{u}\right)\right\} \\
\cup\left\{d_{W}\left(\Psi_{b+1} \cdot \hat{\mu}^{N}, \eta_{b}\right)>\exp \left(\mathfrak{u} t_{b}^{(n)} / T+\mathfrak{u} \Delta / 4 T-\mathfrak{u}\right) \tilde{\epsilon} \mathfrak{u} \Delta / 4 T\right\}
\end{array}
$$

Thanks to Lemma 3.1, it thus suffices for us to prove the following three inequalities,

$$
\begin{aligned}
\varlimsup_{N \rightarrow \infty} N^{-1} \log \mathbb{P}\left(\mathcal{J}_{N} \text { and } d_{W}\left(\eta_{b}, \xi_{b}\right)>\tilde{\epsilon} \exp \left(\mathfrak{u} t_{b}^{(n)} / T+\mathfrak{u} \Delta / 4 T-\mathfrak{u}\right)\right. \\
\left.\quad \text { and } d_{W}\left(\Psi_{b} \cdot \hat{\mu}^{N}, \hat{\mu}_{b}^{N}\right) \leq \tilde{\epsilon} \exp \left(\mathfrak{u} t_{b}^{(n)} / T-\mathfrak{u}\right)\right)<0 \text { and }
\end{aligned}
$$

for some $\epsilon_{0}>0$,

$$
\begin{aligned}
& \varlimsup_{N \rightarrow \infty} N^{-1} \log \mathbb{P}\left(\mathcal{J}_{N}, \sup _{s \in\left[t_{b}^{(n)}, t_{b+1}^{(n)}\right]} d_{W}\left(\hat{\mu}_{s}^{N}, \hat{\mu}_{b}^{N}\right)>\epsilon_{0}\right)<0 \\
& \varlimsup_{N \rightarrow \infty} N^{-1} \log \mathbb{P}\left(\mathcal{J}_{N}, \sup _{s \in\left[t_{b}^{(n)}, t_{b+1}^{(n)}\right]} d_{W}\left(\hat{\mu}_{s}^{N}, \hat{\mu}_{b}^{N}\right) \leq \epsilon_{0}, d_{W}\left(\eta_{b}, \Psi_{b+1} \cdot \hat{\mu}^{N}\right)>\frac{\tilde{\epsilon} \mathfrak{u} \Delta}{4 T} \exp \left(\mathfrak{u} t_{b}^{(n)} / T+\mathfrak{u} \Delta / 4 T-\mathfrak{u}\right)\right)<0 .
\end{aligned}
$$

It has already been proved in Lemma 3.7 that for any $\epsilon_{0}$, for all large enough $n$ (82) must hold.

Proof of (83).

We compare the stochastic processes (60)-(61) whose law is $\Psi_{b+1} \cdot \hat{\mu}^{N}$ to the stochastic processes (78)-(79) whose law is $\eta_{b}$. Notice that these processes have the same initial condition at time $t_{b}^{(n)}$. Using Ito's Lemma, for $t \geq t_{b}^{(n)}$,

$$
\begin{aligned}
\left\|\mathbf{x}_{t}-\mathbf{z}_{t}\right\|^{2}=\int_{t_{b}^{(n)}}^{t}\left\{2\left\langle\mathbf{x}_{s}-\mathbf{z}_{s}, \mathbf{m}^{\hat{\mu}_{s}^{N}}\left(\boldsymbol{\alpha}_{s}, \mathbf{x}_{s}\right)-\mathbf{m}^{\hat{\mu}_{b}^{N}}\left(\boldsymbol{\alpha}_{b}, \mathbf{x}_{b}\right)\right\rangle+4\right. & \left.\sum_{p \in I_{M}}\left(D_{p p}^{\hat{\mu}_{s}^{N}}-D_{p p}^{\hat{\mu}_{b}^{N}}\right)^{2}\right\} d s \\
& +2 \int_{t_{b}^{(n)}}^{t}\left\langle\mathbf{x}_{s}-\mathbf{z}_{s},\left(\mathbf{D}^{\hat{\mu}_{s}^{N}}-\mathbf{D}^{\hat{\mu}_{b}^{N}}\right) d \tilde{\mathbf{W}}_{s}\right\rangle
\end{aligned}
$$


Analogously to the bound in (64), one easily establishes the following uniform bound for the moments

$$
\sup _{t \in\left[t_{b}^{(n)}, t_{b+1}^{(n)}\right]}\left\{\left\|\mathbf{x}_{t}\right\|^{2},\left\|\mathbf{z}_{t}\right\|^{2}\right\} \leq \breve{C}
$$

for some constant $\breve{C}$. Using the Lipschitz inequality for $\mathbf{m}$ in Lemma 2.6, and making use of the uniform bound in (85), there exists a constant $\bar{C}$ such that for all $s \in\left[t_{b}^{(n)}, t_{b+1}^{(n)}\right]$,

$$
\left\|\mathbf{m}^{\hat{\mu}_{s}^{N}}\left(\boldsymbol{\alpha}_{s}, \mathbf{x}_{s}\right)-\mathbf{m}^{\hat{\mu}_{b}^{N}}\left(\boldsymbol{\alpha}_{b}, \mathbf{x}_{b}\right)\right\| \leq \bar{C}\left(\left\|\mathbf{x}_{s}-\mathbf{x}_{b}\right\|+\left\|\boldsymbol{\alpha}_{s}-\boldsymbol{\alpha}_{b}\right\|\right)
$$

Taking expectations of both sides of (84), employing the Cauchy-Schwarz Inequality, and assuming that $\sup _{s \in\left[t_{b}^{(n)}, t_{b+1}^{(n)}\right]} d_{W}\left(\hat{\mu}_{s}^{N}, \hat{\mu}_{b}^{N}\right) \leq \epsilon_{0}$, we obtain that

$$
\mathbb{E}\left[\left\|\mathbf{x}_{t}-\mathbf{z}_{t}\right\|^{2}\right] \leq 2 \grave{C} \int_{t_{b}^{(n)}}^{t}\left(\mathbb{E}\left[\left\|\mathbf{x}_{s}-\mathbf{z}_{s}\right\|^{2}\right]+\mathbb{E}\left[\left\|\mathbf{x}_{s}-\mathbf{z}_{s}\right\|^{2}\right]^{1 / 2} \mathbb{E}\left[\left\|\boldsymbol{\alpha}_{s}-\boldsymbol{\alpha}_{b}\right\|^{2}\right]^{1 / 2}\right) d s+4\left(t-t_{b}^{(n)}\right) \epsilon_{0} .
$$

Properties of the Poisson Process (see for example Lemma 8.1) dictate that $\mathbb{E}\left[\left\|\boldsymbol{\alpha}_{s}-\boldsymbol{\alpha}_{b}\right\|^{2}\right] \leq 4 c_{1} \Delta$, as long as $s-t_{b}^{(n)} \leq \Delta$. Thus for all $t$ such that $\mathbb{E}\left[\left\|\mathbf{x}_{t}-\mathbf{z}_{t}\right\|^{2}\right] \leq \Delta$, it must hold that

$$
\mathbb{E}\left[\left\|\mathbf{x}_{t}-\mathbf{z}_{t}\right\|^{2}\right] \leq 2 \grave{C} \int_{t_{b}^{(n)}}^{t}\left(\mathbb{E}\left[\left\|\mathbf{x}_{s}-\mathbf{z}_{s}\right\|^{2}\right] d s+\left(t-t_{b}^{(n)}\right)\left\{\epsilon_{0}+4 \Delta \grave{C} \sqrt{c_{1}}\right\} .\right.
$$

We thus find from Gronwall's Inequality that for any $\bar{\epsilon}>0$, through choosing $\epsilon_{0}$ to be sufficiently small, and $n$ to be sufficiently large,

$$
\sup _{t \in\left[t_{b}^{(n)}, t_{b+1}^{(n)}\right]} \mathbb{E}\left[\left\|\mathbf{x}_{t}-\mathbf{z}_{t}\right\|^{2}\right] \leq \Delta \bar{\epsilon}
$$

Using the compensated Poisson Process representation, we obtain that

$$
\begin{aligned}
\mathbb{E}\left[\left\|\boldsymbol{\alpha}_{t}-\boldsymbol{\beta}_{t}\right\|^{2}\right] & \leq 4 \sum_{p \in I_{M}} \mathbb{E}\left[\mid \tilde{Y}^{p}\left(\left(t-t_{b}^{(n)}\right) c\left(\alpha_{b}^{p}, z_{b}^{p}\right)-\tilde{Y}^{p}\left(\int_{t_{b}^{(n)}}^{t} c\left(\alpha_{s}^{p}, x_{s}^{p}\right) d s\right) \mid\right]\right. \\
& \leq 4 \sum_{p \in I_{M}} \mathbb{E}\left[\int_{t_{b}^{(n)}}^{t}\left|c\left(\alpha_{b}^{p}, z_{b}^{p}\right)-c\left(\alpha_{s}^{p}, x_{s}^{p}\right)\right| d s\right] \\
& \leq 4 M\left(c_{1}+c_{L}\right) \Delta \sup _{s \in\left[t_{b}^{(n)}, t_{b+1}^{(n)}\right]} \mathbb{E}\left[\left\|\mathbf{x}_{s}-\mathbf{x}_{b}\right\|+\left\|\boldsymbol{\alpha}_{s}-\boldsymbol{\alpha}_{b}\right\|\right],
\end{aligned}
$$

using the fact that $c(\cdot, \cdot)$ is Lipschitz and bounded. Since the expectation in the last term goes to zero as $\Delta \rightarrow 0$, it follows from (87) and (88) that for sufficiently large $\Delta$,

$$
d_{W}\left(\eta_{b}, \Psi_{b+1} \cdot \hat{\mu}^{N}\right) \leq \frac{\tilde{\epsilon} \mathfrak{u} \Delta}{4 T} \exp \left(\mathfrak{u} t_{b}^{(n)} / T+\mathfrak{u} \Delta / 4 T-\mathfrak{u}\right) .
$$

We have thus established (83) and it remains to prove (81). Suppose that $d_{W}\left(\Psi_{b} \cdot \hat{\mu}^{N}, \hat{\mu}_{b}^{N}\right) \leq \tilde{\epsilon} \exp \left(\mathfrak{u} t_{b}^{(n)} / T-\right.$ $\mathfrak{u})$. The definition of the Wasserstein distance implies that for any $\delta>0$, there must exist a common probability space supporting the random variables $(\boldsymbol{\zeta}, \mathbf{x}, \boldsymbol{\beta}, \mathbf{z})$, with $\hat{\mu}_{b}^{N}$ the law of $(\boldsymbol{\zeta}, \mathbf{x})$, and $\Psi_{b} \cdot \hat{\mu}^{N}$ the law of $(\boldsymbol{\beta}, \mathbf{z})$, and such that

$$
\mathbb{E}[\|\boldsymbol{\zeta}-\boldsymbol{\beta}\|+\|\mathbf{x}-\mathbf{z}\|] \leq \delta+\tilde{\epsilon} \exp \left(\mathfrak{u} t_{b}^{(n)} / T-\mathfrak{u}\right) .
$$

We append the mutually independent Poisson processes $\left\{\tilde{Y}^{p}(t)\right\}_{p \in I_{M}}$ and Brownian motions $\left\{\tilde{W}_{t}^{p}\right\}_{p \in I_{M}}$ to this same space, and define $\left(\boldsymbol{\zeta}_{\Delta}, \mathbf{x}_{\Delta}\right)$ to satisfy (53) -(54) and $\left(\boldsymbol{\beta}_{\Delta}, \mathbf{z}_{\Delta}\right)$ to satisfy (78)-(79). We then observe using the triangle inequality that

$$
\begin{aligned}
\mathbb{E}\left[\left\|\boldsymbol{\zeta}_{\Delta}-\boldsymbol{\beta}_{\Delta}\right\|+\left\|\mathbf{x}_{\Delta}-\mathbf{z}_{\Delta}\right\|\right] & \leq \mathbb{E}\left[\|\boldsymbol{\zeta}-\boldsymbol{\beta}\|+\|\mathbf{x}-\mathbf{z}\|+\left\|\boldsymbol{\zeta}_{\Delta}-\boldsymbol{\zeta}+\boldsymbol{\beta}-\boldsymbol{\beta}_{\Delta}\right\|+\left\|\mathbf{x}_{\Delta}-\mathbf{x}+\mathbf{z}-\mathbf{z}_{\Delta}\right\|\right] \\
& \leq \delta+\tilde{\epsilon} \exp \left(\mathfrak{u} t_{b}^{(n)} / T-\mathfrak{u}\right)+\mathbb{E}\left[\left\|\boldsymbol{\zeta}_{\Delta}-\boldsymbol{\zeta}+\boldsymbol{\beta}-\boldsymbol{\beta}_{\Delta}\right\|+\left\|\mathbf{x}_{\Delta}-\mathbf{x}+\mathbf{z}-\mathbf{z}_{\Delta}\right\|\right] \text { and }
\end{aligned}
$$




$$
\mathbb{E}\left[\left\|\boldsymbol{\zeta}_{\Delta}-\boldsymbol{\zeta}+\boldsymbol{\beta}-\boldsymbol{\beta}_{\Delta}\right\| \mid \boldsymbol{\zeta}, \boldsymbol{\beta}, \mathbf{x}, \mathbf{g}\right] \leq 2 \sum_{p \in I_{M}} \mathbb{E}\left[\left|\tilde{Y}^{p}\left(\Delta c\left(\zeta^{p}, x^{p}\right)\right)-\tilde{Y}^{p}\left(\Delta c\left(\beta^{p}, z^{p}\right)\right)\right| \mid \boldsymbol{\zeta}, \boldsymbol{\beta}, \mathbf{x}, \mathbf{g}\right]
$$

Define $v_{p}=\inf \left\{c\left(\beta^{p}, z^{p}\right), c\left(\zeta^{p}, x^{p}\right)\right\}$ and let $\left\{\breve{Y}^{p}, \hat{Y}^{p}, \grave{Y}^{p}\right\}_{p \in I_{M}}$ be independent Poisson Processes. Using the additive property of Poisson Processes [27], we have the following representation

$$
\begin{gathered}
\tilde{Y}^{p}\left(\Delta c\left(\zeta^{p}, x^{p}\right)\right)=\breve{Y}^{p}\left(v_{p}\right)+\hat{Y}^{p}\left(\Delta\left[c\left(\zeta^{p}, x^{p}\right)-v_{p}\right]_{+}\right) \\
\tilde{Y}^{p}\left(\Delta c\left(\beta^{p}, z^{p}\right)\right)=\breve{Y}^{p}\left(v_{p}\right)+\grave{Y}^{p}\left(\Delta\left[c\left(\beta^{p}, z^{p}\right)-v_{p}\right]_{+}\right) .
\end{gathered}
$$

Hence (91) implies that

$$
\begin{aligned}
\mathbb{E}\left[\left\|\boldsymbol{\zeta}_{\Delta}-\boldsymbol{\zeta}+\boldsymbol{\beta}-\boldsymbol{\beta}_{\Delta}\right\| \mid \boldsymbol{\zeta}, \boldsymbol{\beta}, \mathbf{x}, \mathbf{g}\right] & \leq 2 \sum_{p \in I_{M}} \mathbb{E}\left[\hat{Y}^{p}\left(\Delta\left[c\left(\zeta^{p}, x^{p}\right)-v_{p}\right]_{+}\right)+\grave{Y}^{p}\left(\Delta\left[c\left(\beta^{p}, z^{p}\right)-v_{p}\right]_{+}\right) \mid \boldsymbol{\zeta}, \boldsymbol{\beta}, \mathbf{x}, \mathbf{g}\right] \\
& =2 \Delta \sum_{p \in I_{M}}\left|c\left(\zeta^{p}, x^{p}\right)-c\left(\beta^{p}, z^{p}\right)\right| \\
& \leq 2 M \Delta \sup _{p \in I_{M}}\left\{c_{1}\left|\zeta^{p}-\beta^{p}\right|+c_{L}\left|x^{p}-g^{p}\right|\right\}
\end{aligned}
$$

where $c_{1}$ is the uniform upperbound for the jump rate, and $c_{L}$ is the Lipschitz constant for $c$. Taking expectations of both sides, one finds that there exists a constant $\bar{C}>0$ such that

$$
\mathbb{E}\left[\left\|\boldsymbol{\zeta}_{\Delta}-\boldsymbol{\zeta}+\boldsymbol{\beta}-\boldsymbol{\beta}_{\Delta}\right\|\right] \leq \bar{C} \Delta \mathbb{E}[\|\boldsymbol{\zeta}-\boldsymbol{\beta}\|+\|\mathbf{x}-\mathbf{z}\|] .
$$

We analogously find that for a constant $C>0$,

$$
\mathbb{E}\left[\left\|\mathbf{x}_{\Delta}-\mathbf{x}+\mathbf{z}-\mathbf{z}_{\Delta}\right\|\right] \leq C \Delta \mathbb{E}[\|\boldsymbol{\zeta}-\boldsymbol{\beta}\|+\|\mathbf{x}-\mathbf{z}\|],
$$

since the coefficients $\mathbf{m}$ and $\mathbf{L}$ are Lipschitz, as noted in Lemma 2.6. The above results (89)-(93) imply that there exists a constant $\hat{C}>0$ such that

$$
d_{W}\left(\eta_{b}, \xi_{b}\right) \leq d_{W}\left(\hat{\mu}_{b}^{N}, \Psi_{b} \cdot \hat{\mu}^{N}\right)\{1+\hat{C} \Delta\}
$$

Thus as long as $\mathfrak{u} / 4 T>\hat{C}$, if $d_{W}\left(\hat{\mu}_{b}^{N}, \Psi_{b} \cdot \hat{\mu}^{N}\right) \leq \tilde{\epsilon} \exp \left(\mathfrak{u} t_{b}^{(n)} / T-\mathfrak{u}\right)$, it must be that $d_{W}\left(\eta_{b}, \xi_{b}\right) \leq \tilde{\epsilon} \exp \left(\mathfrak{u} t_{b}^{(n)} / T+\right.$ $\mathfrak{u} \Delta / 4 T-\mathfrak{u}$ ), which establishes (81).

\section{Change of Measure}

It remains for us to prove Lemma 3.5. To do this, we must 'separate' the effects of the stochasticity and the disorder on the dynamics by defining new processes $\tilde{\boldsymbol{\sigma}}_{i, t}$ (with $i$ belonging to an index set that grows polynomially in $N$ ) that are such that the spin-flipping is independent of the connections. However it will be seen that $\tilde{\boldsymbol{\sigma}}_{i, t}$ is an excellent approximation to the old process, as long as the empirical process lies in a small subset $\mathcal{V}_{i}^{N}$ of $\mathcal{M}_{1}^{+}\left(\mathcal{D}\left([0, T], \mathcal{E}^{M} \times \mathbb{R}^{M}\right)\right)$. The number of such subsets $\left\{\mathcal{V}_{i}^{N}\right\}$ is polynomial in $N$ : this polynomial growth will be dominated by the exponential decay of the probability bounds of subsequent sections. The fact that the new processes are independent of the connections will allow us to use a conditional Gaussian measure to accurately infer the evolution of the fields over a small time step (in Section 7). In order that we may employ Girsanov's Theorem, it is essential that the processes $\tilde{\boldsymbol{\sigma}}_{i, t}$ are adapted to the filtration $\mathcal{F}_{t}$ as well. The main result of this section is Lemma 4.6: this lemma gives a sufficient condition in terms of the new processes $\tilde{\boldsymbol{\sigma}}_{i, t}$ for the condition of Lemma 3.5 to be satisfied. 


\subsection{Partition of the Probability Space}

Define the pathwise empirical measure

$$
\tilde{\mu}^{N}=N^{-1} \sum_{j \in I_{N}} \delta_{\left(\boldsymbol{\sigma}^{j}, \mathbf{G}^{j}\right)} \in \mathcal{M}_{1}^{+}\left(\mathcal{D}\left([0, T], \mathcal{E}^{M} \times \mathbb{R}^{M}\right)\right) .
$$

The pathwise empirical measure will be used to partition the probability space. Before we partition $\mathcal{M}_{1}^{+}\left(\mathcal{D}\left([0, T], \mathcal{E}^{M} \times \mathbb{R}^{M}\right)\right)$, we must first partition the underlying state space $\mathcal{E}^{M} \times \mathbb{R}^{M}$. For some positive integer $\mathfrak{n}$, define the sets $\left\{D_{i}\right\}_{0 \leq i \leq \mathfrak{n}^{2}+1} \subset \mathbb{R}$ as follows.

$$
\begin{aligned}
D_{0} & =(-\infty,-\mathfrak{n}], \quad D_{\mathfrak{n}^{2}+1}=(\mathfrak{n}, \infty) \\
D_{i} & =\left(-\mathfrak{n}+2(i-1) \mathfrak{n}^{-1},-\mathfrak{n}+2 i \mathfrak{n}^{-1}\right] \text { for } 1 \leq i \leq \mathfrak{n}^{2} .
\end{aligned}
$$

Next, let $\left\{\tilde{D}_{i}\right\}_{1 \leq i \leq C_{\mathfrak{n}}} \subset \mathbb{R}^{M}$ be such that for each $i$,

$$
\tilde{D}_{i}=D_{p_{1}^{i}} \times D_{p_{2}^{i}} \times \ldots D_{p_{M}^{i}},
$$

for integers $\left\{p_{j}^{i}\right\}$. The sets are defined to be such that

$$
\mathbb{R}^{M}=\bigcup_{i=0}^{C_{\mathfrak{n}}} \tilde{D}_{i} \text { and } \tilde{D}_{i} \cap \tilde{D}_{j}=\varnothing \text { if } i \neq j
$$

Next we partition the path space

$$
\mathcal{D}\left([0, T], \mathcal{E}^{M} \times \mathbb{R}^{M}\right)=\bigcup_{i=1}^{\hat{C}_{\mathfrak{n}}} \hat{D}_{i},
$$

where $\left\{\hat{D}_{i}\right\}$ are defined as follows. In constructing this partition, we require a more refined partition of the time interval $[0, T]$ into $(m+1)$ time points $\left\{t_{a}^{(m)}\right\}_{0 \leq a \leq m}$ : this is necessary for us to be able to control the Girsanov Exponent in the next section. It is assumed that $m$ is an integer multiple of $n$ (the integer dictating the number of time points in the previous section). Throughout this section, unless specified otherwise, for $0 \leq a \leq m$, we write $\boldsymbol{\sigma}_{a}:=\boldsymbol{\sigma}_{t_{a}^{(m)}}$. Each $\hat{D}_{i} \subset \mathcal{D}\left([0, T], \mathcal{E}^{M} \times \mathbb{R}^{M}\right)$ is nonempty, and of the form

$$
\hat{D}_{i}=\left\{\boldsymbol{\alpha}_{[0, T]} \times \mathbf{g}_{[0, T]}: \mathbf{g}_{a} \in \tilde{D}_{r_{a}^{i}} \text { and } \boldsymbol{\alpha}_{a} \in \tilde{D}_{q_{a}^{i}} \text { for each } 0 \leq a \leq m\right\},
$$

for indices $\left\{q_{a}^{i}, r_{a}^{i}\right\}_{0 \leq a \leq m}, 1 \leq q_{a}, r_{a} \leq C_{\mathfrak{n}}$. The indices are chosen such that (i) $\hat{D}_{i} \cap \hat{D}_{j}=\varnothing$ if $i \neq j$, (ii) $\hat{D}_{i} \neq \varnothing$ and (iii) (100) is satisfied. Let

$$
\hat{\mathcal{W}}_{2}=\left\{\mu \in \mathcal{M}_{1}^{+}\left(\mathcal{D}\left([0, T], \mathcal{E}^{M} \times \mathbb{R}^{M}\right)\right): \sup _{p \in I_{M}} \sup _{t \in[0, T]} \mathbb{E}^{\mu}\left[\left(g_{t}^{p}\right)^{2}\right] \leq 3\right\} .
$$

Next, for a positive integer $C_{\mathfrak{n}}^{N}$, make the partition

$$
\tilde{\mathcal{W}}_{2}=\bigcup_{i=1}^{C_{\mathrm{n}}^{N}} \mathcal{V}_{i}^{N}
$$

where each $\mathcal{V}_{i}^{N}$ is such that $\mu \in \mathcal{V}_{i}^{N}$ if and only if (i) $\mu \in \hat{\mathcal{W}}_{2}$ and (ii) for all $1 \leq q, r \leq \hat{C}_{\mathfrak{n}}$,

$$
\begin{gathered}
\mu\left(\boldsymbol{\sigma} \in \hat{D}_{q} \text { and } \mathbf{g} \in \hat{D}_{r}\right) \in\left[\hat{u}_{i, q r}^{N}-1 /(2 N), \hat{u}_{i, q r}^{N}+1 /(2 N)\right) \text { for numbers } \\
\hat{u}_{i, q r}^{N} \in\left\{0, N^{-1}, 2 N^{-1}, \ldots, 1-N^{-1}, 1\right\} .
\end{gathered}
$$

It is assumed that the indices are chosen such that (i) $\mathcal{V}_{i}^{N} \neq \varnothing$ and (ii) the partition is disjoint, i.e. $\mathcal{V}_{i}^{N} \cap \mathcal{V}_{j}^{N}=\varnothing$ if $i \neq j$. The motivation for the scaling of $N^{-1}$ for the mass of each set in (104) is that if $\tilde{\mu}^{N} \in \mathcal{V}_{j}^{N}$, then we will know the precise mass assigned to each set, since the empirical process can only assign a mass that is an integer multiple of $N^{-1}$ to each set.

We next prove that the radius of the sets in the partition goes to zero uniformly, in the following sense. 
Lemma 4.1. Define

$$
\mathfrak{U}=\left\{f: \mathcal{E}^{M(m+1)} \times \mathbb{R}^{M(m+1)} \rightarrow \mathbb{R} ;|f(\boldsymbol{\alpha}, \mathbf{x})-f(\boldsymbol{\beta}, \mathbf{g})| \leq \sum_{q \in I_{M}} \sum_{a=0}^{m}\left\{\left|\alpha_{a}^{q}-\beta_{a}^{q}\right|+\left|x_{a}^{q}-g_{a}^{q}\right|\right\} \text { and } f(\cdot, \mathbf{0})=0\right\} .
$$

For $f \in \mathfrak{U}$, write $\hat{f}: \mathcal{D}\left([0, T], \mathcal{E}^{M} \times \mathbb{R}^{M}\right) \rightarrow \mathbb{R}$ to be $\hat{f}(\boldsymbol{\alpha}, \mathbf{x}):=f\left(\left(\alpha_{t_{a}^{(m)}}^{q}\right)_{0 \leq a \leq m, q \in I_{M}},\left(x_{t_{a}^{(m)}}^{q}\right)_{0 \leq a \leq m, q \in I_{M}}\right)$. We find that for any $m \geq 1$,

$$
\lim _{\mathfrak{n} \rightarrow \infty} \varlimsup_{N \rightarrow \infty} \sup _{1 \leq i \leq C_{\mathfrak{n}}^{N}} \sup _{\mu, \nu \in \mathcal{V}_{i}^{N}} \sup _{f \in \mathfrak{U}}\left|\mathbb{E}^{\mu}[\hat{f}]-\mathbb{E}^{\nu}[\hat{f}]\right|=0 .
$$

Proof. We notice that for any $1 \leq i \leq C_{\mathfrak{n}}^{N}$ and any $\mu \in \mathcal{V}_{i}^{N}$,

$$
\mathbb{E}^{\mu(\boldsymbol{\alpha}, \mathbf{x})}\left[\hat{f}(\boldsymbol{\alpha}, \mathbf{x}) \chi\left\{\sup _{q \in I_{M}, 0 \leq a \leq m}\left|x_{a}^{q}\right| \geq \mathfrak{n}\right\}\right] \leq \mathfrak{n}^{-1} \mathbb{E}^{\mu(\boldsymbol{\alpha}, \mathbf{x})}\left[\sum_{a=0}^{m}\left\|\mathbf{x}_{a}\right\|^{2} \chi\left\{\sup _{q \in I_{M}, 0 \leq a \leq m}\left|x_{a}^{q}\right| \geq \mathfrak{n}\right\}\right] \leq 3(m+1) \mathfrak{n}^{-1},
$$

using the fact that $\mathcal{V}_{i}^{N} \subset \hat{\mathcal{W}}_{2}$ (as defined in (102)). Thus the mass assigned to non-bounded sets goes to zero uniformly as $\mathfrak{n} \rightarrow 0$. Furthermore it can be seen from the definition in (99) that the radius of the bounded sets goes to zero uniformly as $\mathfrak{n} \rightarrow \infty$, which implies the lemma.

Next we observe that the number of sets in the partition is subexponential in $N$ : this is an essential property, because it means that the partition size is dominated by the exponential decay of the probabilities in coming sections.

Lemma 4.2. For any $\mathfrak{n} \in \mathbb{Z}^{+}$,

$$
\varlimsup_{N \rightarrow \infty} N^{-1} \log C_{\mathfrak{n}}^{N}=0
$$

Proof. We notice from (104) that each $\mathcal{V}_{i}^{N}$ can assign $(N+1)$ possible values to the mass of each set $\hat{D}_{q} \times \hat{D}_{r} \in \mathcal{E}^{M} \times \mathbb{R}^{M}$. Since there are $\tilde{C}_{\mathfrak{n}}^{2}$ such sets, the number of such $\mathcal{V}_{i}^{N}$ must be upperbounded by $(N+1)^{\tilde{C}_{\mathrm{n}}^{2}} \mid$. Since this is polynomial in $N$, we have established the lemma.

\subsubsection{Definition of the Approximating Process}

We are now in a position to define the adapted stochastic process $\tilde{\boldsymbol{\sigma}}_{i}$ (for each $1 \leq i \leq C_{\mathfrak{n}}^{N}$ ), written $\tilde{\boldsymbol{\sigma}}_{i}:=$ $\left(\tilde{\sigma}_{i, t}^{q, j}\right)_{q \in I_{M}, j \in I_{N}, t \in[0, T]}$. Write $\tilde{\mathcal{V}}_{i, t}^{N} \subset \mathcal{M}_{1}^{+}\left(\mathcal{D}\left([0, t], \mathcal{E}^{M}\right)\right)$ to be the projection of the probability measures in $\mathcal{M}_{1}^{+}\left(\mathcal{D}\left([0, T], \mathcal{E}^{M} \times \mathbb{R}^{M}\right)\right)$ onto their marginals over $\mathcal{D}\left([0, t], \mathcal{E}^{M}\right)$ - and define $\mathcal{V}_{i, t}^{N}$ to be the analogous projection onto the marginal over $\mathcal{D}\left([0, t], \mathcal{E}^{M} \times \mathbb{R}^{M}\right)$. We write the intensity of $\tilde{\sigma}_{i, t}^{q, j}$ as $\tilde{\mathfrak{G}}_{i, t}^{q, j}$. We will choose the intensities to be such that as long as $\tilde{\mu}_{[0, t]}^{N}(\tilde{\boldsymbol{\sigma}}):=N^{-1} \sum_{j \in I_{N}} \delta_{\boldsymbol{\sigma}_{[0, t]}^{j}} \in \tilde{\mathcal{V}}_{i, t}^{N}$, then necessarily $\tilde{\mu}_{[0, t]}^{N}\left(\tilde{\boldsymbol{\sigma}}_{i}, \tilde{\mathfrak{G}}_{i}\right) \epsilon$ $\mathcal{V}_{i, t}^{N}$. This property is essential for us to be able to control the Girsanov Exponent in the next section.

We first find any set of paths $\boldsymbol{\alpha}_{i}$ and intensities $\mathfrak{G}_{i}$ that are such that their empirical process is in $\mathcal{V}_{i}^{N}$.

Lemma 4.3. For each $1 \leq i \leq C_{\mathfrak{n}}^{N}$, there exists $\boldsymbol{\alpha}_{i} \in \mathcal{D}\left([0, T], \mathcal{E}^{M}\right)^{N}$ and $\mathfrak{G}_{i} \in \mathcal{D}\left([0, T], \mathbb{R}^{M}\right)^{N}$ such that

$$
\begin{aligned}
\tilde{\mu}^{N}\left(\boldsymbol{\alpha}_{i}, \mathfrak{G}_{i}\right) & :=N^{-1} \sum_{j \in I_{N}} \delta_{\left(\boldsymbol{\alpha}_{i}^{j}, \mathfrak{G}_{i}^{j}\right)} \in \mathcal{V}_{i}^{N} \text { where } \boldsymbol{\alpha}_{i}=\left(\boldsymbol{\alpha}_{i}^{j}\right)_{j \in I_{N}}, \mathfrak{G}_{i}=\left(\mathfrak{G}_{i}^{j}\right)_{j \in I_{N}} \text { and } \\
\mathfrak{G}_{i, t} & =\mathfrak{G}_{i, t_{a}^{(m)}} \text { for all } t \in\left[t_{a}^{(m)}, t_{a+1}^{(m)}\right) \\
\boldsymbol{\alpha}_{i, t} & =\boldsymbol{\alpha}_{i, t_{a}^{(m)}} \text { for all } t \in\left[t_{a}^{(m)}, t_{a+1}^{(m)}\right)
\end{aligned}
$$

Proof. Let $\breve{\pi}: \mathcal{M}_{1}^{+}\left(\mathcal{D}\left([0, T], \mathcal{E}^{M} \times \mathbb{R}^{M}\right)\right) \rightarrow \mathcal{M}_{1}^{+}\left(\mathcal{E}^{M N(m+1)} \times \mathbb{R}^{M N(m+1)}\right)$ be the projection of a measure onto its marginal at times $\left\{t_{a}^{(m)}\right\}_{0 \leq a \leq m}$. Because empirical measures are dense in $\mathcal{M}_{1}^{+}\left(\mathcal{E}^{M N(m+1)} \times \mathbb{R}^{M N(m+1)}\right)$, 
for all large enough $N$, there must exist $\tilde{\boldsymbol{\alpha}}_{i} \in \mathcal{E}^{M N(m+1)}$, written $\tilde{\boldsymbol{\alpha}}_{i}:=\left(\tilde{\boldsymbol{\alpha}}_{i, a}\right)_{0 \leq a \leq m}$, and $\tilde{\mathfrak{G}}_{i} \in \mathbb{R}^{M N(m+1)}$, written $\tilde{\mathfrak{G}}_{i}:=\left(\tilde{\mathfrak{G}}_{i, a}\right)_{0 \leq a \leq m}$ such that

$$
\hat{\mu}^{N}\left(\tilde{\boldsymbol{\alpha}}_{i}, \tilde{\mathfrak{G}}_{i}\right):=N^{-1} \sum_{j \in I_{N}} \delta_{\left(\tilde{\boldsymbol{\alpha}}_{i}^{j}, \tilde{\mathfrak{G}}_{i}^{j}\right)} \in \breve{\pi} \cdot \mathcal{V}_{i}^{N}
$$

We can now define $\boldsymbol{\alpha}_{i}:=\left(\boldsymbol{\alpha}_{i, t}\right)_{t \in[0, T]} \in \mathcal{D}\left([0, T], \mathcal{E}^{M}\right)^{N}$ and $\mathfrak{G}_{i}:=\left(\mathfrak{G}_{i, t}\right)_{t \in[0, T]} \in \mathcal{D}\left([0, T], \mathcal{E}^{M}\right)^{N}$ as follows: for each $0 \leq a \leq m$,

$$
\begin{array}{lll}
\boldsymbol{\alpha}_{i, t_{a}^{(m)}}:=\tilde{\boldsymbol{\alpha}}_{i, a}, & \text { and } & \boldsymbol{\alpha}_{i, t}=\boldsymbol{\alpha}_{i, t_{a}^{(m)}} \text { for } t \in\left[t_{a}^{(m)}, t_{a+1}^{(m)}\right) \\
\mathfrak{G}_{i, t_{a}^{(m)}}:=\tilde{\mathfrak{G}}_{i, a}, & \text { and } & \mathfrak{G}_{i, t}=\mathfrak{G}_{i, t_{a}^{(m)}} \text { for all } t \in\left[t_{a}^{(m)}, t_{a+1}^{(m)}\right) .
\end{array}
$$

Next, we prove that if $\tilde{\mu}_{[0, t]}^{N}(\tilde{\boldsymbol{\sigma}}) \in \tilde{\mathcal{V}}_{i, t}^{N}$, then we must be able to find a permutation of the intensities $\left\{\mathfrak{G}_{i, t}^{j}\right\}$ that ensures that the associated empirical process is in $\mathcal{V}_{i}^{N}$. Define $\mathfrak{P}^{N}$ to be the set of all permutations on $I_{N}$ (i.e. each member of $\mathfrak{P}^{N}$ is a bijective map $I_{N} \rightarrow I_{N}$ ).

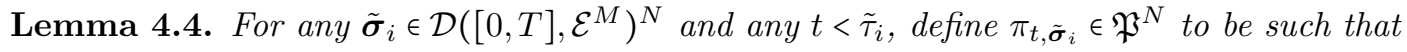

$$
\begin{aligned}
\tilde{\sigma}_{i, t_{a}^{(m)}}^{q, j} & =\alpha_{i, t_{a}^{(m)}}^{q, \pi_{t, \tilde{\sigma}_{i}}(j)} \text { for all } t_{a}^{(m)}<\tilde{\tau}_{i} \text { and } \\
\pi_{t} & =\pi_{t_{a}^{(m)}} \text { for all } t \in\left[t_{a}^{(m)}, t_{a+1}^{(m)}\right) .
\end{aligned}
$$

$\pi_{t, \tilde{\boldsymbol{\sigma}}}$ is well-defined, but not uniquely defined. Furthermore $\pi_{,,,}: \mathcal{D}\left([0, T], \mathcal{E}^{M}\right)^{N} \times[0, T] \rightarrow \mathfrak{P}^{N}$ is progressivelymeasurable

Proof. Write $\breve{\boldsymbol{\alpha}}_{i, t}:=\boldsymbol{\alpha}_{i, t \wedge \tilde{\tau}_{i}}$. We first claim that $\breve{\pi} \cdot \tilde{\mu}^{N}\left(\tilde{\boldsymbol{\sigma}}_{i}\right)=\breve{\pi} \cdot \tilde{\mu}^{N}\left(\breve{\boldsymbol{\alpha}}_{i}\right)$, as long as $t<\tilde{\tau}_{i}$. This is because $\mathcal{V}_{i}^{N}$ specifies the mass of each set to an accuracy of $N^{-1}$, but the mass assigned to any set by the empirical measure must also be a multiple of $N^{-1}$. This means that we must be able to find a permutation such that (112) is satisfied.

We can now formally define the stochastic process $\tilde{\boldsymbol{\sigma}}_{i}$. First, $\tilde{\sigma}_{i, t}^{q, j}$ is 'stopped' once the empirical measure is no longer in $\tilde{\mathcal{V}}_{i, t}^{N}$, i.e.

$$
\begin{aligned}
\tilde{\sigma}_{i, t}^{q, j} & :=\tilde{\sigma}_{i, \tilde{\tau}_{i}}^{q, j} \text { where } \\
\tilde{\tau}_{i} & =\inf \left\{t \geq 0: \tilde{\mu}_{[0, t]}^{N}(\tilde{\boldsymbol{\sigma}}) \notin \tilde{\mathcal{V}}_{i, t}^{N}\right\} \text { and } \tilde{\mu}_{[0, t]}^{N}(\tilde{\boldsymbol{\sigma}}):=N^{-1} \sum_{j \in I_{N}} \delta_{\boldsymbol{\sigma}_{[0, t]}^{j}} \in \mathcal{M}_{1}^{+}\left(\mathcal{D}\left([0, t], \mathcal{E}^{M}\right)\right) .
\end{aligned}
$$

For all $t \leq \tilde{\tau}_{i}$, we stipulate that $\tilde{\sigma}_{i, t}^{q, j}$ satisfies the identity,

$$
\tilde{\sigma}_{i, t}^{q, j}=\sigma_{0}^{q, j} A \cdot Y^{q, j}\left(\int_{0}^{t} c\left(\tilde{\sigma}_{i, s}^{q, j}, \mathfrak{G}_{i, s}^{q, \pi_{s, \tilde{\sigma}_{i}}(j)}\right) d s\right),
$$

recalling that $A \cdot x$ is defined to be $-1^{x}$. Recall from (114) that $\tilde{\sigma}_{t}^{q, j}$ is defined to be stopped for $t \geq \tilde{\tau}_{i}$.

Lemma 4.5. The stochastic processes $\left\{\tilde{\sigma}_{i, t}^{q, j}\right\}_{j \in I_{N}, q \in I_{M}, t \in[0, T]}$ are uniquely well-defined and are adapted to the

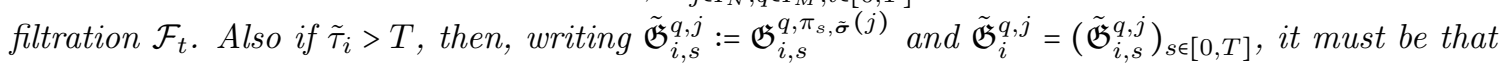

$$
\tilde{\mu}^{N}\left(\tilde{\boldsymbol{\sigma}}_{i}, \tilde{\mathfrak{G}}_{i}\right) \in \mathcal{V}_{i}^{N}
$$

Proof. This is immediate from the definitions. 


\subsection{Girsanov's Theorem}

In this section we demonstrate that the probability law of the original system $\sigma_{t}$ can be well-approximated by the law of one of the processes $\left\{\tilde{\boldsymbol{\sigma}}_{i, t}\right\}_{1 \leq i \leq C_{n}^{N}}$. The main result is Lemma 4.6: the implication of this lemma is that if we can show that the flow operator accurately describes the dynamics of the empirical processes generated by each of the $\tilde{\boldsymbol{\sigma}}_{i}$, then it must accurately describe the original empirical process as well.

Let $R_{i}^{N} \in \mathcal{M}_{1}^{+}\left(\mathcal{D}\left([0, T], \mathcal{E}^{M}\right)^{N}\right)$ be the probability law of the processes $\left\{\tilde{\sigma}_{i, t}^{q, j}\right\}_{j \in I_{N}, q \in I_{M}, t \in[0, T]}$. Define the stopping time $\tau_{i}$ that is the analog of $\tilde{\tau}_{i}$ in (115), i.e.

$$
\tau_{i}=\inf \left\{t \geq 0: \tilde{\mu}_{[0, t]}^{N}(\sigma) \notin \pi_{t} \cdot \tilde{\mathcal{V}}_{i, t}^{N}\right\} .
$$

Notice that, necessarily,

$$
\tau_{i} \in\left\{t_{a}^{(m)}\right\}_{0 \leq a \leq m}
$$

Let $P_{\mathbf{J}}^{N} \in \mathcal{M}_{1}^{+}\left(\mathcal{D}\left([0, T], \mathcal{E}^{M}\right)^{N}\right)$ be the law of the original spin system $\left\{\sigma_{i, t \wedge \tau_{i} \wedge T}^{q, j}\right\}_{j \in I_{N}, q \in I_{M}, t \in[0, T]}$, conditioned on a realization of the connections $\mathbf{J}$, and stopped at time $\tau_{i}$. Write

$$
\hat{\mathfrak{G}}_{i, s}^{q, j}:=\mathfrak{G}_{i, s}^{q, \pi_{s, \sigma}(j)},
$$

where $\pi .$, is defined in Lemma 4.4. Define the Girsanov exponent

$$
\begin{aligned}
\Gamma_{i}^{N}\left(\boldsymbol{\sigma}_{[0, T]}, \mathbf{J}\right)=N^{-1} \sum_{q \in I_{M}, j \in I_{N}}\left\{\int_{0}^{\tau_{i} \wedge T}\left\{c\left(\sigma_{s}^{q, j}, \hat{\mathfrak{G}}_{i, s}^{q, j}\right)-c\left(\sigma_{s}^{q, j}, G_{s}^{q, j}\right)\right\} d s\right. & \\
& \left.+\int_{0}^{\tau_{i} \wedge T}\left\{\log c\left(\sigma_{s}^{q, j}, G_{s}^{q, j}\right)-\log c\left(\sigma_{s}^{q, j}, \hat{\mathfrak{G}}_{i, s}^{q, j}\right)\right\} d \hat{\sigma}_{s}^{q, j}\right\},
\end{aligned}
$$

and we have defined $\hat{\sigma}_{s}^{i, j}$ to be the integer-valued nondecreasing càdlàg process specifying how many times that $\sigma_{s}^{i, j}$ has changed sign over the time period [0,s), i.e. $\sigma_{s}^{i, j}=\sigma_{0}^{i, j} \times(-1)^{\hat{\sigma}_{s}^{i, j}}$. It follows from Girsanov's Theorem ${ }^{4}[37,43]$ that the Radon-Nikodym derivative satisfies

$$
\frac{d P_{\mathbf{J}}^{N}}{d R_{i}^{N}}\left(\boldsymbol{\sigma}_{[0, T]}\right)=\exp \left(N \Gamma_{i}^{N}\left(\boldsymbol{\sigma}_{[0, T]}, \mathbf{J}\right)\right) .
$$

Write $\tilde{G}_{i, t}^{q, j}=N^{-1 / 2} \sum_{k \in I_{N}} J^{j k} \tilde{\sigma}_{i, t}^{q, k}$ and define $\tilde{\tau}_{N}$ to be the analog of (21),i.e.

$$
\tilde{\tau}_{N}=T \wedge \inf \left\{t: t \in[0, T] \text { and } \boldsymbol{\sigma}_{t} \notin \mathcal{X}^{N}\right\} .
$$

Lemma 4.6. Suppose that for any $\bar{\epsilon}>0$, there exists $n_{0} \in \mathbb{Z}^{+}$such that for all $n \geq n_{0}$, there exists $\mathfrak{n}_{0}(n) \in \mathbb{Z}^{+}$, such that for all $\mathfrak{n} \geq \mathfrak{n}_{0}(n)$, there exists $m_{0}(n, \mathfrak{n})$ such that for all $m \geq m(n, \mathfrak{n})$,

$$
\begin{aligned}
& \sup _{0 \leq b<n} \sup _{1 \leq i \leq C_{\mathfrak{n}}^{N}} \varlimsup_{N \rightarrow \infty} N^{-1} \log \mathbb{P}\left(\mathcal{J}_{N}, \tilde{\tau}_{N}>t_{b}^{(n)}, \tilde{\mu}^{N}\left(\tilde{\boldsymbol{\sigma}}_{i}, \tilde{\mathbf{G}}_{i}\right) \in \mathcal{V}_{i}^{N}\right. \text { and } \\
&\left.d_{W}\left(\xi_{b}\left(\tilde{\boldsymbol{\sigma}}_{i, t_{b}^{(n)}}, \tilde{\mathbf{G}}_{i, t_{b}^{(n)}}\right), \hat{\mu}^{N}\left(\tilde{\boldsymbol{\sigma}}_{i, t_{b+1}^{(n)}}, \tilde{\mathbf{G}}_{i, t_{b+1}^{(n)}}\right)\right) \geq \bar{\epsilon} T n^{-1}\right):=-\mathfrak{k}<0,
\end{aligned}
$$

for some $\mathfrak{k}>0$. Then the condition of Lemma 3.5 is satisfied, i.e. for any $\tilde{\epsilon}>0$, for large enough $n \in \mathbb{Z}^{+}$,

$$
\varlimsup_{N \rightarrow \infty} N^{-1} \log \mathbb{P}\left(\mathcal{J}_{N} \text { and } \tau_{N}>t_{b}^{(n)} \text { and } d_{W}\left(\xi_{b}\left(\boldsymbol{\sigma}_{t_{b}^{(n)}}, \mathbf{G}_{t_{b}^{(n)}}\right), \hat{\mu}^{N}\left(\boldsymbol{\sigma}_{t_{b+1}^{(n)}}, \mathbf{G}_{t_{b+1}^{(n)}}\right)\right) \geq \tilde{\epsilon} T n^{-1}\right)<0 .
$$

\footnotetext{
${ }^{4}$ A quick way to see why this formula holds is to note that the probability of a jump occurring over a small time interval is approximately exponentially distributed, i.e. $\mathbb{P}\left(\left|\sigma_{\Delta}^{i, j}-\sigma_{0}^{i, j}\right|>0\right) \simeq c\left(\sigma_{0}^{i, j}, G_{0}^{i, j}\right) \exp \left(-\Delta c\left(\sigma_{0}^{i, j}, G_{0}^{i, j}\right)\right)$. Taking the ratio of two such densities, multiplying over many time intervals, and then taking $\Delta \rightarrow 0$, we obtain the formula (122).
} 
Proof. The event $\mathcal{J}_{N}$ necessarily implies that $\tilde{\mu}^{N} \in \hat{\mathcal{W}}_{2}$. We can thus apply a union-of-events bound to the partition in (103) to obtain that

$$
\begin{aligned}
& \mathbb{P}\left(\mathcal{J}_{N}, \tau_{N}>t_{b}^{(n)}, d_{W}\left(\xi_{b}\left(\boldsymbol{\sigma}_{t_{b}^{(n)}}, \mathbf{G}_{t_{b}^{(n)}}\right), \hat{\mu}^{N}\left(\boldsymbol{\sigma}_{t_{b+1}^{(n)}}, \mathbf{G}_{t_{b+1}^{(n)}}\right)\right) \geq \tilde{\epsilon} \Delta\right) \\
& \begin{aligned}
\leq \sum_{i=1}^{C_{\mathfrak{n}}^{N}} \mathbb{P}\left(\mathcal{J}_{N}, \tau_{N}>t_{b}^{(n)}, d_{W}\left(\xi_{b}\left(\boldsymbol{\sigma}_{t_{b}^{(n)}}, \mathbf{G}_{t_{b}^{(n)}}\right), \hat{\mu}^{N}\left(\boldsymbol{\sigma}_{t_{b+1}^{(n)}}, \mathbf{G}_{t_{b+1}^{(n)}}\right)\right) \geq \tilde{\epsilon} \Delta, \tilde{\mu}^{N}(\boldsymbol{\sigma}, \mathbf{G}) \in \mathcal{V}_{i}^{N},\left|\Gamma_{i}^{N}(\boldsymbol{\sigma}, \mathbf{J})\right| \leq \mathfrak{k} / 2\right) \\
\quad+\sum_{i=1}^{C_{\mathfrak{n}}^{N}} \mathbb{P}\left(\mathcal{J}_{N}, \tilde{\mu}^{N}(\boldsymbol{\sigma}, \mathbf{G}) \in \mathcal{V}_{i}^{N},\left|\Gamma_{i}^{N}(\boldsymbol{\sigma}, \mathbf{J})\right|>\mathfrak{k} / 2\right), \quad(1
\end{aligned}
\end{aligned}
$$

noting that the constant $\mathfrak{k}$ is defined in (124). Noting that $C_{\mathfrak{n}}^{N}$ is polynomial in $N$ (as proved in Lemma 4.2), thanks to Lemma 3.1, it suffices to prove that each of the terms on the right hand side of (126) are exponentially decaying in $N$. Using the Radon-Nikodym derivative (122),

$$
\begin{aligned}
\mathbb{P}\left(\mathcal{J}_{N}, \tau_{N}>t_{b}^{(n)},\right. & \left.d_{W}\left(\xi_{b}\left(\boldsymbol{\sigma}_{t_{b}^{(n)}}, \mathbf{G}_{t_{b}^{(n)}}\right), \hat{\mu}^{N}\left(\boldsymbol{\sigma}_{t_{b+1}^{(n)}}, \mathbf{G}_{t_{b+1}^{(n)}}\right)\right) \geq \tilde{\epsilon} \Delta, \tilde{\mu}^{N}(\boldsymbol{\sigma}, \mathbf{G}) \in \mathcal{V}_{i}^{N},\left|\Gamma_{i}^{N}(\boldsymbol{\sigma}, \mathbf{J})\right| \leq \mathfrak{k} / 2\right) \\
& \leq \exp (N \mathfrak{k} / 2) \mathbb{P}\left(\mathcal{J}_{N}, \tilde{\tau}_{N}>t_{b}^{(n)}, d_{W}\left(\xi_{b}\left(\tilde{\boldsymbol{\sigma}}_{i, t_{b}^{(n)}}, \tilde{\mathbf{G}}_{i, t_{b}^{(n)}}\right), \hat{\mu}^{N}\left(\tilde{\boldsymbol{\sigma}}_{i, t_{b+1}^{(n)}}, \tilde{\mathbf{G}}_{i, t_{b+1}^{(n)}}\right)\right) \geq \tilde{\epsilon} \Delta, \tilde{\mu}^{N}\left(\tilde{\boldsymbol{\sigma}}_{i}, \tilde{\mathbf{G}}_{i}\right) \in \mathcal{V}_{i}^{N}\right) \\
& \leq \exp (-N \mathfrak{k} / 2),
\end{aligned}
$$

using the assumption (124) in the statement of the lemma. It thus remains to prove that

$$
\varlimsup_{N \rightarrow \infty} \sup _{1 \leq i \leq C_{\mathbf{n}}^{N}} N^{-1} \log \mathbb{P}\left(\mathcal{J}_{N}, \tilde{\mu}^{N}(\boldsymbol{\sigma}, \mathbf{G}) \in \mathcal{V}_{i}^{N},\left|\Gamma_{i}^{N}(\boldsymbol{\sigma}, \mathbf{J})\right|>\mathfrak{k} / 2\right)<0 .
$$

Notice that $\tilde{\mu}^{N}(\boldsymbol{\sigma}, \mathbf{G}) \in \mathcal{V}_{i}^{N}$ implies that $\tau_{i}>T$. Recalling that $\hat{\mathfrak{G}}_{a}^{q, j}:=\hat{\mathfrak{G}}_{t_{a}^{(m)}}^{q, j}$ and $\sigma_{a}^{q, j}:=\sigma_{t_{a}^{(n)}}^{q, j}$, define the following time-discretized approximation of the Girsanov Exponent,

$$
\begin{aligned}
\tilde{\Gamma}_{i}^{N}\left(\boldsymbol{\sigma}_{[0, T]}, \mathbf{J}\right) & =N^{-1} \sum_{q \in I_{M}, j \in I_{N}}\left\{\operatorname{Tm}^{-1} \sum_{a=0}^{m-1}\left\{c\left(\sigma_{a}^{q, j}, \hat{\mathfrak{G}}_{i, a}^{q, j}\right)-c\left(\sigma_{a}^{q, j}, G_{a}^{q, j}\right)\right\}\right. \\
& \left.-\frac{1}{2} \sum_{a=0}^{m-1}\left\{\chi\left\{\left|G_{a}^{q, j}\right| \leq \mathfrak{n}\right\} \log c\left(\sigma_{a}^{q, j}, G_{a}^{q, j}\right)-\chi\left\{\left|\hat{\mathfrak{G}}_{i, a}^{q, j}\right| \leq \mathfrak{n}\right\} \log c\left(\sigma_{a}^{q, j}, \hat{\mathfrak{G}}_{i, a}^{q, j}\right)\right\} \sigma_{a}^{q, j}\left(\sigma_{a+1}^{q, j}-\sigma_{a}^{q, j}\right)\right\} .
\end{aligned}
$$

One expects the above approximation to be very accurate for large $m \in \mathbb{Z}^{+}$because

$$
\hat{\sigma}_{a+1}^{q, j}-\hat{\sigma}_{a}^{q, j} \in\{0,1\} \text { implies that }-\frac{1}{2} \sigma_{a}^{q, j}\left(\sigma_{a+1}^{q, j}-\sigma_{a}^{q, j}\right)=\hat{\sigma}_{a+1}^{q, j}-\hat{\sigma}_{a}^{q, j} .
$$

(The probability that $\hat{\sigma}_{a+1}^{q, j}-\hat{\sigma}_{a}^{q, j} \geq 2$ is very small once the time interval $\mathrm{Tm}^{-1}$ is small). Thus to establish (127), it suffices to establish the follow two identities

$$
\begin{array}{r}
\varlimsup_{N \rightarrow \infty} \sup _{1 \leq i \leq C_{\mathfrak{n}}^{N}} N^{-1} \log \mathbb{P}\left(\mathcal{J}_{N}, \tilde{\mu}^{N}(\boldsymbol{\sigma}, \mathbf{G}) \in \mathcal{V}_{i}^{N}, \Gamma_{i}^{N}(\boldsymbol{\sigma}, \mathbf{J})-\tilde{\Gamma}_{i}^{N}(\boldsymbol{\sigma}, \mathbf{J})>\mathfrak{k} / 4\right)<0 \\
\varlimsup_{N \rightarrow \infty} \sup _{1 \leq i \leq C_{\mathfrak{n}}^{N}} N^{-1} \log \mathbb{P}\left(\mathcal{J}_{N}, \tilde{\mu}^{N}(\boldsymbol{\sigma}, \mathbf{G}) \in \mathcal{V}_{i}^{N},\left|\tilde{\Gamma}_{i}^{N}(\boldsymbol{\sigma}, \mathbf{J})\right|>\mathfrak{k} / 4\right)<0 .
\end{array}
$$

We start by establishing (131). We observe from (128) that there exists a function $\mathcal{H}: \mathcal{D}\left([0, T], \mathcal{E}^{M} \times \mathbb{R}^{M}\right) \rightarrow \mathbb{R}$ such that

$$
\tilde{\Gamma}_{i}^{N}\left(\boldsymbol{\sigma}_{[0, T]}, \mathbf{J}\right)=\mathbb{E}^{\tilde{\mu}^{N}(\boldsymbol{\sigma}, \mathbf{G})}[\mathcal{H}]-\mathbb{E}^{\tilde{\mu}^{N}\left(\boldsymbol{\sigma}, \hat{\mathfrak{G}}_{i}\right)}[\mathcal{H}] .
$$

Furthermore $\mathcal{H}$ is a function of the values of the variables at the times $\left\{t_{a}^{(m)}\right\}_{0 \leq a \leq m}$. Now if $\tilde{\mu}^{N}(\boldsymbol{\sigma}, \mathbf{G}) \in \mathcal{V}_{i}^{N}$, then necessarily $\tilde{\mu}^{N}(\boldsymbol{\sigma}, \hat{\mathfrak{G}}) \in \mathcal{V}_{i}^{N}$. It now follows from (i) the fact that the functions $c$ and $\log c$ are uniformly Lipschitz in their second argument and (ii) Lemma 4.1, that for large enough $\mathfrak{n}$, it must be that

$$
\left|\mathbb{E}^{\tilde{\mu}^{N}(\boldsymbol{\sigma}, \mathbf{G})}[\mathcal{H}]-\mathbb{E}^{\tilde{\mu}^{N}\left(\boldsymbol{\sigma}, \hat{\mathfrak{E}}_{i}\right)}[\mathcal{H}]\right| \leq \mathfrak{k} / 4
$$


We have thus established (131). It remains to establish (130). Write

$$
\begin{aligned}
F_{s}^{q, j} & =\chi\left\{-\mathfrak{n} \leq G_{s}^{q, j} \leq \mathfrak{n}\right\} \log c\left(\sigma_{s}^{q, j}, G_{s}^{q, j}\right)-\chi\left\{-\mathfrak{n} \leq \mathfrak{G}_{s}^{q, j} \leq \mathfrak{n}\right\} \log c\left(\sigma_{s}^{q, j}, \hat{\mathfrak{G}}_{i, s}^{q, j}\right) \\
f_{s}^{q, j} & =\chi\left\{\left|G_{s}^{q, j}\right|>\mathfrak{n}\right\} \log c\left(\sigma_{s}^{q, j}, G_{s}^{q, j}\right) .
\end{aligned}
$$

We wish to split $\Gamma_{i}^{N}(\boldsymbol{\sigma}, \mathbf{J})-\tilde{\Gamma}_{i}^{N}(\boldsymbol{\sigma})$ into the sum of five terms and bound each term separately. First, using (129), we notice that the difference of the stochastic integral in $\Gamma_{i}^{N}(\sigma, \mathbf{J})$ and its time-discretized equivalent in $\tilde{\Gamma}_{i}^{N}(\boldsymbol{\sigma})$ is

$$
\int_{t_{a}^{(n)}}^{t_{a+1}^{(m)}} F_{t_{a}^{(m)}}^{q, j} d \hat{\sigma}_{s}^{q, j}+\frac{1}{2} F_{t_{a}^{(m)}}^{q, j} \sigma_{a}^{q, j}\left(\sigma_{a+1}^{q, j}-\sigma_{a}^{q, j}\right)=F_{t_{a}^{(m)}}^{q, j}\left(\hat{\sigma}_{t_{a+1}^{(m)}}^{q, j}-\hat{\sigma}_{t_{a}^{(m)}}^{q, j}\right) \chi\left\{\hat{\sigma}_{t_{a+1}^{(m)}}^{q, j}-\hat{\sigma}_{t_{a}^{(m)}}^{q, j} \geq 2\right\} .
$$

Second, it is immediate from the definition that it is always the case that $-\mathfrak{n} \leq \hat{\mathfrak{G}}_{s}^{q, j} \leq \mathfrak{n}$. In order that (130) is satisfied, it suffices to demonstrate the following identities,

$$
\begin{aligned}
& \varlimsup_{N \rightarrow \infty} N^{-1} \log \mathbb{P}\left(\mathcal{J}_{N}, \sum_{j \in I_{N}, q \in I_{M}} \int_{0}^{T} f_{s}^{q, j} d \hat{\sigma}_{s}^{q, j} \geq \frac{N \mathfrak{k}}{20}\right)<0 \\
& \varlimsup_{N \rightarrow \infty} N^{-1} \log \mathbb{P}\left(\mathcal{J}_{N}, \sum_{j \in I_{N}, q \in I_{M}} \sum_{a=0}^{m-1} \int_{t_{a}^{(m)}}^{t_{a+1}^{(m)}}\left(F_{s}^{q, j}-F_{t_{a}^{(m)}}^{q, j}\right)\left(d \hat{\sigma}_{s}^{q, j}-c\left(\sigma_{s}^{q, j}, G_{s}^{q, j}\right) d s\right) \geq \frac{N \mathfrak{k}}{20}\right)<0 \\
& \varlimsup_{N \rightarrow \infty} N^{-1} \log \mathbb{P}\left(\mathcal{J}_{N}, \tilde{\mu}^{N}(\boldsymbol{\sigma}, \mathbf{G}) \in \mathcal{V}_{i}^{N}, \sum_{j \in I_{N}, q \in I_{M}} \sum_{a=0}^{m-1} \int_{t_{a}^{(m)}}^{t_{a+1}^{(m)}}\left(F_{s}^{q, j}-F_{t_{a}^{q, j}}^{q, j}\right) c\left(\sigma_{s}^{q, j}, G_{s}^{q, j}\right) d s \geq \frac{N \mathfrak{k}}{20}\right)<0 \\
& \varlimsup_{N \rightarrow \infty} N^{-1} \log \mathbb{P}\left(\mathcal{J}_{N}, \sum_{a=0}^{m-1} \sum_{q \in I_{M}, j \in I_{N}} F_{\left.t_{a}^{q, j}\right)}^{q, j}\left(\hat{\sigma}_{t_{a+1}^{q, j}}^{q, j}-\hat{\sigma}_{t_{a}^{(m)}}^{q, j}\right) \chi\left\{\hat{\sigma}_{t_{a+1}^{q, j}}^{(m)}-\hat{\sigma}_{t_{a}^{(m)}}^{q, j} \geq 2\right\} \geq \frac{N \mathfrak{k}}{20}\right)<0 \\
& \varlimsup_{N \rightarrow \infty} N^{-1} \log \mathbb{P}\left(\mathcal{J}_{N}, \tilde{\mu}^{N}(\boldsymbol{\sigma}, \mathbf{G}) \in \mathcal{V}_{i}^{N} \text { and } \mid \sum_{q \in I_{M}, j \in I_{N}} \sum_{a=0}^{m-1}\left\{\frac{T c\left(\sigma_{a}^{q, j}, \hat{\mathfrak{G}}_{i, a}^{q, j}\right)-T c\left(\sigma_{a}^{q, j}, G_{a}^{q, j}\right)}{m}\right.\right. \\
& \left.\left.-\int_{t_{a}^{(m)}}^{t_{a+1}^{(m)}}\left\{c\left(\sigma_{s}^{q, j}, \hat{\mathfrak{G}}_{i, s}^{q, j}\right)-c\left(\sigma_{s}^{q, j}, G_{s}^{q, j}\right)\right\} d s\right\} \mid>\frac{N \mathfrak{k}}{20}\right)<0 .
\end{aligned}
$$

We start with (133). The event $\mathcal{J}_{N}$ implies that $N^{-1} \sum_{j \in I_{N}} \chi\left\{\left|G_{s}^{q, j}\right|>\mathfrak{n}\right\} \leq 3 \mathfrak{n}^{-2}$. Thus, since $c(\cdot, \cdot)$ is uniformly upperbounded by $c_{1}$,

$$
N^{-1} \sum_{q \in I_{M}, j \in I_{N}} \chi\left\{\left|G_{s}^{q, j}\right|>\mathfrak{n}\right\} \exp \left(f_{s}^{q, j}\right) \leq 3 M \mathfrak{n}^{-2} \log \left(c_{1}\right) .
$$

Since the right hand side goes to zero as $\mathfrak{n} \rightarrow \infty$, (133) follows from (ii) of Lemma 8.2, as long as $\mathfrak{n}$ is large enough.

(134) follows from the concentration inequality in (i) of Lemma 8.2, employing the facts that (i) $\left|F_{s}^{q, j}\right|$ is uniformly upperbounded, and (ii) $\hat{\sigma}_{t}^{q, j}-\int_{0}^{t} c\left(\sigma_{s}^{q, j}, G_{s}^{q, j}\right) d s$ is a compensated Poisson Process (a Martingale $[2])$.

For (135), the boundedness of $c(\cdot, \cdot)$ by $c_{1}$ (in the first line), and Jensen's Inequality (in the second line) imply that

$$
\begin{aligned}
N^{-1}\left|\sum_{j \in I_{N}, q \in I_{M}} \sum_{a=0}^{m-1} \int_{t_{a}^{(m)}}^{t_{a+1}^{(m)}}\left(F_{s}^{q, j}-F_{t_{a}^{(m)}}^{q, j}\right) c\left(\sigma_{s}^{q, j}, G_{s}^{q, j}\right) d s\right| & \leq N^{-1} c_{1} \sum_{j \in I_{N}, q \in I_{M}} \sum_{a=0}^{m-1} \int_{t_{a}^{(m)}}^{t_{a+1}^{(m)}}\left|F_{s}^{q, j}-F_{t_{a}^{(m)}}^{q, j}\right| d s \\
& \leq c_{1} \int_{0}^{T}\left\{N^{-1} \sum_{j \in I_{N}, q \in I_{M}}\left|F_{t}^{q, j}-F_{t^{(m)}}^{q, j}\right|^{2}\right\}^{1 / 2} d t \\
& \leq c_{1} \sqrt{c_{L}} \sqrt{3} \int_{0}^{T}\left\{N^{-1} \sum_{j \in I_{N}, q \in I_{M}}\left|\sigma_{t}^{q, j}-\sigma_{t^{(m)}}^{q, j}\right|^{2}\right\}^{1 / 2} d t,
\end{aligned}
$$


using (i) the fact that $\log c(\cdot, \cdot)$ has Lipschitz constant $c_{L}$ (in its second argument), and (ii) as long as the event $\mathcal{J}_{N}$ holds. Define the renewed Poisson Processes $\left\{Y_{a}^{q, j}(t)\right\}_{q \in I_{M}, j \in I_{N}}$ to be

$$
Y_{a}^{q, j}(t):=Y^{q, j}\left(t+\int_{0}^{t_{a}^{(m)}} c\left(\sigma_{s}^{q, j}, G_{s}^{q, j}\right) d s\right)-Y^{q, j}\left(\int_{0}^{t_{a}^{(m)}} c\left(\sigma_{s}^{q, j}, G_{s}^{q, j}\right) d s\right) .
$$

Now since the flipping intensity is uniformly upperbounded by $c_{1}$, if $t \leq t_{a+1}^{(m)}$ then

$$
\begin{aligned}
\sum_{q \in I_{M}, j \in I_{N}}\left|\sigma_{t}^{q, j}-\sigma_{t_{a}^{(m)}}^{q, j}\right|^{2} & \leq 4 \sum_{q \in I_{M}, j \in I_{N}} \chi\left\{\hat{Y}_{a}^{q, j}\left(c_{1} t-c_{1} t_{a}^{(m)}\right) \geq 1\right\} \text { where } \\
\hat{Y}_{a}^{q, j}(t) & =Y_{a}^{q, j}\left(t \wedge \hat{\tau}_{a}^{q, j}\right) \text { and } \\
\hat{\tau}_{a}^{q, j} & =\inf \left\{u \geq 0: u=\int_{t_{a}^{(m)}}^{t_{a+1}^{(m)}} c\left(\sigma_{s}^{q, j}, G_{s}^{q, j}\right) d s\right\} .
\end{aligned}
$$

Now $t-t^{(m)} \leq \delta$, where $\delta=T m^{-1}$. Jensen's Inequality thus implies that

$$
\int_{0}^{T}\left\{N^{-1} \sum_{j \in I_{N}, q \in I_{M}} \chi\left\{\hat{Y}_{a}^{q, j}\left(c_{1} \delta\right) \geq 1\right\}\right\}^{1 / 2} d t \leq \sqrt{T}\left\{\int_{0}^{T} N^{-1} \sum_{j \in I_{N}, q \in I_{M}} \chi\left\{\hat{Y}_{a}^{q, j}\left(c_{1} \delta\right) \geq 1\right\} d t\right\}^{1 / 2} .
$$

We thus find that there is a constant $C$ such that

$$
\begin{array}{r}
\mathbb{P}\left(\mathcal{J}_{N}, \tilde{\mu}^{N}(\boldsymbol{\sigma}, \mathbf{G}) \in \mathcal{V}_{i}^{N}, \sum_{j \in I_{N}, q \in I_{M}} \sum_{a=0}^{m-1} \int_{t_{a}^{(m)}}^{t_{a+1}^{(m)}}\left(F_{s}^{q, j}-F_{t_{a}^{(m)}}^{q, j}\right) c\left(\sigma_{s}^{q, j}, G_{s}^{q, j}\right) d s \geq \frac{N \mathfrak{k}}{16}\right) \\
\leq \mathbb{P}\left(N^{-1} \sum_{a=0}^{m-1} \sum_{j \in I_{N}, q \in I_{M}} \chi\left\{\hat{Y}_{a}^{q, j}\left(c_{1} \delta\right) \geq 1\right\} \geq C\right) .
\end{array}
$$

For large enough $m$, this probability is exponentially decaying, thanks to Lemma 8.1.

For (136), since the flipping rate is uniformly upperbounded by $c_{1}$, there exists a constant $C(\mathfrak{n})$ such that $F_{S}^{q, j} \leq C(\mathfrak{n})$. Thus by Chernoff's Inequality,

$$
\begin{aligned}
\mathbb{P}\left(\mathcal{J}_{N}, \sum_{a=0}^{m-1} \sum_{q \in I_{M}, j \in I_{N}} F_{t_{a}^{q(m)}}^{q, j}\left(\hat{\sigma}_{t_{a+1}^{q, j}}^{q, j}-\hat{\sigma}_{t_{a}^{(m)}}^{q, j}\right) \chi\left\{\hat{\sigma}_{t_{a+1}^{q, j}}^{q, j}-\hat{\sigma}_{t_{a}^{(m)}}^{q, j} \geq 2\right\} \geq \frac{N \mathfrak{k}}{20}\right) \\
\leq \mathbb{P}\left(\sum_{a=0}^{m-1} \sum_{q \in I_{M}, j \in I_{N}} \hat{Y}_{a}^{q, j}\left(c_{1} \delta\right) \chi\left\{\hat{Y}_{a}^{q, j}\left(c_{1} \delta\right) \geq 2\right\} \geq \frac{N \mathfrak{k}}{16 C(\mathfrak{n})}\right) \\
\leq \mathbb{E}\left[\exp \left(v \sum_{a=0}^{m-1} \sum_{q \in I_{M}, j \in I_{N}} \hat{Y}_{a}^{q, j}\left(c_{1} \delta\right) \chi\left\{\hat{Y}_{a}^{q, j}\left(c_{1} \delta\right) \geq 2\right\}-\frac{N \mathfrak{k} v}{20 C(\mathfrak{n})}\right)\right],
\end{aligned}
$$

for some constant $v>0$. To bound (139), we start by evaluating the integral conditionally on $\mathcal{F}_{t_{m-1}^{(m)}}$. Notice that $\left\{\hat{Y}_{m-1}^{q, j}\right\}_{q \in I_{M}, j \in I_{N}}$ are independent of $\mathcal{F}_{t_{m-1}^{(m)}}$ (thanks to the renewal property of Poisson Processes). Also $\hat{Y}_{a}^{q, j}\left(c_{1} \delta\right) \chi\left\{\hat{Y}_{a}^{q, j}\left(c_{1} \delta\right) \geq 2\right\} \leq Y_{a}^{q, j}\left(c_{1} \delta\right) \chi\left\{Y_{a}^{q, j}\left(c_{1} \delta\right) \geq 2\right\}$. We thus find that, for $a=m-1$, and using the fact that $\mathbb{P}\left(Y_{a}^{q, j}\left(c_{1} \delta\right)=r\right)=\exp \left(-r \delta c_{1}\right)\left(\delta c_{1}\right)^{r} /(r !)$,

$$
\mathbb{E}\left[\exp \left(v \sum_{q \in I_{M}, j \in I_{N}} \hat{Y}_{a}^{q, j}\left(c_{1} \delta\right) \chi\left\{\hat{Y}_{a}^{q, j}\left(c_{1} \delta\right) \geq 2\right\}\right) \mid \mathcal{F}_{t_{a}^{(m)}}\right] \leq\left[\sum_{r=2}^{\infty}\left\{\delta c_{1} \exp \left(-\delta c_{1}+v\right)\right\}^{r}\right]^{N M} .
$$

We take $m$ to be large enough that $\delta c_{1} \exp \left(-\delta c_{1}+v\right) \leq 1 / 4$, which means that the formula for summing a geometric sequence implies that

$$
\left[\sum_{r=2}^{\infty}\left\{\delta c_{1} \exp \left(-\delta c_{1}+v\right)\right\}^{r}\right]^{N M} \leq 4^{-N M}
$$


We then continue the argument, evaluating (139) conditionally on $\mathcal{F}_{t_{m-2}^{(m)}}$, then $\mathcal{F}_{t_{m-3}^{(m)}} \ldots$ and finally $\mathcal{F}_{t_{0}^{(m)}}$. We find that (139) must be less than or equal to $4^{-N M(m+1)} \exp \left(-\frac{N \in \mathfrak{v}}{20 C(\mathfrak{n})}\right)$. We have established (136).

We see that (137) is a difference between an integral and its time-discretized approximation, and can easily be shown to be true for large enough $m$.

\section{Taylor Expansion of Test Functions}

After the change of measure of the previous section, our task is easier, because now the spin-flipping intensity of $\tilde{\boldsymbol{\sigma}}_{i, t}$ is independent of the connections $\mathbf{J}$. This section (and the remainder of the paper) is oriented towards proving condition (124) of Lemma 4.6. This proof is accomplished through the comparison of the expectations of test functions, using the dual Kantorovich representation of the Wasserstein distance. We will Taylor expand the test functions to second order, and (in subsequent sections) demonstrate that the expectation with respect to the flow operator $\Psi_{t}$ almost matches the expectation with respect to the empirical process. Let $\mathfrak{H}$ be the set of all functions that are uniformly Lipschitz, i.e.

$$
\mathfrak{H}=\left\{f \in \mathcal{C}\left(\mathcal{E}^{M} \times \mathbb{R}^{M}\right):|f(\boldsymbol{\alpha}, \mathbf{x})-f(\boldsymbol{\beta}, \mathbf{z})| \leq\|\boldsymbol{\alpha}-\boldsymbol{\beta}\|+\|\mathbf{x}-\mathbf{z}\| \text { and } f(\mathbf{0})=0\right\} .
$$

It follows from the Kantorovich-Rubinstein theorem [34] that

$$
d_{W}(\mu, \nu)=\sup _{\mathbf{f} \in \mathfrak{H}}\left\{\left|\mathbb{E}^{\mu}[f]-\mathbb{E}^{\nu}[f]\right|\right\} .
$$

Our proofs only make use of a finite number of test functions: so we must demonstrate that the right hand side of the above equation can be approximated arbitrarily well by taking the supremum over a finite subset. Furthermore we require that the test functions are three-times differentiable in order that the expectations of stochastic fluctuations converge smoothly. Let $\mathfrak{H}_{a}$ be the set of all $f \in \mathfrak{H}$ satisfying the following assumptions.

- $f(\boldsymbol{\alpha}, \mathbf{x})=0$ for $\|\mathbf{x}\| \geq a$.

- $f(\boldsymbol{\alpha}, \mathbf{x})=\chi\{\boldsymbol{\alpha}=\boldsymbol{\beta}\} \bar{f}(\mathbf{x})$, for some fixed $\boldsymbol{\beta} \in \mathcal{E}^{M}$ and $\bar{f} \in \mathcal{C}^{3}\left(\mathbb{R}^{M}\right)$.

- Write the first, second and third order partial derivatives, in the second variable, as (respectively) $\bar{f}_{j}, \bar{f}_{j k}, \bar{f}_{j k l}$, for $j, k, l \in I_{M}$. These are all assumed to be uniformly bounded by 1 .

Lemma 5.1. For any $\delta>0$, there exists a $\in \mathbb{Z}^{+}$and a finite subset $\overline{\mathfrak{H}}_{a} \subset \mathfrak{H}_{a}$ such that for all $\mu, \nu \in \mathcal{W}_{2}$,

$$
d_{W}(\mu, \nu) \leq \delta+\sup _{f \in \tilde{\mathfrak{H}}_{a}}\left\{\left|\mathbb{E}^{\mu}[f]-\mathbb{E}^{\nu}[f]\right|\right\}
$$

Proof. For any $\mu \in \mathcal{W}_{2}$, any $f \in \mathfrak{H}$, and $a>0$,

$$
\begin{aligned}
\mathbb{E}^{\mu}[f \chi\{\|\mathbf{x}\| \geq a\}] & \leq \mathbb{E}^{\mu}[\|\mathbf{x}\| \chi\{\|\mathbf{x}\| \geq a\}] \\
& \leq a^{-1} \mathbb{E}^{\mu}\left[\|\mathbf{x}\|^{2}\right] \leq 3 / a
\end{aligned}
$$

Thus for any $\delta>0$, for large enough $a$,

$$
d_{W}(\mu, \nu) \leq \delta / 2+\sup _{\mathbf{f} \in \tilde{\mathfrak{H}}_{a}}\left\{\left|\mathbb{E}^{\mu}[f]-\mathbb{E}^{\nu}[f]\right|\right\}
$$

where $\tilde{\mathfrak{H}}_{a}$ is the set of all $f \in \mathfrak{H}$ such that $f(\boldsymbol{\alpha}, \mathbf{x})=0$ if $\|\mathbf{x}\| \geq a$. It remains to demonstrate that we can find a finite subset $\overline{\mathfrak{H}}_{a}$ of $\mathfrak{H}_{a}$ such that

$$
\sup _{\mathbf{f} \in \tilde{\mathfrak{H}}_{a}}\left\{\left|\mathbb{E}^{\mu}[f]-\mathbb{E}^{\nu}[f]\right|\right\} \leq \delta / 2+\sup _{\mathbf{f} \in \overline{\mathfrak{H}}_{a}}\left\{\left|\mathbb{E}^{\mu}[f]-\mathbb{E}^{\nu}[f]\right|\right\} .
$$


Since continuous functions on compact domains can be approximated arbitrarily well by smooth functions, it must be that

$$
\sup _{\mathbf{f} \in \tilde{\mathfrak{H}}_{a}}\left\{\left|\mathbb{E}^{\mu}[f]-\mathbb{E}^{\nu}[f]\right|\right\}=\sup _{\mathbf{f} \in \mathfrak{H}_{a}}\left\{\left|\mathbb{E}^{\mu}[f]-\mathbb{E}^{\nu}[f]\right|\right\}
$$

It follows from the Arzela-Ascoli Theorem that $\mathfrak{H}_{a}$ is compact. Thus we can find a finite cover of $\mathfrak{H}_{a}$ such that every function in $\mathfrak{H}_{a}$ is within $\delta / 2$ of a function in the finite cover (relative to the supremum norm).

Now set $\delta=\Delta \bar{\epsilon} / 2$, and let $a \in \mathbb{R}^{+}$and $\mathfrak{h}_{a}$ be such that for all $\mu, \nu \in \overline{\mathcal{P}}, d_{W}(\mu, \nu) \leq \delta+\sup _{\mathbf{f} \in \overline{\mathfrak{H}}_{a}}\left\{\mid \mathbb{E}^{\mu}[f]-\right.$ $\left.\mathbb{E}^{\nu}[f] \mid\right\}$. We write $\mathfrak{F} \subset \mathcal{C}^{3}\left(\mathbb{R}^{M}\right)$ to be such that

$$
\overline{\mathfrak{H}}_{a}=\left\{f(\boldsymbol{\alpha}, \mathbf{x})=\chi\{\boldsymbol{\alpha}=\boldsymbol{\beta}\} \phi(\mathbf{x}) \text { for some } \boldsymbol{\beta} \in \mathcal{E}^{M} \text { and } \phi \in \mathfrak{F}\right\}
$$

and we define the pseudo-metric ${ }^{5}$

$$
d_{K}(\mu, \nu)=\sup _{\phi \in \mathfrak{F}, \boldsymbol{\beta} \in \mathcal{E}^{M}}\left\{\left|\mathbb{E}^{\mu}[\phi(\mathbf{x}) \chi\{\boldsymbol{\alpha}=\boldsymbol{\beta}\}]-\mathbb{E}^{\nu}[\phi(\mathbf{x}) \chi\{\boldsymbol{\alpha}=\boldsymbol{\beta}\}]\right|\right\} .
$$

Henceforth we drop the subscript $\mathfrak{q}$ from the processes $\tilde{\boldsymbol{\sigma}}_{\mathfrak{q}, t}$ and $\tilde{\mathbf{G}}_{\mathfrak{q}, t}$. We find that for the condition (124) of Lemma 4.6 to be satisfied, it suffices for us to prove that for any $\bar{\epsilon}>0$, for all sufficiently large $n$ and all $0 \leq b \leq n-1$,

$$
\varlimsup_{N \rightarrow \infty} \sup _{1 \leq \mathfrak{q} \leq C_{\mathbf{n}}^{N}} N^{-1} \log \mathbb{P}\left(\mathcal{J}_{N}, \tilde{\mu}^{N}(\tilde{\boldsymbol{\sigma}}, \tilde{\mathbf{G}}) \in \mathcal{V}_{\mathfrak{q}}^{N}, \tilde{\tau}_{N}>t_{b}^{(n)}, d_{K}\left(\xi_{b}(\tilde{\boldsymbol{\sigma}}, \tilde{\mathbf{G}}), \hat{\mu}^{N}\left(\tilde{\boldsymbol{\sigma}}_{b+1}, \tilde{\mathbf{G}}_{b+1}\right)\right) \geq \bar{\epsilon} \Delta\right)<0
$$

recalling that $\tilde{G}_{t}^{p, j}=N^{-1 / 2} \sum_{k \in I_{N}} J^{j k} \tilde{\sigma}_{t}^{p, k}$ and $\xi_{b}(\tilde{\boldsymbol{\sigma}}, \tilde{\mathbf{G}})$ is the law of the random variables in (53)-(54). We emphasize that throughout the rest of this paper, $\tilde{\boldsymbol{\sigma}}_{b}:=\tilde{\boldsymbol{\sigma}}_{t_{b}^{(n)}}$ : that is the subscript is with respect to the $n+1$-point time discretization. Write the first derivative of $\phi \in \mathfrak{F}_{\mathfrak{m}}$ with respect to the $j^{\text {th }}$ variable as $\phi_{j}$, the second derivative of $\phi \in \mathfrak{F}_{\mathfrak{m}}$ with respect to the $j^{t h}$ and $k^{t h}$ variables as $\phi_{j k}$, and the third derivative as $\phi_{j k l}$.

We enumerate $\mathfrak{F}$ as $\mathfrak{F}=\left\{\phi^{a}\right\}_{a=1}^{|\mathfrak{F}|}$. For $\boldsymbol{\alpha} \in \mathcal{E}^{M}$, define

$$
\begin{aligned}
Q_{b}^{a, \boldsymbol{\alpha}} & =\mathbb{E}^{\hat{\mu}^{N}\left(\tilde{\boldsymbol{\sigma}}_{b+1}, \tilde{\mathbf{G}}_{b+1}\right)}\left[\phi^{a}(\mathbf{x}) \chi(\boldsymbol{\sigma}=\boldsymbol{\alpha})\right]=N^{-1} \sum_{j \in I_{N}} \chi\left\{\tilde{\boldsymbol{\sigma}}_{b+1}^{j}=\boldsymbol{\alpha}\right\} \phi^{a}\left(\tilde{\mathbf{G}}_{b+1}^{j}\right) \\
R_{b}^{a, \boldsymbol{\alpha}} & =\mathbb{E}^{\xi_{b+1}(\tilde{\boldsymbol{\sigma}}, \tilde{\mathbf{G}})}\left[\phi^{a}(\mathbf{x}) \chi(\boldsymbol{\sigma}=\boldsymbol{\alpha})\right] .
\end{aligned}
$$

We first establish a more workable expression for $R_{b}^{a, \boldsymbol{\alpha}}$.

Lemma 5.2. Recall that $\boldsymbol{\alpha}[i] \in \mathcal{E}^{M}$ is the same as $\boldsymbol{\alpha}$, except that the $i^{\text {th }}$ spin has a flipped sign.

$$
\begin{array}{r}
R_{b}^{a, \boldsymbol{\alpha}}=\Delta N^{-1} \sum_{j \in I_{N}, i \in I_{M}} \phi^{a}\left(\tilde{\mathbf{G}}_{b}^{j}\right) \chi\left\{\tilde{\boldsymbol{\sigma}}_{b}^{j}=\boldsymbol{\alpha}[i]\right\} c\left(-\alpha^{i}, \tilde{G}_{b}^{i, j}\right)+N^{-1} \sum_{j \in I_{N}} \chi\left\{\tilde{\boldsymbol{\sigma}}_{b}^{j}=\boldsymbol{\alpha}\right\}\left[\phi^{a}\left(\tilde{\mathbf{G}}_{b}^{j}\right)+\Delta \sum_{i \in I_{M}}\{\right. \\
\left.\left.\phi_{i}^{a}\left(\tilde{\mathbf{G}}_{b}^{j}\right) m^{\hat{\mu}_{b}^{N}, i}\left(\boldsymbol{\alpha}, \tilde{\mathbf{G}}_{b}^{j}\right)+2 L_{i i}^{\hat{\mu}_{b}^{N}} \phi_{i i}^{a}\left(\tilde{\mathbf{G}}_{b}^{j}\right)-\phi^{a}\left(\tilde{\mathbf{G}}_{b}^{j}\right) c\left(\alpha^{i}, \tilde{G}_{b}^{i, j}\right)\right\}\right]+O\left((\Delta)^{3 / 2}\right) .
\end{array}
$$

Proof. Recall the definition of $\xi_{b}(\tilde{\boldsymbol{\sigma}}, \tilde{\mathbf{G}})$, in terms of independent unit-intensity Poisson processes $\left\{\tilde{Y}^{p}(t)\right\}_{p \in I_{M}}$ and independent Brownian motions $\left\{\tilde{W}_{t}^{p}\right\}_{p \in I_{M}}$ in (53)-(54). We can then write $R_{b}^{a, \boldsymbol{\alpha}}$ as

$$
\begin{aligned}
R_{b}^{a, \boldsymbol{\alpha}} & =N^{-1} \sum_{j \in I_{N}} \mathbb{E}\left[\chi\left\{\boldsymbol{\zeta}_{\Delta}^{j}=\boldsymbol{\alpha}\right\} \phi^{a}\left(\mathbf{x}_{\Delta}^{j}\right) \mid \tilde{\boldsymbol{\sigma}}_{b}, \tilde{\mathbf{G}}_{b}\right] \text { where } \boldsymbol{\zeta}_{\Delta}^{j}=\left(\zeta_{\Delta}^{p, j}\right)_{p \in I_{M}} \text { and } \\
\zeta_{\Delta}^{p, j} & =\tilde{\sigma}_{b}^{p, j} A \cdot \tilde{Y}^{p}\left(\Delta c\left(\tilde{\sigma}_{b}^{p, j}, \tilde{G}_{b}^{p, j}\right)\right) \\
\mathbf{x}_{\Delta}^{j} & =\tilde{\mathbf{G}}_{b}^{j}+\Delta \mathbf{m}^{\hat{\mu}_{b}^{N}}\left(\tilde{\boldsymbol{\sigma}}_{b}^{j}, \tilde{\mathbf{G}}_{b}^{j}\right)+\mathbf{D}^{\hat{\mu}_{b}^{N}} \tilde{\mathbf{W}}_{\Delta} \text { where } D_{i j}^{\hat{\mu}_{b}^{N}}=2 \sqrt{L_{i i}^{\hat{\mu}_{b}^{N}}} \delta(i, j),
\end{aligned}
$$

\footnotetext{
${ }^{5}$ This satisfies all of the axioms of a metric, except that $d_{K}(\mu, \nu)=0$ does not necessarily imply that $\mu=\nu$.
} 
and the expectation in (151) is taken with respect to the $\tilde{\mathbf{Y}}$ and $\tilde{\mathbf{W}}_{\Delta}$ random variables, holding $\tilde{\boldsymbol{\sigma}}_{b}$ and $\tilde{\mathbf{G}}_{b}$ to be fixed, and $A \cdot x:=(-1)^{x}$. Write

$$
X_{b+1}^{j}=\chi\left\{\boldsymbol{\zeta}_{\Delta}^{j}=\boldsymbol{\alpha}\right\}-\chi\left\{\tilde{\boldsymbol{\sigma}}_{b}^{j}=\boldsymbol{\alpha}\right\}+\Delta \sum_{i \in I_{M}}\left\{c\left(\tilde{\sigma}_{b}^{i, j}, \tilde{G}_{b}^{i, j}\right) \chi\left\{\tilde{\boldsymbol{\sigma}}_{b}^{j}=\boldsymbol{\alpha}\right\}-c\left(-\tilde{\sigma}_{b}^{i, j}, \tilde{G}_{b}^{i, j}\right) \chi\left\{\tilde{\boldsymbol{\sigma}}_{b}^{j}=\boldsymbol{\alpha}[i]\right\}\right\} .
$$

Basic properties of the Poisson process - and recalling that the jump intensity $c$ is uniformly upperbounded by $c_{1}$ - imply that $\mathbb{E}\left[X_{b+1}^{j} \mid \tilde{\boldsymbol{\sigma}}_{b}, \tilde{\mathbf{G}}_{b}\right]=O\left(\Delta^{2}\right)[27]$ (one can see from the Komolgorov Forward equation (18) why this is true). We thus find that

$$
\begin{array}{r}
R_{b}^{a, \boldsymbol{\alpha}}=N^{-1} \sum_{j \in I_{N}}\left(\chi\left\{\tilde{\boldsymbol{\sigma}}_{b}^{j}=\boldsymbol{\alpha}\right\}+\Delta \sum_{i \in I_{M}}\left\{c\left(-\tilde{\sigma}_{b}^{i, j}, \tilde{G}_{b}^{i, j}\right) \chi\left\{\tilde{\boldsymbol{\sigma}}_{b}^{j}=\boldsymbol{\alpha}[i]\right\}-c\left(\tilde{\sigma}_{b}^{i, j}, \tilde{G}_{b}^{i, j}\right) \chi\left\{\tilde{\boldsymbol{\sigma}}_{b}^{j}=\boldsymbol{\alpha}\right\}\right\}\right) \\
\times \mathbb{E}\left[\phi^{a}\left(\tilde{\mathbf{G}}_{b}^{j}+\Delta \mathbf{m}^{\hat{\mu}_{b}^{N}}\left(\tilde{\boldsymbol{\sigma}}_{b}^{j}, \tilde{\mathbf{G}}_{b}^{j}\right)+\mathbf{D}^{\hat{\mu}_{b}^{N}} \tilde{\mathbf{W}}_{\Delta}\right) \mid \tilde{\boldsymbol{\sigma}}_{b}, \tilde{\mathbf{G}}_{b}\right]+O\left(\Delta^{2}\right) .
\end{array}
$$

Applying a Taylor expansion, and noting that the third order partial derivatives of $\phi^{a}$ are uniformly bounded, we obtain that

$$
\begin{aligned}
& \mathbb{E}\left[\phi^{a}\left(\tilde{\mathbf{G}}_{b}^{j}+\Delta \mathbf{m}^{\hat{\mu}_{b}^{N}}\left(\tilde{\boldsymbol{\sigma}}_{b}^{j}, \tilde{\mathbf{G}}_{b}^{j}\right)+\mathbf{D}^{\hat{\mu}_{b}^{N}} \tilde{\mathbf{W}}_{\Delta}\right) \mid \tilde{\boldsymbol{\sigma}}, \tilde{\mathbf{G}}\right] \\
&=\phi^{a}\left(\tilde{\mathbf{G}}_{b}^{j}\right)+\Delta \sum_{i \in I_{M}}\left\{\phi_{i}^{a}\left(\tilde{\mathbf{G}}_{b}^{j}\right) m^{\hat{\mu}_{b}^{N}, i}\left(\tilde{\boldsymbol{\sigma}}_{b}^{j}, \tilde{\mathbf{G}}_{b}^{j}\right)+\left(D_{i i}^{\hat{\mu}_{b}^{N}}\right)^{2} \phi_{i i}^{a}\left(\tilde{\mathbf{G}}_{b}^{j}\right) / 2\right\}+O\left(\Delta^{3 / 2}\right),
\end{aligned}
$$

since $\mathbb{E}\left[\left\|\tilde{\mathbf{W}}_{\Delta}\right\|^{3}\right]=O\left((\Delta)^{3 / 2}\right)$. This implies the lemma.

Using a union-of-events bound, we obtain that

$$
\begin{aligned}
\mathbb{P}\left(\mathcal{J}_{N}, \tilde{\mu}^{N} \in \mathcal{V}_{\mathfrak{q}}^{N}, d_{K}\left(\xi_{b}(\tilde{\boldsymbol{\sigma}}, \tilde{\mathbf{G}}), \hat{\mu}^{N}\left(\tilde{\boldsymbol{\sigma}}_{b+1}, \tilde{\mathbf{G}}_{b+1}\right)\right) \geq \bar{\epsilon} \Delta\right) & \\
& \leq \sum_{\phi^{a} \in \mathfrak{F} \boldsymbol{\alpha} \in \mathcal{E}^{M}} \sum \mathbb{P}\left(\mathcal{J}_{N}, \tilde{\mu}^{N} \in \mathcal{V}_{\mathfrak{q}}^{N},\left|Q_{b}^{a, \boldsymbol{\alpha}}-R_{b}^{a, \boldsymbol{\alpha}}\right| \geq \bar{\epsilon} \Delta\right) .
\end{aligned}
$$

The implication of the above argument is that, in order that (147) is satisfied, and making use of Lemma 3.1 , it suffices to prove the following lemma.

Lemma 5.3. In order that condition (124) of Lemma 4.6 is satisfied, it suffices to prove the following statement. For any $\epsilon>0$, there exists $n_{0}$ such that for all $n \geq n_{0}$, there exists $\mathfrak{n}_{0} \in \mathbb{Z}^{+}$such that for all $\mathfrak{n} \geq \mathfrak{n}_{0}$,

$$
\sup _{0 \leq b \leq n-1} \varlimsup_{N \rightarrow \infty} \sup _{1 \leq \mathfrak{q} \leq C_{\mathbf{n}}^{N}} \sup _{\phi^{a} \in \mathfrak{F}, \boldsymbol{\alpha} \in \mathcal{E}^{M}} N^{-1} \log \mathbb{P}\left(\mathcal{J}_{N}, \tilde{\mu}^{N}(\tilde{\boldsymbol{\sigma}}, \tilde{\mathbf{G}}) \in \mathcal{V}_{\mathfrak{q}}^{N}, \tilde{\tau}_{N}>t_{b}^{(n)},\left|Q_{b}^{a, \boldsymbol{\alpha}}-R_{b}^{a, \boldsymbol{\alpha}}\right| \geq \epsilon \Delta\right)<0 .
$$

Substituting the expression for $R_{b+1}^{a, \boldsymbol{\alpha}}$ in Lemma 5.2, we find that the difference can be decomposed as

$$
\sum_{\boldsymbol{\alpha} \in \mathcal{E}^{M}}\left(Q_{b+1}^{a, \boldsymbol{\alpha}}-R_{b+1}^{a, \boldsymbol{\alpha}}\right)=O\left(\Delta^{3 / 2}\right)+\sum_{\boldsymbol{\alpha} \in \mathcal{E}^{M}} \sum_{i=1}^{6} \beta^{i}(\boldsymbol{\alpha}, \tilde{\boldsymbol{\sigma}}, \tilde{\mathbf{G}})
$$

and $\left\{\beta^{i}\right\}_{i=1}^{5}$ are defined as follows (the dependence of $\beta^{i}$ on $a$ has been neglected from the notation). Our aim is to decompose the difference into terms that can either be controlled with Poisson concentration inequalities or controlled with the Gaussian law of the connections. Here and below, $\hat{\mu}_{b}^{N}:=\hat{\mu}_{b}^{N}(\tilde{\boldsymbol{\sigma}}, \tilde{\mathbf{G}})$. The term $\beta^{1}$ represents the leading order change in the two expectations due to jumps in the spins, while holding the field to be constant, i.e.

$$
\begin{aligned}
\beta^{1}(\boldsymbol{\alpha}, \tilde{\boldsymbol{\sigma}}, \tilde{\mathbf{G}})=N^{-1} \sum_{j \in I_{N}} \phi^{a}\left(\tilde{\mathbf{G}}_{b}^{j}\right)\left(\chi \left\{\tilde{\boldsymbol{\sigma}}_{b+1}^{j}\right.\right. & =\boldsymbol{\alpha}\}-\chi\left\{\tilde{\boldsymbol{\sigma}}_{b}^{j}=\boldsymbol{\alpha}\right\} \\
& \left.-\Delta \sum_{i \in I_{M}}\left\{c\left(-\alpha^{i}, \tilde{G}_{b}^{i, j}\right) \chi\left\{\tilde{\boldsymbol{\sigma}}_{b}^{j}=\boldsymbol{\alpha}[i]\right\}-c\left(\alpha^{i}, \tilde{G}_{b}^{i, j}\right) \chi\left\{\tilde{\boldsymbol{\sigma}}_{b}^{j}=\boldsymbol{\alpha}\right\}\right\}\right),
\end{aligned}
$$


recalling that $\boldsymbol{\alpha}[i]$ is the same as $\boldsymbol{\alpha}$, except that the $i^{\text {th }}$ element has a flipped sign.

The sum of the terms $\beta^{2}+\beta^{3}$ represents the leading order change in the two expectations due to changes in the field $\tilde{\mathbf{G}}_{t}$, while holding the spin to be constant. A Taylor approximation is used: $\beta^{2}$ contains the linear terms, and $\beta^{3}$ the quadratic terms,

$$
\begin{aligned}
& \beta^{2}(\boldsymbol{\alpha}, \tilde{\boldsymbol{\sigma}}, \tilde{\mathbf{G}})=N^{-1} \sum_{j \in I_{N}} \sum_{i \in I_{M}} \phi_{i}^{a}\left(\tilde{\mathbf{G}}_{b}^{j}\right) \chi\left\{\tilde{\boldsymbol{\sigma}}_{b}^{j}=\boldsymbol{\alpha}\right\}\left\{\tilde{G}_{b+1}^{i, j}-\tilde{G}_{b}^{i, j}-\Delta m^{\hat{\mu}_{b}^{N}, i}\left(\boldsymbol{\alpha}, \tilde{\mathbf{G}}_{b}^{j}\right)\right\} \\
& \beta^{3}(\boldsymbol{\alpha}, \tilde{\boldsymbol{\sigma}}, \tilde{\mathbf{G}})=(2 N)^{-1} \sum_{j \in I_{N}} \sum_{i, p \in I_{M}} \chi\left\{\tilde{\boldsymbol{\sigma}}_{b}^{j}=\boldsymbol{\alpha}\right\}\left(\phi_{i p}^{a}\left(\tilde{\mathbf{G}}_{b}^{j}\right)\left\{\tilde{G}_{b+1}^{i, j}-\tilde{G}_{b}^{i, j}\right\}\left\{\tilde{G}_{b+1}^{p, j}-\tilde{G}_{b}^{p, j}\right\}-4 L_{i i}^{\hat{\mu}_{b}^{N}} \Delta\right) .
\end{aligned}
$$

$\beta^{4}$ can be thought of as the average 'cross-variation' between the spins and the fields:

$$
\beta^{4}(\boldsymbol{\alpha}, \tilde{\boldsymbol{\sigma}}, \tilde{\mathbf{G}})=N^{-1} \sum_{j \in I_{N}}\left\{\phi^{a}\left(\tilde{\mathbf{G}}_{b+1}^{j}\right)-\phi^{a}\left(\tilde{\mathbf{G}}_{b}^{j}\right)\right\}\left\{\chi\left\{\tilde{\boldsymbol{\sigma}}_{b+1}^{j}=\boldsymbol{\alpha}\right\}-\chi\left\{\tilde{\boldsymbol{\sigma}}_{b}^{j}=\boldsymbol{\alpha}\right\}\right\} .
$$

The term $\beta^{5}$ is the remainder, such that (156) holds identically. This means that

$$
\begin{aligned}
\beta^{5}(\boldsymbol{\alpha}, \tilde{\boldsymbol{\sigma}}, \tilde{\mathbf{G}})=N^{-1} \sum_{j \in I_{N}} \chi\left\{\tilde{\boldsymbol{\sigma}}_{b}^{j}=\boldsymbol{\alpha}\right\}\left(\phi^{a}\left(\tilde{\mathbf{G}}_{b+1}^{j}\right)-\phi^{a}\left(\tilde{\mathbf{G}}_{b}^{j}\right)-\sum_{i \in I_{M}} \phi_{i}^{a}\left(\tilde{\mathbf{G}}_{b}^{j}\right)\left\{\tilde{G}_{b+1}^{i, j}-\tilde{G}_{b}^{i, j}\right\}\right. \\
\left.-\frac{1}{2} \sum_{i, p \in I_{M}} \phi_{i p}^{a}\left(\tilde{\mathbf{G}}_{b}^{j}\right)\left\{\tilde{G}_{b+1}^{i, j}-\tilde{G}_{b}^{i, j}\right\}\left\{\tilde{G}_{b+1}^{p, j}-\tilde{G}_{b}^{p, j}\right\}\right) .
\end{aligned}
$$

We further decompose $\beta^{2}$ and $\beta^{3}$ as follows. The terms $\beta^{6}, \beta^{8}$ and $\beta^{9}$ - to be outlined just below - will be bounded in Section 7 using the conditional Gaussian law of the connections. The term $\tilde{\mathbf{m}}_{b}^{j}$ - to be outlined just below - is the mean of a conditional Gaussian expectation, and $\tilde{\mathbf{L}}_{b}^{i j}$ is approximately half the conditional variance. Define the $M \times M$ matrices $\left\{\tilde{\mathbf{K}}_{b}, \tilde{\mathbf{L}}_{b}, \tilde{\boldsymbol{\kappa}}_{b}, \tilde{\boldsymbol{v}}_{b}\right\}$ to have the following elements: for $p, q \in I_{M}$

$$
\begin{aligned}
& \tilde{K}_{b}^{p q}=N^{-1} \sum_{l=1}^{N} \tilde{\sigma}_{b}^{p, l} \tilde{\sigma}_{b}^{q, l}, \tilde{L}_{b}^{p q}=N^{-1} \sum_{k=1}^{N} \tilde{\sigma}_{b}^{q, k}\left(\tilde{\sigma}_{b}^{p, k}-\tilde{\sigma}_{b+1}^{p, k}\right) \\
& \tilde{\kappa}_{b}^{p q}=N^{-1} \sum_{k=1}^{N} \tilde{G}_{b}^{q, k}\left(\tilde{\sigma}_{b}^{p, k}-\tilde{\sigma}_{b+1}^{p, k}\right), \tilde{v}_{b}^{p q}=N^{-1} \sum_{k=1}^{N} \tilde{\sigma}_{b}^{p, k} \tilde{G}_{b}^{q, k} .
\end{aligned}
$$

If $\tilde{\tau}_{N}>t_{b}^{(n)}, \tilde{\mathbf{K}}_{b}$ is invertible, and we write $\tilde{\mathbf{H}}_{b}=\tilde{\mathbf{K}}_{b}^{-1}$. For $j \in I_{N}$, writing $\tilde{\boldsymbol{\sigma}}_{b}^{j}=\left(\sigma_{b}^{1, j}, \ldots, \sigma_{b}^{M, j}\right), \tilde{\mathbf{G}}_{b}^{j}=$ $\left(\tilde{G}_{b}^{1, j}, \ldots, \tilde{G}_{b}^{M, j}\right)$ and $\tilde{\mathbf{m}}_{b}^{j}=\left(\tilde{m}_{b}^{1, j}, \ldots, \tilde{m}_{b}^{M, j}\right)$, we define

$$
\tilde{\mathbf{m}}_{b}^{j}=-\tilde{\mathbf{L}}_{b} \tilde{\mathbf{H}}_{b} \tilde{\mathbf{G}}_{b}^{j}-\mathfrak{s} \tilde{\boldsymbol{\kappa}}_{b} \tilde{\mathbf{H}}_{b} \tilde{\boldsymbol{\sigma}}_{b}^{j}+\mathfrak{s} \tilde{\mathbf{L}}_{b} \tilde{\mathbf{H}}_{b} \tilde{\boldsymbol{v}}_{b} \tilde{\mathbf{H}}_{b} \tilde{\boldsymbol{\sigma}}_{b}^{j} .
$$

We can now further decompose $\beta^{2}$ as follows,

$$
\begin{aligned}
& \beta^{2}(\boldsymbol{\alpha}, \tilde{\boldsymbol{\sigma}}, \tilde{\mathbf{G}})=\beta^{6}(\boldsymbol{\alpha}, \tilde{\boldsymbol{\sigma}}, \tilde{\mathbf{G}})+\beta^{7}(\boldsymbol{\alpha}, \tilde{\boldsymbol{\sigma}}, \tilde{\mathbf{G}}) \text { where } \\
& \beta^{6}(\boldsymbol{\alpha}, \tilde{\boldsymbol{\sigma}}, \tilde{\mathbf{G}})=N^{-1} \sum_{j \in I_{N}} \sum_{i \in I_{M}} \phi_{i}^{a}\left(\tilde{\mathbf{G}}_{b}^{j}\right) \chi\left\{\tilde{\boldsymbol{\sigma}}_{b}^{j}=\boldsymbol{\alpha}\right\}\left\{\tilde{G}_{b+1}^{i, j}-\tilde{G}_{b}^{i, j}-\tilde{m}_{b}^{i, j}\right\} \\
& \beta^{7}(\boldsymbol{\alpha}, \tilde{\boldsymbol{\sigma}}, \tilde{\mathbf{G}})=N^{-1} \sum_{j \in I_{N}} \sum_{i \in I_{M}} \phi_{i}^{a}\left(\tilde{\mathbf{G}}_{b}^{j}\right) \chi\left\{\tilde{\boldsymbol{\sigma}}_{b}^{j}=\boldsymbol{\alpha}\right\}\left\{\tilde{m}_{b}^{i, j}-\Delta m^{\hat{\mu}_{b}^{N}, i}\left(\tilde{\boldsymbol{\sigma}}_{b}^{j}, \tilde{\mathbf{G}}_{b}^{j}\right)\right\},
\end{aligned}
$$


noting that $m^{\hat{\mu}_{b}^{N}}$ is defined in (30). We further decompose $\beta^{3}(\boldsymbol{\alpha}, \tilde{\boldsymbol{\sigma}}, \tilde{\mathbf{G}})$ as follows

$$
\begin{aligned}
\beta^{3}(\boldsymbol{\alpha}, \tilde{\boldsymbol{\sigma}}, \tilde{\mathbf{G}})= & \beta^{8}(\boldsymbol{\alpha}, \tilde{\boldsymbol{\sigma}}, \tilde{\mathbf{G}})+\beta^{9}(\boldsymbol{\alpha}, \tilde{\boldsymbol{\sigma}}, \tilde{\mathbf{G}})+\beta^{10}\left(\boldsymbol{\alpha}, \tilde{\boldsymbol{\sigma}}_{b}, \tilde{\mathbf{G}}_{b}\right)+\beta^{11}\left(\boldsymbol{\alpha}, \tilde{\boldsymbol{\sigma}}_{b}, \tilde{\mathbf{G}}_{b}\right) \text { where } \\
\beta^{8}(\boldsymbol{\alpha}, \tilde{\boldsymbol{\sigma}}, \tilde{\mathbf{G}})= & N^{-1} \sum_{j \in I_{N}} \sum_{i, p \in I_{M}} \chi\left\{\tilde{\boldsymbol{\sigma}}_{b}^{j}=\boldsymbol{\alpha}\right\} \phi_{i p}^{a}\left(\tilde{\mathbf{G}}_{b}^{j}\right) \tilde{m}_{b}^{i, j}\left\{\tilde{G}_{b+1}^{p, j}-\tilde{G}_{b}^{p, j}-\tilde{m}_{b}^{p, j}\right\} \\
\beta^{9}(\boldsymbol{\alpha}, \tilde{\boldsymbol{\sigma}}, \tilde{\mathbf{G}})= & (2 N)^{-1} \sum_{j \in I_{N}} \sum_{i, p \in I_{M}} \chi\left\{\tilde{\boldsymbol{\sigma}}_{b}^{j}=\boldsymbol{\alpha}\right\} \phi_{i p}^{a}\left(\tilde{\mathbf{G}}_{b}^{j}\right)\left\{\tilde{G}_{b+1}^{i, j}-\tilde{G}_{b}^{i, j}-\tilde{m}_{b}^{i, j}\right\}\left\{\tilde{G}_{b+1}^{p, j}-\tilde{G}_{b}^{p, j}-\tilde{m}_{b}^{p, j}\right\} \\
& -(N)^{-1} \sum_{j \in I_{N}} \sum_{i \in I_{M}} \chi\left\{\tilde{\boldsymbol{\sigma}}_{b}^{j}=\boldsymbol{\alpha}\right\} \phi_{i i}^{a}\left(\tilde{\mathbf{G}}_{b}^{j}\right) \tilde{L}_{b}^{i i} \\
\beta^{10}(\boldsymbol{\alpha}, \tilde{\boldsymbol{\sigma}}, \tilde{\mathbf{G}})= & (2 N)^{-1} \sum_{j \in I_{N}} \sum_{i, p \in I_{M}} \chi\left\{\tilde{\boldsymbol{\sigma}}_{b}^{j}=\boldsymbol{\alpha}\right\} \phi_{i p}^{a}\left(\tilde{\mathbf{G}}_{b}^{j}\right) \tilde{m}_{b}^{i, j} \tilde{m}_{b}^{p, j} \\
\beta^{11}(\boldsymbol{\alpha}, \tilde{\boldsymbol{\sigma}}, \tilde{\mathbf{G}})= & N^{-1} \sum_{j \in I_{N}} \sum_{i \in I_{M}} \chi\left\{\tilde{\boldsymbol{\sigma}}_{b}^{j}=\boldsymbol{\alpha}\right\} \phi_{i i}^{a}\left(\tilde{\mathbf{G}}_{b}^{j}\right)\left(\tilde{L}_{b}^{i i}-2 \Delta L_{i i}^{\hat{\mu}_{b}^{N}}\right) .
\end{aligned}
$$

We can now decompose the criteria of Lemma 5.3 into the following set of criteria.

Lemma 5.4. To prove Lemma 4.6 it suffices for us to show that for any $\bar{\epsilon}>0$, there exists $n_{0}$ such that for all $n \geq n_{0}$, there exists $\mathfrak{n}_{0} \in \mathbb{Z}^{+}$such that for all $\mathfrak{n} \geq \mathfrak{n}_{0}$, for each $i$ such that $1 \leq i \leq 11$ (with $i \neq 2,3$ ), (recalling that $\Delta=T n^{-1}$ )

$$
\sup _{0 \leq b<n} \sup _{\phi^{a} \in \widetilde{F}, \boldsymbol{\alpha} \in \mathcal{E}^{M}} \varlimsup_{N \rightarrow \infty} N^{-1} \log \sup _{1 \leq \mathfrak{q} \leq C_{\mathfrak{n}}^{N}} \mathbb{P}\left(\mathcal{J}_{N}, \tilde{\mu}^{N}(\tilde{\boldsymbol{\sigma}}, \tilde{\mathbf{G}}) \in \mathcal{V}_{\mathfrak{q}}^{N}, \tilde{\tau}_{N}>t_{b}^{(n)},\left|\beta^{i}(\boldsymbol{\alpha}, \tilde{\boldsymbol{\sigma}}, \tilde{\mathbf{G}})\right| \geq \bar{\epsilon} \Delta / 18\right)<0 .
$$

Proof. The above analysis implies that for large enough $n$,

$$
\begin{aligned}
\left\{d_{K}\left(\hat{\mu}^{N}\left(\tilde{\boldsymbol{\sigma}}_{b+1}, \tilde{\mathbf{G}}_{b+1}\right), \xi\left(\tilde{\boldsymbol{\sigma}}_{b}, \tilde{\mathbf{G}}_{b}\right)\right) \geq \bar{\epsilon} \Delta\right\} \subseteq \bigcup_{1 \leq i \leq 11, i \neq 2,3}\left\{\left|\beta^{i}(\boldsymbol{\alpha}, \tilde{\boldsymbol{\sigma}}, \tilde{\mathbf{G}})\right| \geq \bar{\epsilon} \Delta / 18\right\} \\
\cup\left\{\mid \Delta N^{-1} \sum_{j \in I_{N}, i \in I_{M}} \phi^{a}\left(\tilde{\mathbf{G}}_{b}^{j}\right) \chi\left\{\tilde{\boldsymbol{\sigma}}_{b}^{j}=\boldsymbol{\alpha}[i]\right\} c\left(-\alpha^{i}, \tilde{G}_{b}^{i, j}\right)+N^{-1} \sum_{j \in I_{N}} \chi\left\{\tilde{\boldsymbol{\sigma}}_{b}^{j}=\boldsymbol{\alpha}\right\}\left[\phi^{a}\left(\tilde{\boldsymbol{\sigma}}_{b}^{j}\right)+\Delta \sum_{i \in I_{M}}\{\right.\right. \\
\left.\left.\left.\phi_{i}^{a}\left(\tilde{\mathbf{G}}_{b}^{j}\right) m^{\hat{\mu}_{b}^{N}, i}\left(\boldsymbol{\alpha}, \tilde{\mathbf{G}}_{b}^{j}\right)+2 L_{i i}^{\hat{\mu}_{b}^{N}} \phi_{i i}^{a}\left(\tilde{\mathbf{G}}_{b}^{j}\right)-\phi^{a}\left(\tilde{\boldsymbol{\sigma}}_{b}^{j}\right) c\left(\alpha^{i}, \tilde{G}_{b}^{i, j}\right)\right\}\right]-R_{b}^{a, \boldsymbol{\alpha}} \mid \geq \bar{\epsilon} \Delta / 2\right\} .
\end{aligned}
$$

By Lemma 5.2, as long as $\Delta$ is sufficiently small,

$$
\begin{aligned}
\left\{\mid \Delta N^{-1} \sum_{j \in I_{N}, i \in I_{M}} \phi^{a}\left(\tilde{\mathbf{G}}_{b}^{j}\right) \chi\left\{\tilde{\boldsymbol{\sigma}}_{b}^{j}=\boldsymbol{\alpha}[i]\right\} c\left(-\alpha^{i}, \tilde{G}_{b}^{i, j}\right)+N^{-1} \sum_{j \in I_{N}} \chi\left\{\tilde{\boldsymbol{\sigma}}_{b}^{j}=\boldsymbol{\alpha}\right\}\left[\phi^{a}\left(\tilde{\boldsymbol{\sigma}}_{b}^{j}\right)+\Delta \sum_{i \in I_{M}}\{\right.\right. \\
\left.\left.\left.\phi_{i}^{a}\left(\tilde{\mathbf{G}}_{b}^{j}\right) m^{\hat{\mu}_{b}^{N}, i}\left(\boldsymbol{\alpha}, \tilde{\mathbf{G}}_{b}^{j}\right)+2 L_{i i}^{\hat{\mu}_{b}^{N}} \phi_{i i}^{a}\left(\tilde{\mathbf{G}}_{b}^{j}\right)-\phi^{a}\left(\tilde{\boldsymbol{\sigma}}_{b}^{j}\right) c\left(\alpha^{i}, \tilde{G}_{b}^{i, j}\right)\right\}\right]-R_{b}^{a, \boldsymbol{\alpha}} \mid \geq \bar{\epsilon} \Delta / 2\right\}=\varnothing
\end{aligned}
$$

The Lemma now follows as a consequence of Lemma 3.1, since $|\mathfrak{F}|<\infty$.

The nine bounds necessary for Lemma 5.4 are contained in the next two sections. They are split into two types: the terms directly requiring the law of the Gaussian connections (i.e. $\beta^{6}, \beta^{8}, \beta^{9}$ ) are bounded in Section 7. The other six terms mostly require concentration inequalities for Poisson processes, and they are bounded in Section 6 .

\section{Stochastic Bounds}

This section is devoted to bounding the terms in Lemma 5.4 that do not directly require the law of the Gaussian connections (i.e. $\gamma$ ). The terms that are bounded in the first part of this section are $\beta^{4}$ (the 'cross-variation' of the spins and fields) and $\beta^{5}$ (the remainder after the Taylor Expansion). In the next subsection, the remaining terms $\beta^{1}, \beta^{7}, \beta^{10}, \beta^{11}$ are bounded: the bounding of these terms requires concentration inequalities for sums of compensated Poisson Processes. Throughout this section we omit the $\mathfrak{q}$ subscript from the stochastic process, writing $\tilde{\boldsymbol{\sigma}}_{\mathfrak{q}, t}:=\tilde{\boldsymbol{\sigma}}_{t}$. 
Throughout this section $\boldsymbol{\alpha} \in \mathcal{E}^{M}$ is a fixed constant. Define for $u \geq 0$,

$$
Y_{b}^{i, j}(u)=Y^{i, j}\left(u+\int_{0}^{t_{b}^{(n)}} c\left(\tilde{\sigma}_{s}^{i, j}, \tilde{\mathfrak{G}}_{\mathfrak{q}, s}^{i, j}(\tilde{\boldsymbol{\sigma}})\right) d s\right)-Y^{i, j}\left(\int_{0}^{t_{b}^{(n)}} c\left(\tilde{\sigma}_{s}^{i, j}, \tilde{\mathfrak{G}}_{\mathfrak{q}, s}^{i, j}(\tilde{\boldsymbol{\sigma}})\right) d s\right),
$$

and notice that $\left\{Y_{b}^{i, j}(t)\right\}_{i \in I_{M}, j \in I_{N}}$ are distributed as iid unit intensity Poisson Processes. Recalling that $A \cdot x:=(-1)^{x}$, it may be inferred from the definition in (116) that for $t \geq t_{b}^{(n)}$,

$$
\tilde{\sigma}_{t}^{i, j}=\tilde{\sigma}_{b}^{i, j} A \cdot Y_{b}^{i, j}\left(\int_{t_{b}^{(n)}}^{t} c\left(\tilde{\sigma}_{s}^{i, j}, \tilde{\mathfrak{G}}_{\mathfrak{q}, s}^{i, j}\right) d s\right)
$$

Let $\mathcal{I}_{N}=\left\{j \in I_{N}:\right.$ For some $\left.i \in I_{M}, Y_{b}^{i, j}\left(c_{1} \Delta\right) \geq 1\right\}$, recalling that $c_{1}$ is the uniform upper bound for the spin flipping rate. Clearly if $j \notin \mathcal{I}_{N}$ then $\tilde{\boldsymbol{\sigma}}_{b}^{j}=\tilde{\boldsymbol{\sigma}}_{b+1}^{j}$. Write $\mathcal{I}_{N}^{c}=\left\{j \in I_{N}: j \notin \mathcal{I}_{N}\right\}$. Splitting the indices as $I_{N}=\mathcal{I}_{N} \cup \mathcal{I}_{N}^{c}$ is useful because the fields $\left\{\tilde{G}_{b+1}^{p, j}-\tilde{G}_{b}^{p, j}\right\}_{j \in \mathcal{I}_{N}^{c}}$ are independent.

We start with a lemma concerning the average change in fields indexed by $\mathcal{I}_{N}$.

Lemma 6.1. There exists $n_{0} \in \mathbb{Z}^{+}$and a constant $\hat{C}_{\gamma}$ such that for all $n \geq n_{0}$,

$$
\varlimsup_{N \rightarrow \infty} \sup _{1 \leq \mathfrak{q} \leq C_{\mathfrak{n}}^{N}} N^{-1} \log \mathbb{P}\left(N^{-1} \sum_{j \in \mathcal{I}_{N}}\left\|\tilde{\mathbf{G}}_{b+1}^{j}-\tilde{\mathbf{G}}_{b}^{j}\right\|^{2} \geq \Delta^{3 / 2} \hat{C}_{\gamma}\right)<0 .
$$

Proof. Let $\tilde{\mathbf{J}}_{N}$ be the $\left|\mathcal{I}_{N}\right| \times\left|\mathcal{I}_{N}\right|$ square matrix with entries given by $\left\{N^{-1 / 2} J^{j k}\right\}_{j, k \in \mathcal{I}_{N}}$. Let its operator norm be $\left\|\tilde{\mathbf{J}}_{N}\right\|$. Observe that

$$
\begin{aligned}
N^{-1} \sum_{j \in \mathcal{I}_{N}, i \in I_{M}}\left|\tilde{G}_{b+1}^{i, j}-\tilde{G}_{b}^{i, j}\right|^{2} & \leq N^{-1}\left\|\tilde{\mathbf{J}}_{N}\right\| \sum_{j \in \mathcal{I}_{N}, i \in I_{M}}\left|\tilde{\sigma}_{b+1}^{i, j}-\tilde{\sigma}_{b}^{i, j}\right|^{2} \\
& \leq 4 M N^{-1}\left\|\tilde{\mathbf{J}}_{N}\right\| \sum_{j \in \mathcal{I}_{N}} \chi\left\{\text { For some } i \in I_{M}, \tilde{\sigma}_{b+1}^{i, j} \neq \tilde{\sigma}_{b}^{i, j}\right\} .
\end{aligned}
$$

Writing $\hat{C}_{\gamma}=6 \sqrt{2} c_{1}^{3 / 2} M(M+1)$, we observe that if $\left\|\tilde{\mathbf{J}}_{N}\right\| \leq 3 \sqrt{c_{1} \Delta / 2}$ and $N^{-1} \sum_{j \in \mathcal{I}_{N}} \chi\{$ For some $i \in$ $\left.I_{M}, \tilde{\sigma}_{b+1}^{i, j} \neq \tilde{\sigma}_{b}^{i, j}\right\} \leq c_{1} \Delta(M+1)$ then $N^{-1} \sum_{j \in \mathcal{I}_{N}}\left\|\tilde{G}_{b+1}^{i, j}-\tilde{G}_{b}^{i, j}\right\|^{2} \leq \hat{C}_{\gamma} \Delta^{3 / 2}$. We thus find that,

$$
\begin{aligned}
&\left\{N^{-1} \sum_{j \in \mathcal{I}_{N}}\left\|\tilde{G}_{b+1}^{i, j}-\tilde{G}_{b}^{i, j}\right\|^{2}>\Delta^{3 / 2} \hat{C}_{\gamma}\right\} \subseteq\left\{N^{-1}\left|\mathcal{I}_{N}\right| \notin\left[c_{1} \Delta / 2, c_{1} \Delta(M+1)\right]\right\} \\
& \cup\left\{N^{-1}\left|\mathcal{I}_{N}\right| \in\left[c_{1} \Delta / 2, c_{1} \Delta(M+1)\right] \text { and }\left\|\tilde{\mathbf{J}}_{N}\right\|>3 \sqrt{c_{1} \Delta / 2}\right\} .
\end{aligned}
$$

It follows from basic properties of Poisson Processes (noted in Lemma 8.1) that the probability of the first term on the right hand side is exponentially decaying. It thus remains to prove that

$$
\varlimsup_{N \rightarrow \infty} N^{-1} \log \mathbb{P}\left(\left|\mathcal{I}_{N}\right| \in\left[c_{1} \Delta / 2, c_{1} \Delta(M+1)\right] \text { and }\left\|\tilde{\mathbf{J}}_{N}\right\|>3 \sqrt{c_{1} \Delta / 2}\right)<0
$$

Define $\overline{\mathbf{J}}_{N}$ to be the $\left|\mathcal{I}_{N}\right| \times\left|\mathcal{I}_{N}\right|$ square matrix with elements $\left|\mathcal{I}_{N}\right|^{-\frac{1}{2}} J^{j k}$ : that is, $\overline{\mathbf{J}}_{N}=\sqrt{N}\left|\mathcal{I}_{N}\right|^{-\frac{1}{2}} \tilde{\mathbf{J}}_{N}$. This means that

$$
\left\{\left\|\tilde{\mathbf{J}}_{N}\right\| \geq 3 \sqrt{c_{1} \Delta / 2} \text { and } N^{-1}\left|\mathcal{I}_{N}\right| \in\left[c_{1} \Delta / 2, c_{1} \Delta(M+1)\right]\right\} \subseteq\left\{\left\|\overline{\mathbf{J}}_{N}\right\| \geq 3 \text { and } N^{-1}\left|\mathcal{I}_{N}\right| \in\left[c_{1} \Delta / 2, c_{1} \Delta(M+1)\right]\right\} .
$$

Notice that the (random) indices in $\mathcal{I}_{N}$ are independent of the static connections $\left\{J^{j k}\right\}_{j, k \in \mathbb{Z}^{+}}$since the Poisson Processes $\left\{Y^{i, j}(t)\right\}$ are Markovian and independent of the static connections. We can now use known bounds on the dominant eigenvalue of random matrices (as noted in (3) of Lemma 3.2) to obtain that

$$
\begin{aligned}
\varlimsup_{N \rightarrow \infty} N^{-1} \log \mathbb{P}( & \left.\left\|\overline{\mathbf{J}}_{N}\right\| \geq 3 \text { and } N^{-1}\left|\mathcal{I}_{N}\right| \in\left[c_{1} \Delta / 2, c_{1} \Delta(M+1)\right]\right) \\
& =\varlimsup_{N \rightarrow \infty} N^{-1} \log \mathbb{E}\left[\mathbb{P}\left(\left\|\overline{\mathbf{J}}_{N}\right\| \geq 3 \text { and } N^{-1}\left|\mathcal{I}_{N}\right| \in\left[c_{1} \Delta / 2, c_{1} \Delta(M+1)\right] \mid \mathbf{Y}(t)\right)\right] \\
& \leq \varlimsup_{N \rightarrow \infty} N^{-1} \log \mathbb{E}\left[\chi\left\{\left|\mathcal{I}_{N}\right| \geq N c_{1} \Delta / 2 \mid\right\} \exp \left(-\left|\mathcal{I}_{N}\right| \Lambda_{J}\right)\right] \\
& <0
\end{aligned}
$$

as required, where the constant $\Lambda_{J}$ is defined in Lemma 3.2. 
We start with the bound of $\beta^{4}$ (which is defined in (158)): this can be thought of as the average 'crossvariation' between the spins and fields over the small time interval $\Delta$.

Lemma 6.2. For any $\bar{\epsilon}>0$, for sufficiently large $n$,

$$
\varlimsup_{N \rightarrow \infty} \sup _{1 \leq \mathfrak{q} \leq C_{\mathbf{n}}^{N}} N^{-1} \log \mathbb{P}\left(\tilde{\mu}^{N} \in \mathcal{V}_{\mathfrak{q}}^{N},\left|\beta^{4}\left(\boldsymbol{\alpha}, \tilde{\boldsymbol{\sigma}}_{b}, \tilde{\mathbf{G}}\right)\right| \geq \bar{\epsilon} \Delta\right)<0 .
$$

Proof. Now for some $z>0$,

$$
\begin{aligned}
\varlimsup_{N \rightarrow \infty} N^{-1} \log \mathbb{P}\left(\tilde{\mu}^{N} \in \mathcal{V}_{\mathfrak{q}}^{N},\left|\beta^{4}(\boldsymbol{\alpha}, \tilde{\boldsymbol{\sigma}}, \tilde{\mathbf{G}})\right| \geq \bar{\epsilon} \Delta\right) \leq \max \left\{\varlimsup_{N \rightarrow \infty} N^{-1} \log \mathbb{P}\left(\left|\mathcal{I}_{N}\right|>N\left(M c_{1} \Delta+z\right)\right),\right. \\
\left.\varlimsup_{N \rightarrow \infty} \sup _{1 \leq \mathfrak{q} \leq C_{\mathbf{n}}^{N}} N^{-1} \log \mathbb{P}\left(\left|\beta^{4}(\boldsymbol{\alpha}, \tilde{\boldsymbol{\sigma}}, \tilde{\mathbf{G}})\right| \geq \bar{\epsilon} \Delta,\left|\mathcal{I}_{N}\right| \leq N\left(M c_{t} \Delta+z\right), \tilde{\mu}^{N} \in \mathcal{V}_{\mathfrak{q}}^{N}\right)\right\}
\end{aligned}
$$

By Lemma 8.1,

$$
\varlimsup_{N \rightarrow \infty} N^{-1} \log \mathbb{P}\left(\left|\mathcal{I}_{N}\right|>N\left(M c_{1} \Delta+z\right)\right)<0 .
$$

It remains to prove that the second term on the right-hand-side of (179) is negative. Thanks to the identity in $(174)$, if $Y_{b}^{i, j}\left(c_{1} \Delta+z\right)=0$ for all $i \in I_{M}$ then $\tilde{\boldsymbol{\sigma}}_{b+1}=\tilde{\boldsymbol{\sigma}}_{b}$ and $\chi\left\{\tilde{\boldsymbol{\sigma}}_{b+1}^{j}=\boldsymbol{\alpha}\right\}-\chi\left\{\tilde{\boldsymbol{\sigma}}_{b}^{j}=\boldsymbol{\alpha}\right\}=0$. We thus have that

$$
\begin{aligned}
\beta^{4}\left(\boldsymbol{\alpha}, \tilde{\boldsymbol{\sigma}}_{b}, \tilde{\mathbf{G}}\right) & =N^{-1} \sum_{j \in \mathcal{I}_{N}}\left\{\phi^{a}\left(\tilde{\mathbf{G}}_{b+1}^{j}\right)-\phi^{a}\left(\tilde{\mathbf{G}}_{b}^{j}\right)\right\}\left\{\chi\left(\tilde{\boldsymbol{\sigma}}_{b+1}^{j}=\boldsymbol{\alpha}\right)-\chi\left(\tilde{\boldsymbol{\sigma}}_{b}^{j}=\boldsymbol{\alpha}\right)\right\} \\
& =N^{-1} \sum_{j \in \mathcal{I}_{N}} \phi_{i \in I_{M}}^{a}\left(\overline{\mathbf{G}}^{j}\right)\left(\tilde{\mathbf{G}}_{b+1}^{i, j}-\tilde{\mathbf{G}}_{b}^{i, j}\right)\left\{\chi\left(\tilde{\boldsymbol{\sigma}}_{b+1}^{j}=\boldsymbol{\alpha}\right)-\chi\left(\tilde{\boldsymbol{\sigma}}_{b}^{j}=\boldsymbol{\alpha}\right)\right\},
\end{aligned}
$$

for $\overline{\mathbf{G}}^{j}=\lambda_{j} \tilde{\mathbf{G}}_{b}^{j}+\left(1-\lambda_{j}\right) \tilde{\mathbf{G}}_{b+1}^{j}$, for some $\lambda_{j} \in[0,1]$, by the Taylor Remainder Theorem. It follows from (180) that if $\left|\mathcal{I}_{N}\right|<N \bar{\epsilon} \Delta / 4$, then since $\left|\phi^{a}\right| \leq 1$, it must necessarily be the case that

$$
\left|\beta^{4}(\boldsymbol{\alpha}, \tilde{\boldsymbol{\sigma}}, \tilde{\mathbf{G}})\right|<\bar{\epsilon} \Delta,
$$

as required. It thus suffices for us to prove that

$$
\varlimsup_{N \rightarrow \infty} N^{-1} \log \mathbb{P}\left(\tilde{\mu}^{N} \in \mathcal{V}_{\mathfrak{q}}^{N},\left|\beta^{4}(\boldsymbol{\alpha}, \tilde{\boldsymbol{\sigma}}, \tilde{\mathbf{G}})\right| \geq \bar{\epsilon} \Delta \text { and }\left|\mathcal{I}_{N}\right| \in\left[N \bar{\epsilon} \Delta / 4, N\left(M c_{1} \Delta+z\right)\right]\right)<0 .
$$

Using a union-of-events bound,

$$
\begin{aligned}
& \varlimsup_{N \rightarrow \infty} N^{-1} \log \mathbb{P}\left(\left|\beta^{4}\left(\boldsymbol{\alpha}, \tilde{\boldsymbol{\sigma}}_{b}, \tilde{\mathbf{G}}\right)\right| \geq \bar{\epsilon} \Delta \text { and }\left|\mathcal{I}_{N}\right| \in\left[N \bar{\epsilon} \Delta / 4, N\left(M c_{1} \Delta+z\right)\right]\right) \\
& \quad \leq \max \left\{\varlimsup_{N \rightarrow \infty} N^{-1} \log \mathbb{P}\left(\left\|\tilde{\mathbf{J}}_{N}\right\| \geq 3\left|\mathcal{I}_{N}\right|^{\frac{1}{2}} N^{-1 / 2} \text { and }\left|\mathcal{I}_{N}\right| \geq N \bar{\epsilon} \Delta / 4\right),\right. \\
& \left.\varlimsup_{N \rightarrow \infty} N^{-1} \log \mathbb{P}\left(\left|\beta^{4}\left(\boldsymbol{\alpha}, \tilde{\boldsymbol{\sigma}}_{b}, \tilde{\mathbf{G}}\right)\right| \geq \bar{\epsilon} \Delta \text { and }\left|\mathcal{I}_{N}\right| \epsilon\left[N \bar{\epsilon} \Delta / 4, N\left(M c_{1} \Delta+z\right)\right] \text { and }\|\tilde{\mathbf{J}}\| \leq 3\left|\mathcal{I}_{N}\right|^{\frac{1}{2}} N^{-1 / 2}\right)\right\},
\end{aligned}
$$

where $\tilde{\mathbf{J}}_{N}$ is the $\left|\mathcal{I}_{N}\right| \times\left|\mathcal{I}_{N}\right|$ square matrix with entries given by $\left\{N^{-1 / 2} J^{j k}\right\}_{j, k \in \mathcal{I}_{N}}$. Just as we proved in (178), for small enough $\Delta$,

$$
\varlimsup_{N \rightarrow \infty} N^{-1} \log \mathbb{P}\left(\left\|\tilde{\mathbf{J}}_{N}\right\| \geq 3\left|\mathcal{I}_{N}\right|^{\frac{1}{2}} N^{-1 / 2} \text { and }\left|\mathcal{I}_{N}\right| \geq N \bar{\epsilon} \Delta / 4\right) \leq \varlimsup_{N \rightarrow \infty} N^{-1} \log \mathbb{P}\left(\left\|\tilde{\mathbf{J}}_{N}\right\| \geq 3 N \bar{\epsilon} \Delta / 4\right)<0 .
$$

It thus suffices for us to prove that for $\Delta$ sufficiently small,

$$
\varlimsup_{N \rightarrow \infty} N^{-1} \log \mathbb{P}\left(\left|\beta^{4}\left(\boldsymbol{\alpha}, \tilde{\boldsymbol{\sigma}}_{b}, \tilde{\mathbf{G}}\right)\right| \geq \bar{\epsilon} \Delta \text { and }\left|\mathcal{I}_{N}\right| \epsilon\left[N \bar{\epsilon} \Delta / 4, N\left(M c_{1} \Delta+z\right)\right] \text { and }\|\tilde{\mathbf{J}}\| \leq 3\left|\mathcal{I}_{N}\right|^{\frac{1}{2}} N^{-1 / 2}\right)<0 .
$$

To this end, we obtain from (181) that, since $\left|\phi_{i}^{a}\right| \leq 1$, by the Cauchy-Schwarz Inequality,

$$
\begin{aligned}
\left|\beta^{4}\left(\boldsymbol{\alpha}, \tilde{\boldsymbol{\sigma}}_{b}, \tilde{\mathbf{G}}\right)\right|^{2} & \leq N^{-2} \sum_{j \in \mathcal{I}_{N}}\left(\tilde{\mathbf{G}}_{b+1}^{i, j}-\tilde{\mathbf{G}}_{b}^{i, j}\right)^{2} \times \sum_{j \in \mathcal{I}_{N}}\left\{\chi\left(\tilde{\boldsymbol{\sigma}}_{b+1}^{j}=\boldsymbol{\alpha}\right)-\chi\left(\tilde{\boldsymbol{\sigma}}_{b}^{j}=\boldsymbol{\alpha}\right)\right\}^{2} \\
& \leq N^{-2} \sum_{j \in \mathcal{I}_{N}}\left\|\tilde{\mathbf{J}}_{N}\right\|\left(\tilde{\sigma}_{b+1}^{i, j}-\tilde{\sigma}_{b}^{i, j}\right)^{2} \times \sum_{j \in \mathcal{I}_{N}}\left\{\chi\left(\tilde{\boldsymbol{\sigma}}_{b+1}^{j}=\boldsymbol{\alpha}\right)-\chi\left(\tilde{\boldsymbol{\sigma}}_{b}^{j}=\boldsymbol{\alpha}\right)\right\}^{2} .
\end{aligned}
$$


Now if $\left|\mathcal{I}_{N}\right| \leq N\left(M c_{1} \Delta+z\right)$ and $\left\|\tilde{\mathbf{J}}_{N}\right\| \leq 3\left|\mathcal{I}_{N}\right|^{\frac{1}{2}} N^{-1 / 2} \leq 3\left(M c_{1} \Delta+z\right)^{\frac{1}{2}}$, it must be that, (since $\left.\left(\tilde{\sigma}_{b+1}^{i, j}-\tilde{\sigma}_{b}^{i, j}\right)^{2} \leq 4\right)$,

$$
\left|\beta^{4}\left(\boldsymbol{\alpha}, \tilde{\boldsymbol{\sigma}}_{b}, \tilde{\mathbf{G}}\right)\right|^{2} \leq 16 N^{-2}\left|\mathcal{I}_{N}\right|^{2} 3\left(M c_{1} \Delta+z\right)^{\frac{1}{2}}
$$

We choose $z=\Delta$, and find that (184) implies that

$$
\left|\beta^{4}\left(\boldsymbol{\alpha}, \tilde{\boldsymbol{\sigma}}_{b}, \tilde{\mathbf{G}}\right)\right|^{2} \leq \text { Const } \times(\Delta)^{\frac{5}{2}} .
$$

This means that for $\Delta$ sufficiently small,

$$
\mathbb{P}\left(\left|\beta^{4}\left(\boldsymbol{\alpha}, \tilde{\boldsymbol{\sigma}}_{b}, \tilde{\mathbf{G}}\right)\right| \geq \bar{\epsilon} \Delta \text { and }\left|\mathcal{I}_{N}\right| \epsilon\left[N \bar{\epsilon} \Delta, N\left(M c_{1} \Delta+z\right)\right] \text { and }\|\tilde{\mathbf{J}}\| \leq 3\left|\mathcal{I}_{N}\right|^{\frac{1}{2}}\right)=0,
$$

which implies (183), as required.

Lemma 6.3. For any $\bar{\epsilon}>0$, for large enough $n$

$$
\sup _{0 \leq b<n} \sup _{\boldsymbol{\alpha} \in \mathcal{E}^{M}} \varlimsup_{N \rightarrow \infty} N^{-1} \log \sup _{1 \leq \mathfrak{q} \leq C_{\mathbf{n}}^{N}} \mathbb{P}\left(\mathcal{J}_{N}, \tilde{\mu}^{N} \in \mathcal{V}_{\mathfrak{q}}^{N}, \tilde{\tau}_{N}>t_{b}^{(n)},\left|\beta^{5}(\boldsymbol{\alpha}, \tilde{\boldsymbol{\sigma}}, \tilde{\mathbf{G}})\right| \geq \bar{\epsilon} \Delta / 18\right)<0
$$

Proof. Recall the definition of $\beta^{5}$ :

$$
\begin{aligned}
& \beta^{5}(\boldsymbol{\alpha}, \tilde{\boldsymbol{\sigma}}, \tilde{\mathbf{G}})=N^{-1} \sum_{j \in I_{N}} \chi\left\{\tilde{\boldsymbol{\sigma}}_{b}^{j}=\boldsymbol{\alpha}\right\}\left(\phi^{a}\left(\tilde{\mathbf{G}}_{b+1}^{j}\right)-\phi^{a}\left(\tilde{\mathbf{G}}_{b}^{j}\right)-\sum_{i \in I_{M}} \phi_{i}^{a}\left(\tilde{\mathbf{G}}_{b}^{j}\right)\left\{\tilde{G}_{b+1}^{i, j}-\tilde{G}_{b}^{i, j}\right\}\right. \\
&\left.-\frac{1}{2} \sum_{i, p \in I_{M}} \phi_{i p}^{a}\left(\tilde{\mathbf{G}}_{b}^{j}\right)\left\{\tilde{G}_{b+1}^{i, j}-\tilde{G}_{b}^{i, j}\right\}\left\{\tilde{G}_{b+1}^{p, j}-\tilde{G}_{b}^{p, j}\right\}\right) .
\end{aligned}
$$

If $\sup _{i \in I_{M}}\left|\tilde{G}_{b+1}^{i, j}-\tilde{G}_{b}^{i, j}\right| \leq(\Delta)^{2 / 5}$, then it follows from Taylor's Theorem that

$$
\begin{aligned}
& \mid \chi\left\{\tilde{\boldsymbol{\sigma}}_{b}^{j}=\boldsymbol{\alpha}\right\}\left(\phi^{a}\left(\tilde{\mathbf{G}}_{b+1}^{j}\right)-\phi^{a}\left(\tilde{\mathbf{G}}_{b}^{j}\right)-\sum_{i \in I_{M}} \phi_{i}^{a}\left(\tilde{\mathbf{G}}_{b}^{j}\right)\left\{\tilde{G}_{b+1}^{i, j}-\tilde{G}_{b}^{i, j}\right\}\right. \\
& \left.-\frac{1}{2} \sum_{i, p \in I_{M}} \phi_{i p}^{a}\left(\tilde{\mathbf{G}}_{b}^{j}\right)\left\{\tilde{G}_{b+1}^{i, j}-\tilde{G}_{b}^{i, j}\right\}\left\{\tilde{G}_{b+1}^{p, j}-\tilde{G}_{b}^{p, j}\right\}\right)\left|\leq \frac{1}{6}\right| \sum_{i, p, q \in I_{M}} \phi_{i p q}^{a}\left(\hat{\mathbf{G}}^{j}\right) \mid \tilde{G}_{b+1}^{i, j}-\tilde{G}_{b}^{i, j}\left\|\tilde{G}_{b+1}^{p, j}-\tilde{G}_{b}^{p, j}\right\| \tilde{G}_{b+1}^{q, j}-\tilde{G}_{b}^{q, j} \| \\
& \leq M^{3}(\Delta)^{6 / 5} / 6 \leq \Delta \bar{\epsilon} / 36,
\end{aligned}
$$

once $n$ is sufficiently large (since $\Delta=T n^{-1}$ ). Write $\tilde{I}_{N}=\left\{j \in I_{N}: \sup _{i \in I_{M}}\left|\tilde{G}_{b+1}^{i, j}-\tilde{G}_{b}^{i, j}\right| \leq(\Delta)^{2 / 5}\right\}$. Since the magnitude of $\phi$ and its first three derivatives are all upperbounded by 1 , it must be that there is a constant $C$ such that

$$
\begin{array}{r}
\left|\chi\left\{\tilde{\boldsymbol{\sigma}}_{b}^{j}=\boldsymbol{\alpha}\right\}\left(\phi^{a}\left(\tilde{\mathbf{G}}_{b+1}^{j}\right)-\phi^{a}\left(\tilde{\mathbf{G}}_{b}^{j}\right)-\sum_{i \in I_{M}} \phi_{i}^{a}\left(\tilde{\mathbf{G}}_{b}^{j}\right)\left\{\tilde{G}_{b+1}^{i, j}-\tilde{G}_{b}^{i, j}\right\}-\frac{1}{2} \sum_{i, p \in I_{M}} \phi_{i p}^{a}\left(\tilde{\mathbf{G}}_{b}^{j}\right)\left\{\tilde{G}_{b+1}^{i, j}-\tilde{G}_{b}^{i, j}\right\}\left\{\tilde{G}_{b+1}^{p, j}-\tilde{G}_{b}^{p, j}\right\}\right)\right| \\
\leq C\left(1+\sum_{p \in I_{M}}\left|\tilde{G}_{b+1}^{p, j}-\tilde{G}_{b}^{p, j}\right|^{2}\right) . \quad(187)
\end{array}
$$

The previous two equations imply that

$$
\left|\beta^{5}\left(\boldsymbol{\alpha}, \tilde{\boldsymbol{\sigma}}_{b}, \tilde{\mathbf{G}}_{b}\right)\right| \leq \Delta \bar{\epsilon} / 36+C N^{-1} \sum_{j \in I_{N}}\left[\chi\left\{\sup _{i \in I_{M}}\left|\tilde{G}_{b+1}^{i, j}-\tilde{G}_{b}^{i, j}\right|>(\Delta)^{2 / 5}\right\}\left(1+\sum_{p \in I_{M}}\left|\tilde{G}_{b+1}^{p, j}-\tilde{G}_{b}^{p, j}\right|^{2}\right)\right] .
$$

It thus suffices to prove that

$$
\varlimsup_{N \rightarrow \infty} N^{-1} \log \sup _{1 \leq \mathfrak{q} \leq C_{\mathbf{n}}^{N}} \mathbb{P}\left(\mathcal{J}_{N}, C N^{-1} \sum_{j \in I_{N}}\left[\chi\left\{\sup _{i \in I_{M}}\left|\tilde{G}_{b+1}^{i, j}-\tilde{G}_{b}^{i, j}\right|>(\Delta)^{2 / 5}\right\}\left(1+\sum_{p \in I_{M}}\left|\tilde{G}_{b+1}^{p, j}-\tilde{G}_{b}^{p, j}\right|^{2}\right)\right]>\Delta \bar{\epsilon} / 36\right)<0 .
$$


To establish the above equation, it suffices in turn to prove that (writing $\breve{\epsilon}=\bar{\epsilon} / 108$ ),

$$
\begin{aligned}
& \varlimsup_{N \rightarrow \infty} N^{-1} \log \sup _{1 \leq \mathfrak{q} \leq C_{\mathfrak{n}}^{N}} \mathbb{P}\left(\mathcal{J}_{N}, C N^{-1} \sum_{j \in \mathcal{I}_{N}}\left[\chi\left\{\sup _{i \in I_{M}}\left|\tilde{G}_{b+1}^{i, j}-\tilde{G}_{b}^{i, j}\right|>(\Delta)^{2 / 5}\right\}\left(1+\sum_{p \in I_{M}}\left|\tilde{G}_{b+1}^{p, j}-\tilde{G}_{b}^{p, j}\right|^{2}\right)\right]>\Delta \breve{\epsilon}\right)<0 \\
& \varlimsup_{N \rightarrow \infty} N^{-1} \log \sup _{1 \leq \mathfrak{q} \leq C_{\mathbf{n}}^{N}} \mathbb{P}\left(\mathcal{J}_{N}, C N^{-1} \sum_{j \in \mathcal{I}_{N}^{c}}\left[\chi\left\{\sup _{i \in I_{M}}\left|\tilde{G}_{b+1}^{i, j}-\tilde{G}_{b}^{i, j}\right|>(\Delta)^{2 / 5}\right\} \sum_{p \in I_{M}}\left|\tilde{G}_{b+1}^{p, j}-\tilde{G}_{b}^{p, j}\right|^{2}\right]>\Delta \breve{\epsilon}\right)<0 \\
& \varlimsup_{N \rightarrow \infty} N^{-1} \log \sup _{1 \leq \mathfrak{q} \leq C_{\mathfrak{n}}^{N}} \mathbb{P}\left(\mathcal{J}_{N}, C N^{-1} \sum_{j \in \mathcal{I}_{N}^{c}}\left[\chi\left\{\sup _{i \in I_{M}}\left|\tilde{G}_{b+1}^{i, j}-\tilde{G}_{b}^{i, j}\right|>(\Delta)^{2 / 5}\right\}\right]>\Delta \breve{\epsilon}\right)<0
\end{aligned}
$$

and we recall that $\mathcal{I}_{N}^{c}=\left\{j \in I_{N}: j \notin \mathcal{I}_{N}\right\}$. Starting with (189), observe that

$$
\begin{aligned}
\left\{N ^ { - 1 } \sum _ { j \in \mathcal { I } _ { N } } \left[\chi\left\{\sup _{i \in I_{M}}\left|\tilde{G}_{b+1}^{i, j}-\tilde{G}_{b}^{i, j}\right|>(\Delta)^{2 / 5}\right\}(1+\right.\right. & \left.\left.\left.\sum_{p \in I_{M}}\left|\tilde{G}_{b+1}^{p, j}-\tilde{G}_{b}^{p, j}\right|^{2}\right)\right]>\Delta \breve{\epsilon} C^{-1}\right\} \\
& \left.\subseteq\left\{N^{-1} \sum_{j \in \mathcal{I}_{N}} \sum_{p \in I_{M}}\left|\tilde{G}_{b+1}^{p, j}-\tilde{G}_{b}^{p, j}\right|^{2}\left(\Delta^{-1 / 5}+1\right)\right)>\Delta \breve{\epsilon} / C\right\}
\end{aligned}
$$

The probability of the right hand side is exponentially decaying (for large enough $n$ ), as a consequence of Lemma 6.1. The inequalities (190) and (191) are established in Lemma 6.4.

Lemma 6.4. For any $\epsilon>0$, for sufficiently large $n \in \mathbb{Z}^{+}$(and recalling that $\Delta=T / n$ ),

$$
\begin{array}{r}
\varlimsup_{N \rightarrow \infty} \sup _{1 \leq \mathfrak{q} \leq C_{\mathfrak{n}}^{N}} N^{-1} \log \mathbb{P}\left(N^{-1} \sum_{j \in \mathcal{I}_{N}^{c}} \chi\left\{\left|\tilde{G}_{b+1}^{i, j}-\tilde{G}_{b}^{i, j}\right| \geq(\Delta)^{2 / 5} \text { for some } i \in I_{M}\right\} \geq \epsilon \Delta\right)<0 \\
\varlimsup_{N \rightarrow \infty} \sup _{1 \leq \mathfrak{q} \leq C_{\mathfrak{n}}^{N}} N^{-1} \log \mathbb{P}\left(N^{-1} \sum_{j \in \mathcal{I}_{N}^{c}} \chi\left\{\left|\tilde{G}_{b+1}^{i, j}-\tilde{G}_{b}^{i, j}\right| \geq(\Delta)^{2 / 5} \text { for some } i \epsilon I_{M}\right\}\left|\tilde{G}_{b+1}^{i, j}-\tilde{G}_{b}^{i, j}\right|^{2} \geq \epsilon \Delta\right)<0 .
\end{array}
$$

Proof. The proofs are very similar and so we only include the second result. It follows from Lemma 3.1 that

$$
\begin{aligned}
\varlimsup_{N \rightarrow \infty} N^{-1} \log \mathbb{P}\left(N^{-1} \sum_{j \in \mathcal{I}_{N}^{c}} \chi\left\{\left|\tilde{G}_{b+1}^{i, j}-\tilde{G}_{b}^{i, j}\right| \geq(\Delta)^{2 / 5} \text { for some } i \in I_{M}\right\}\left|\tilde{G}_{b+1}^{i, j}-\tilde{G}_{b}^{i, j}\right|^{2} \geq \epsilon \Delta\right) \\
\leq \max \left\{\varlimsup _ { N \rightarrow \infty } N ^ { - 1 } \operatorname { l o g } \mathbb { P } \left(N^{-1} \sum_{j \in \mathcal{I}_{N}^{c}, p \in I_{M}} \chi\left\{\left|\tilde{G}_{b+1}^{i, j}-\tilde{G}_{b}^{i, j}\right| \geq(\Delta)^{2 / 5} \text { for some } i \epsilon I_{M}\right\}\left|\tilde{G}_{b+1}^{p, j}-\tilde{G}_{b}^{p, j}\right|^{2} \geq \epsilon \Delta\right.\right. \\
\left.\left.\quad \text { and } N^{-1}\left|\mathcal{I}_{N}\right| \in\left[c_{1} \Delta / 2, c_{1} \Delta(M+1)\right]\right), \varlimsup_{N \rightarrow \infty} N^{-1} \log \mathbb{P}\left(N^{-1}\left|\mathcal{I}_{N}\right| \notin\left[c_{1} \Delta / 2, c_{1} \Delta(M+1)\right]\right)\right\} .
\end{aligned}
$$

By Lemma 8.1, $\varlimsup_{N \rightarrow \infty} N^{-1} \log \mathbb{P}\left(N^{-1}\left|\mathcal{I}_{N}\right| \notin\left[c_{1} \Delta / 2, c_{1} \Delta(M+1)\right]\right)<0$ as required.

We write $\tilde{H}^{i, j}=\tilde{G}_{b+1}^{i, j}-\tilde{G}_{b}^{i, j}$. By Chernoff's Inequality,

$$
\begin{aligned}
& \mathbb{P}\left(N^{-1} \sum_{j \in \mathcal{I}_{N}^{c}, p \in I_{M}} \chi\left\{\left|\tilde{H}^{i, j}\right| \geq(\Delta)^{2 / 5} \text { for some } i \in I_{M}\right\}\left|\tilde{H}^{p, j}\right|^{2} \geq \epsilon \Delta\right. \\
& \left.\quad \text { and } N^{-1}\left|\mathcal{I}_{N}\right| \epsilon\left[c_{1} \Delta / 2, c_{1} \Delta(M+1)\right]\right) \leq \exp (-N \epsilon \Delta) \times \\
& \mathbb{E}\left[\chi\left\{N^{-1}\left|\mathcal{I}_{N}\right| \in\left[c_{1} \Delta / 2, c_{1} \Delta(M+1)\right]\right\} \exp \left(\sum_{j \in \mathcal{I}_{N}^{c}, p \in I_{M}} \chi\left\{\left|\tilde{H}^{i, j}\right| \geq(\Delta)^{2 / 5} \text { for some } i \in I_{M}\right\}\left|\tilde{H}^{p, j}\right|^{2}\right)\right] .
\end{aligned}
$$

In the above expectation, $\tilde{\boldsymbol{\sigma}}$ (which determines the indices $\mathcal{I}_{N}$ ) is independent of $\mathbf{J}$. Furthermore, conditionally on $\tilde{\boldsymbol{\sigma}}, \tilde{G}^{i, j}$ is independent of $\tilde{G}^{p, k}$ if $j \neq k$ and $j, k \in \mathcal{I}_{N}^{c}$. This last fact is immediate from the definition of the indices in $\mathcal{I}_{N}^{c}$ : the coefficients of common edges are zero. We thus see that the conditional moments 
are

$$
\begin{aligned}
\mathbb{E}\left[\tilde{H}^{i, j} \mid \tilde{\boldsymbol{\sigma}}\right] & =0 \\
\mathbb{E}\left[\left(\tilde{H}^{i, j}\right)^{2}\left|N^{-1}\right| \mathcal{I}_{N} \mid \in\left[c_{1} \Delta / 2, c_{1} \Delta(M+1)\right]\right] & \leq N^{-1} \sum_{j \in I_{N}}\left(\tilde{\sigma}_{b+1}^{i, j}-\tilde{\sigma}_{b}^{i, j}\right)^{2}+N^{-1} \\
& \leq 4 N^{-1}\left|\mathcal{I}_{N}\right|+N^{-1} \\
& \leq 4 c_{1} \Delta(M+1)+N^{-1},
\end{aligned}
$$

as long as $N^{1}\left|\mathcal{I}_{N}\right| \leq c_{1} \Delta(M+1)$. Standard Gaussian properties therefore dictate that as long as $N^{-1}\left|\mathcal{I}_{N}\right| \leq$ $c_{1} \Delta(M+1)$, there exists a constant $k>0$ such that for all sufficiently small $\Delta$,

$\mathbb{E}^{\gamma}\left[\exp \left(\sum_{p \in I_{M}} \chi\left\{\left|\tilde{H}^{i, j}\right| \geq(\Delta)^{2 / 5}\right.\right.\right.$ for some $\left.\left.\left.i \in I_{M}\right\}\left|\tilde{H}^{p, j}\right|^{2}\right) \mid \tilde{\boldsymbol{\sigma}}\right] \leq 1+\exp \left(-k(\Delta)^{-1 / 5}\right) \leq \exp \left(\exp \left\{-k(\Delta)^{-1 / 5}\right\}\right)$

This means that

$$
\begin{array}{r}
\exp (-N \epsilon \Delta) \mathbb{E}\left[\chi\left\{N^{-1}\left|\mathcal{I}_{N}\right| \leq c_{1} \Delta(M+1)\right\} \exp \left(\sum_{j \in \mathcal{I}_{N}^{c}, p \in I_{M}} \chi\left\{\left|\tilde{H}^{i, j}\right| \geq(\Delta)^{2 / 5} \text { for some } i \in I_{M}\right\}\left|\tilde{H}^{p, j}\right|^{2}\right)\right] \\
\leq \exp \left(-N \epsilon \Delta+N \exp \left\{-k(\Delta)^{-1 / 5}\right\}\right)
\end{array}
$$

For small enough $\Delta$, the right hand side is exponentially decaying, as required.

\subsection{Bounds using Concentration Inequalities for Poisson Processes}

Lemma 6.5. For any $\tilde{\epsilon}>0$, for all large enough $n$ (and therefore small $\Delta=T / n$ ), we can find $\mathfrak{n}_{0}(n) \in \mathbb{Z}^{+}$ such that for all $\mathfrak{n} \geq \mathfrak{n}_{0}(n)$,

$$
\sup _{0 \leq b<n} \varlimsup_{N \rightarrow \infty} \sup _{1 \leq \mathfrak{q} \leq C_{\mathfrak{n}}^{N}} N^{-1} \log \sup _{\boldsymbol{\alpha} \in \mathcal{E}^{M}} \mathbb{P}\left(\mathcal{J}_{N}, \tilde{\mu}^{N}(\tilde{\boldsymbol{\sigma}}, \tilde{\mathbf{G}}) \in \mathcal{V}_{\mathfrak{q}}^{N},\left|\beta^{1}\left(\boldsymbol{\alpha}, \tilde{\boldsymbol{\sigma}}_{b}, \mathbf{G}_{b}\right)\right| \geq \tilde{\epsilon} \Delta\right)<0
$$

Proof. We prove that

$$
\sup _{0 \leq b<n} \varlimsup_{N \rightarrow \infty} \sup _{1 \leq \mathfrak{q} \leq C_{\mathfrak{n}}^{N}} N^{-1} \log \sup _{\boldsymbol{\alpha} \in \mathcal{E}^{M}} \mathbb{P}\left(\mathcal{J}_{N}, \tilde{\mu}^{N}(\tilde{\boldsymbol{\sigma}}, \tilde{\mathbf{G}}) \in \mathcal{V}_{\mathfrak{q}}^{N}, \beta^{1}\left(\boldsymbol{\alpha}, \tilde{\boldsymbol{\sigma}}_{b}, \mathbf{G}_{b}\right) \geq \tilde{\epsilon} \Delta\right)<0
$$

The proof of the reverse inequality, i.e.

$$
\sup _{0 \leq b<n} \varlimsup_{N \rightarrow \infty} \sup _{1 \leq \mathfrak{q} \leq C_{\mathfrak{n}}^{N}} N^{-1} \log \sup _{\boldsymbol{\alpha} \in \mathcal{E}^{M}} \mathbb{P}\left(\mathcal{J}_{N}, \tilde{\mu}^{N}(\tilde{\boldsymbol{\sigma}}, \tilde{\mathbf{G}}) \in \mathcal{V}_{\mathfrak{q}}^{N}, \beta^{1}\left(\boldsymbol{\alpha}, \tilde{\boldsymbol{\sigma}}_{b}, \mathbf{G}_{b}\right) \leq-\tilde{\epsilon} \Delta\right)<0
$$

is analogous. One can decompose

$$
\beta^{1}(\boldsymbol{\alpha}, \tilde{\boldsymbol{\sigma}}, \tilde{\mathbf{G}})=\mathcal{Z}_{\mathfrak{q}}^{N}+\Delta \mathcal{U}_{\mathfrak{q}}^{N}
$$

where

$$
\begin{aligned}
& \mathcal{Z}_{\mathfrak{q}}^{N}=N^{-1} \sum_{j \in I_{N}} \phi^{a}\left(\tilde{\mathbf{G}}_{b}^{j}\right)\left[\chi\left\{\tilde{\boldsymbol{\sigma}}_{b+1}^{j}=\boldsymbol{\alpha}\right\}-\chi\left\{\tilde{\boldsymbol{\sigma}}_{b}^{j}=\boldsymbol{\alpha}\right\}\right. \\
&\left.-\Delta \sum_{i \in I_{M}, j \in I_{N}}\left\{c\left(-\tilde{\sigma}^{i, j}, \tilde{\mathfrak{G}}_{\mathfrak{q}, t_{b}^{i, j)}}^{i, j}\right) \chi\left\{\tilde{\boldsymbol{\sigma}}_{b}^{j}=\boldsymbol{\alpha}[i]\right\}-c\left(\tilde{\sigma}^{i, j}, \tilde{\mathfrak{G}}_{\mathfrak{q}, t_{b}^{i, j)}}^{i, j}\right) \chi\left\{\tilde{\boldsymbol{\sigma}}_{b}^{j}=\boldsymbol{\alpha}\right\}\right\}\right] \text { and } \\
& \mathcal{U}_{\mathfrak{q}}^{N}=\mathbb{E}^{\hat{\mu}_{b}^{N}\left(\tilde{\boldsymbol{\sigma}}, \tilde{\mathfrak{G}}_{\mathfrak{q}}\right)}[H]-\mathbb{E}^{\hat{\mu}_{b}^{N}(\tilde{\boldsymbol{\sigma}}, \tilde{\mathbf{G}})}[H] \text { where } H: \mathcal{E}^{M} \times \mathbb{R}^{M} \rightarrow \mathbb{R} \text { is of the form } \\
& H(\boldsymbol{\zeta}, \mathbf{x})=\sum_{i \in I_{M}}\left\{c\left(-\zeta^{i}, x^{i}\right) \chi\{\boldsymbol{\zeta}=\boldsymbol{\alpha}[i]\}-c\left(\zeta^{i}, x^{i}\right) \chi\{\boldsymbol{\zeta}=\boldsymbol{\alpha}\}\right\} .
\end{aligned}
$$


Thanks to Lemma 4.1, for large enough $\mathfrak{n}$, if $\tilde{\mu}^{N}(\tilde{\boldsymbol{\sigma}}, \tilde{\mathbf{G}}) \in \mathcal{V}_{\mathfrak{q}}^{N}$ then necessarily

$$
\left|\mathcal{U}_{\mathfrak{q}}^{N}\right| \leq \tilde{\epsilon} \Delta / 2 \text {. }
$$

Suppose that $\sum_{i \in I_{M}} Y_{b}^{i, j}\left(c_{1} \Delta\right) \leq 1$ (recall the definition in (173)). In this case, at most one of the spins $\left\{\tilde{\sigma}_{s}^{i, j}\right\}_{i \in I_{M}}$ flips once over the time interval $\left[t_{b}^{(n)}, t_{b+1}^{(n)}\right]$. In this case,

$$
\begin{array}{r}
\chi\left\{\tilde{\boldsymbol{\sigma}}_{b+1}^{j}=\boldsymbol{\alpha}\right\}-\chi\left\{\tilde{\boldsymbol{\sigma}}_{b}^{j}=\boldsymbol{\alpha}\right\}-\Delta \sum_{i \in I_{M}, j \in I_{N}}\left\{c\left(-\tilde{\sigma}_{b}^{i, j}, \tilde{\mathfrak{G}}_{\mathfrak{q}, t_{b}^{(n)}}^{i, j}\right) \chi\left\{\tilde{\boldsymbol{\sigma}}_{b}^{j}=\boldsymbol{\alpha}[i]\right\}-c\left(\tilde{\sigma}_{b}^{i, j}, \tilde{\mathfrak{G}}_{\mathfrak{q}, t_{b}^{i, j}}^{(n)}\right) \chi\left\{\tilde{\boldsymbol{\sigma}}_{b}^{j}=\boldsymbol{\alpha}\right\}\right\} \\
=\sum_{i \in I_{M}}\left\{Y_{b}^{i, j}\left(\int_{t_{b}^{(n)}}^{t_{b+1}^{(n)}} c\left(\tilde{\sigma}_{s}^{i, j}, \tilde{\mathfrak{G}}_{s}^{i, j}\right) d s\right) \chi\left\{\tilde{\boldsymbol{\sigma}}_{b}^{j}=\boldsymbol{\alpha}[i]\right\}-Y_{b}^{i, j}\left(\int_{t_{b}^{(n)}}^{t_{b+1}^{(n)}} c\left(\tilde{\sigma}_{s}^{i, j}, \tilde{\mathfrak{G}}_{s}^{i, j}\right) d s\right) \chi\left\{\tilde{\boldsymbol{\sigma}}_{b}^{j}=\boldsymbol{\alpha}\right\}\right. \\
\left.-\Delta\left\{c\left(-\tilde{\sigma}_{b}^{i, j}, \tilde{\mathfrak{G}}_{\mathfrak{q}, t_{b}^{i, j}}^{i, j}\right) \chi\left\{\tilde{\boldsymbol{\sigma}}_{b}^{j}=\boldsymbol{\alpha}[i]\right\}-c\left(\tilde{\sigma}^{i, j}, \tilde{\mathfrak{G}}_{\mathfrak{q}, t_{b}^{(n)}}^{i, j}\right) \chi\left\{\tilde{\boldsymbol{\sigma}}_{b}^{j}=\boldsymbol{\alpha}\right\}\right\}\right\} .
\end{array}
$$

Conversely if $\sum_{i \in I_{M}} Y_{b}^{i, j}\left(c_{1} \Delta\right) \geq 2$, then

$$
\begin{aligned}
-\sum_{i \in I_{M}}\left\{Y _ { b } ^ { i , j } ( \int _ { t _ { b } ^ { ( n ) } } ^ { t _ { b + 1 } ^ { ( n ) } } c ( \tilde { \sigma } _ { s } ^ { i , j } , \tilde { \mathfrak { G } } _ { s } ^ { i , j } ) d s ) \chi \left\{\tilde{\boldsymbol{\sigma}}_{b}^{j}\right.\right. & \left.=\boldsymbol{\alpha}[i]\}-Y_{b}^{i, j}\left(\int_{t_{b}^{(n)}}^{t_{b+1}^{(n)}} c\left(\tilde{\sigma}_{s}^{i, j}, \tilde{\mathfrak{G}}_{s}^{i, j}\right) d s\right) \chi\left\{\tilde{\boldsymbol{\sigma}}_{b}^{j}=\boldsymbol{\alpha}\right\}\right\} \\
& +\chi\left\{\tilde{\boldsymbol{\sigma}}_{b+1}^{j}=\boldsymbol{\alpha}\right\}-\chi\left\{\tilde{\boldsymbol{\sigma}}_{b}^{j}=\boldsymbol{\alpha}\right\} \leq 4 \sum_{i \in I_{M}} Y_{b}^{i, j}\left(c_{1} \Delta\right) \chi\left\{Y_{b}^{i, j}\left(c_{1} \Delta\right) \geq 2\right\} .
\end{aligned}
$$

We thus find that

$$
\begin{gathered}
\left\{\mathcal{Z}_{\mathfrak{q}}^{N}>\frac{\tilde{\epsilon} \Delta}{2}\right\} \subseteq\left\{N^{-1} \sum_{j \in I_{N}, i \in I_{M}} \chi\left\{\tilde{\boldsymbol{\sigma}}_{b}^{j}=\boldsymbol{\alpha}\right\}\left(\int_{t_{b}^{(n)}}^{t_{b+1}^{(n)}} c\left(\tilde{\sigma}_{s}^{i, j}, \tilde{\mathfrak{G}}_{s}^{i, j}\right) d s-Y_{b}^{i, j}\left(\int_{t_{b}^{(n)}}^{t_{b+1}^{(n)}} c\left(\tilde{\sigma}_{s}^{i, j}, \tilde{\mathfrak{G}}_{s}^{i, j}\right) d s\right)\right) \geq \frac{\tilde{\epsilon} \Delta}{8}\right\} \\
\cup\left\{N^{-1} \sum_{j \in I_{N}, i \in I_{M}} \chi\left\{\tilde{\boldsymbol{\sigma}}_{b}^{j}=\boldsymbol{\alpha}[i]\right\}\left(Y_{b}^{i, j}\left(\int_{t_{b}^{(n)}}^{t_{b+1}^{(n)}} c\left(\tilde{\sigma}_{s}^{i, j}, \tilde{\mathfrak{G}}_{s}^{i, j}\right) d s\right)-\int_{t_{b}^{(n)}}^{t_{b+1}^{(n)}} c\left(\tilde{\sigma}_{s}^{i, j}, \tilde{\mathfrak{G}}_{s}^{i, j}\right) d s\right) \geq \frac{\tilde{\epsilon} \Delta}{8}\right\} \\
\cup\left\{N^{-1} \sum_{j \in I_{N}, i \in I_{M}} \chi\left\{\tilde{\boldsymbol{\sigma}}_{b}^{j}=\boldsymbol{\alpha}\right\} \int_{t_{b}^{(n)}}^{t_{b+1}^{(n)}}\left\{c\left(\tilde{\sigma}_{b}^{i, j}, \tilde{\mathfrak{G}}_{b}^{i, j}\right)-c\left(\tilde{\sigma}_{s}^{i, j}, \tilde{\mathfrak{G}}_{s}^{i, j}\right)\right\} d s\right. \\
\left.-N^{-1} \sum_{j \in I_{N}, i \in I_{M}} \chi\left\{\tilde{\boldsymbol{\sigma}}_{b}^{j}=\boldsymbol{\alpha}[i]\right\} \int_{t_{b}^{(n)}}^{t_{b+1}^{(n)}}\left\{c\left(\tilde{\sigma}_{b}^{i, j}, \tilde{\mathfrak{G}}_{b}^{i, j}\right)-c\left(\tilde{\sigma}_{s}^{i, j}, \tilde{\mathfrak{G}}_{s}^{i, j}\right)\right\} d s \geq \frac{\tilde{\epsilon} \Delta}{8}\right\} \\
\cup\left\{4 N^{-1} \sum_{j \in I_{N}, i \in I_{M}} Y_{b}^{i, j}\left(c_{1} \Delta\right) \chi\left\{Y_{b}^{i, j}\left(c_{1} \Delta\right) \geq 2\right\} \geq \frac{\tilde{\epsilon} \Delta}{8}\right\} .
\end{gathered}
$$

The probability of each of the first two terms on the right hand side is exponentially decaying thanks to (i) of Lemma 8.2. For the third term, one easily shows that as long as the event $\mathcal{J}_{N}$ holds,

$$
\begin{aligned}
& \quad N^{-1} \sum_{j \in I_{N}, i \in I_{M}} \chi\left\{\tilde{\boldsymbol{\sigma}}_{b}^{j}=\boldsymbol{\alpha}\right\} \int_{t_{b}^{(n)}}^{t_{b+1}^{(n)}}\left\{c\left(\tilde{\sigma}_{b}^{i, j}, \tilde{\mathfrak{G}}_{b}^{i, j}\right)-c\left(\tilde{\sigma}_{s}^{i, j}, \tilde{\mathfrak{G}}_{s}^{i, j}\right)\right\} d s \\
& -N^{-1} \sum_{j \in I_{N}, i \in I_{M}} \chi\left\{\tilde{\boldsymbol{\sigma}}_{b}^{j}=\boldsymbol{\alpha}[i]\right\} \int_{t_{b}^{(n)}}^{t_{b+1}^{(n)}}\left\{c\left(\tilde{\sigma}_{b}^{i, j}, \tilde{\mathfrak{G}}_{b}^{i, j}\right)-c\left(\tilde{\sigma}_{s}^{i, j}, \tilde{\mathfrak{G}}_{s}^{i, j}\right)\right\} d s \mid \leq \operatorname{Const}\left(N^{-1} \sum_{j \in I_{N}, i \in I_{M}} \chi\left\{Y_{b}^{i, j}\left(c_{1} \Delta t\right) \geq 1\right\}\right)^{2} .
\end{aligned}
$$

Thanks to (ii) of Lemma 8.1, one finds that the probability of the RHS of the above equation exceeding $\tilde{\epsilon} \Delta / 8$ is exponentially decaying in $N$, once $\Delta$ is small enough. For the last term on the RHS of (202), by Chernoff's Inequality, for a constant $u>0$,

$$
\begin{aligned}
\mathbb{P}\left(4 N ^ { - 1 } \sum _ { j \in I _ { N } , i \in I _ { M } } Y _ { b } ^ { i , j } ( c _ { 1 } \Delta ) \chi \left\{Y_{b}^{i, j}\left(c_{1} \Delta\right)\right.\right. & \geq 2\} \geq \tilde{\epsilon} \Delta / 8) \\
\leq & \mathbb{E}\left[\exp \left(4 u N^{-1} \sum_{j \in I_{N}, i \in I_{M}} Y_{b}^{i, j}\left(c_{1} \Delta\right) \chi\left\{Y_{b}^{i, j}\left(c_{1} \Delta\right) \geq 2\right\}-u \tilde{\epsilon} \Delta / 8\right)\right]
\end{aligned}
$$


Now for any positive integer $k$, thanks to the renewal property of Poisson Processes,

$$
\mathbb{P}\left(Y_{b}^{i, j}\left(c_{1} \Delta\right)=k\right) \leq \mathbb{P}\left(Y_{b}^{i, j}\left(c_{1} \Delta\right)=1\right)^{k}=\left\{c_{1} \Delta \exp \left(-c_{1} \Delta\right)\right\}^{k},
$$

since $Y_{b}^{i, j}\left(c_{1} \Delta\right)$ is Poisson-distributed. We take $n$ sufficiently large that $\Delta \leq\left(c_{1}\right)^{-1}$, and $4 u=c_{1} \Delta / 2$, and we obtain that

$$
\begin{aligned}
\mathbb{E}\left[\exp \left(4 u N^{-1} Y_{b}^{i, j}\left(c_{1} \Delta\right) \chi\left\{Y_{b}^{i, j}\left(c_{1} \Delta\right) \geq 2\right\}-u \tilde{\epsilon} \Delta / 8\right)\right] & \leq \exp (-u \tilde{\epsilon} \Delta / 8) \sum_{k=2}^{\infty} \exp \left(-k c_{1} \Delta / 2\right) \\
& =\exp \left(-u \tilde{\epsilon} \Delta / 8-c_{1} \Delta\right)\left(1-\exp \left(-c_{1} \Delta / 2\right)\right)^{-1}
\end{aligned}
$$

by summing the geometric series. Since the processes $Y_{b}^{i, j}$ are independent, we find that the RHS of (203) is exponentially decaying in $N$, as required.

Lemma 6.6. For any $\bar{\epsilon}$, for all sufficiently large $n$,

$$
\begin{aligned}
& \varlimsup_{N \rightarrow \infty} \sup _{1 \leq \mathfrak{q} \leq C_{\mathfrak{n}}^{N}} N^{-1} \log \mathbb{P}\left(\mathcal{J}_{N}, \tilde{\mu}^{N} \in \mathcal{V}_{\mathfrak{q}}^{N}, \tilde{\tau}_{N}>t_{b}^{(n)},\left|\beta^{11}(\boldsymbol{\alpha}, \tilde{\boldsymbol{\sigma}}, \tilde{\mathbf{G}})\right| \geq \bar{\epsilon} \Delta\right)<0 \\
& \varlimsup_{N \rightarrow \infty} \sup _{1 \leq \mathfrak{q} \leq C_{\mathfrak{n}}^{N}} N^{-1} \log \mathbb{P}\left(\mathcal{J}_{N}, \tilde{\mu}^{N} \in \mathcal{V}_{\mathfrak{q}}^{N}, \tilde{\tau}_{N}>t_{b}^{(n)},\left|\beta^{7}(\boldsymbol{\alpha}, \tilde{\boldsymbol{\sigma}}, \tilde{\mathbf{G}})\right| \geq \bar{\epsilon} \Delta\right)<0
\end{aligned}
$$

Proof. Now since $\left|\phi_{i i}^{a}\right| \leq 1,\left\{\left|\beta^{11}(\boldsymbol{\alpha}, \tilde{\boldsymbol{\sigma}}, \tilde{\mathbf{G}})\right| \geq \bar{\epsilon} \Delta\right\} \subseteq\left\{\left|2 \Delta L_{i i}^{\hat{\mu}_{b}^{N}}-\tilde{L}_{b}^{i i}\right| \geq \bar{\epsilon} \Delta\right\}$. The probability of this event is exponentially decaying, thanks to Lemma 6.7 .

The proof of (205) is similar: one compares the definition of $\tilde{\mathbf{m}}_{b}$ in (162) to the definition of $\mathbf{m}^{\hat{\mu}_{b}^{N}}$ in (30). Note that the condition $\tilde{\tau}_{N}>t_{b}^{(n)}$ implies that $\tilde{\mathbf{H}}_{b}=\mathbf{H}^{\hat{\mu}_{b}^{N}}$ (see the definition in (29)) and also $\tilde{\boldsymbol{v}}_{b}=\boldsymbol{v}^{\hat{\mu}_{b}^{N}}$. One therefore finds that

$$
\begin{aligned}
\left|\beta^{7}(\boldsymbol{\alpha}, \tilde{\boldsymbol{\sigma}}, \tilde{\mathbf{G}})\right| & \leq N^{-1} \sum_{j \in I_{N}} \sum_{i \in I_{M}}\left|\tilde{m}_{b}^{i, j}-\Delta m^{\hat{\mu}_{b}^{N}, i}\left(\tilde{\boldsymbol{\sigma}}_{b}^{j}, \tilde{\mathbf{G}}_{b}^{j}\right)\right| \\
\tilde{\mathbf{m}}_{b}^{j}-\Delta \mathbf{m}^{\hat{\mu}_{b}^{N}}\left(\tilde{\boldsymbol{\sigma}}_{b}^{j}, \tilde{\mathbf{G}}_{b}^{j}\right) & =-\left(\tilde{\mathbf{L}}_{b}-2 \Delta \mathbf{L}^{\hat{\mu}_{b}^{N}}\right) \tilde{\mathbf{H}}_{b} \tilde{\mathbf{G}}_{b}^{j}-\mathfrak{s}\left(\tilde{\boldsymbol{\kappa}}_{b}-2 \Delta \boldsymbol{\kappa}^{\hat{\mu}_{b}^{N}}\right) \tilde{\mathbf{H}}_{b} \tilde{\boldsymbol{\sigma}}_{b}^{j}+\mathfrak{s}\left(\tilde{\mathbf{L}}_{b}-2 \Delta \mathbf{L}^{\hat{\mu}_{b}^{N}}\right) \tilde{\mathbf{H}}_{b} \tilde{\boldsymbol{v}}_{b} \tilde{\mathbf{H}}_{b} \tilde{\boldsymbol{\sigma}}_{b}^{j} .
\end{aligned}
$$

Thus

$$
\left\|\tilde{\mathbf{m}}_{b}^{j}-\Delta \mathbf{m}^{\hat{\mu}_{b}^{N}}\left(\tilde{\boldsymbol{\sigma}}_{b}^{j}, \tilde{\mathbf{G}}_{b}^{j}\right)\right\| \leq\left\|\tilde{\mathbf{L}}_{b}-2 \Delta \mathbf{L}^{\hat{\mu}_{b}^{N}}\right\|\left\|\tilde{\mathbf{H}}_{b}\right\|\left\|\tilde{\mathbf{G}}_{b}^{j}\right\|+\left\|\tilde{\boldsymbol{\kappa}}_{b}-2 \Delta \boldsymbol{\kappa}^{\hat{\mu}_{b}^{N}}\right\|\left\|\tilde{\mathbf{H}}_{b}\right\|\left\|\tilde{\boldsymbol{\sigma}}_{b}^{j}\right\|+\left\|\tilde{\mathbf{L}}_{b}-2 \Delta \mathbf{L}^{\hat{\mu}_{b}^{N}}\right\|\left\|\tilde{\mathbf{H}}_{b}\right\|^{2}\left\|\tilde{\boldsymbol{v}}_{b}\right\|\left\|\tilde{\boldsymbol{\sigma}}_{b}^{j}\right\|
$$

Now write

$$
\left.\mathcal{U}^{N}=\left\{\left\|\tilde{\mathbf{L}}_{b}-\mathbf{L}^{\hat{\mu}_{b}^{N}}\right\| \leq \epsilon_{0} \Delta,\left\|\tilde{\boldsymbol{\kappa}}_{b}-\boldsymbol{\kappa}^{\hat{\mu}_{b}^{N}}\right\|\right\} \leq \epsilon_{0} \Delta\right\}
$$

We now establish that

$$
\left\{\mathcal{J}_{N}, \tilde{\tau}_{N}>t_{b}^{(n)},\left|\beta^{7}(\boldsymbol{\alpha}, \tilde{\boldsymbol{\sigma}}, \tilde{\mathbf{G}})\right| \geq \bar{\epsilon} \Delta\right\} \subseteq \mathcal{J}_{N} \cap\left(\mathcal{U}^{N}\right)^{c} \cap\left\{\tilde{\tau}_{N}>t_{b}^{(n)}\right\}
$$

for sufficiently small $\epsilon_{0}$, and sufficiently small $\Delta$. Now $\tilde{\tau}_{N}>t_{b}^{(n)}$ implies that $\left\|\tilde{\mathbf{H}}_{b}\right\| \leq \mathfrak{c}^{-1}$, and the CauchySchwarz Inequality (and also condition $\mathcal{J}_{N}$ ) imply that $\left|v_{b}^{p q}\right|^{2} \leq N^{-1} \sum_{j \in I_{N}}\left|G^{p, j}\right|^{2} \leq 3$. This means that $\left\|\boldsymbol{v}_{b}\right\|$ is bounded. Furthermore by Jensen's Inequality $\left(N^{-1} \sum_{j \in I_{N}}\left\|\mathbf{G}_{b}^{j}\right\|\right)^{2} \leq N^{-1} \sum_{j \in I_{N}}\left\|\mathbf{G}_{b}^{j}\right\|^{2} \leq 3 M$ (as a consequence of $\left.\mathcal{J}_{N}\right)$. The probability of $\left(\mathcal{U}^{N}\right)^{c}$ is exponentially decaying, thanks to Lemma 6.7.

Lemma 6.7. For any $\epsilon_{0}>0$, there exists $n_{0} \in \mathbb{Z}^{+}$such that for all $n \geq n_{0}$, there exists $\mathfrak{n}_{0}(n)$ such that for all $\mathfrak{n} \geq \mathfrak{n}_{0}(n)$,

$$
\begin{aligned}
& \varlimsup_{N \rightarrow \infty} \sup _{1 \leq \mathfrak{q} \leq C_{\mathfrak{n}}^{N}} N^{-1} \log \mathbb{P}\left(\mathcal{J}_{N}, \tilde{\mu}^{N} \in \mathcal{V}_{\mathfrak{q}}^{N}, \sup _{0 \leq b \leq n-1} \sup _{p, q \in I_{M}}\left|\tilde{L}_{b}^{p q}-2 \Delta L_{p q}^{\hat{\mu}_{b}^{N}(\tilde{\boldsymbol{\sigma}}, \tilde{\mathbf{G}})}\right| \geq \epsilon_{0} \Delta\right)<0 \\
& \varlimsup_{N \rightarrow \infty} \sup _{1 \leq \mathfrak{q} \leq C_{\mathbf{n}}^{N}} N^{-1} \log \mathbb{P}\left(\mathcal{J}_{N}, \tilde{\mu}^{N} \in \mathcal{V}_{\mathfrak{q}}^{N}, \sup _{0 \leq b \leq n-1} \sup _{p, q \in I_{M}}\left|\tilde{\kappa}_{b}^{p q}-2 \Delta \kappa_{p q}^{\hat{\mu}_{b}^{N}(\tilde{\boldsymbol{\sigma}}, \tilde{\mathbf{G}})}\right| \geq \epsilon_{0} \Delta\right)<0 .
\end{aligned}
$$


Proof. The proofs are almost identical, so we only prove (207). Recall from (26) and (161) that

$$
\tilde{\kappa}_{b}^{i j}=N^{-1} \sum_{k=1}^{N} \tilde{G}_{b}^{j, k}\left(\tilde{\sigma}_{b}^{i, k}-\tilde{\sigma}_{b+1}^{i, k}\right) \quad \text { and } \quad \kappa_{p q}^{\hat{\mu}_{b}^{N}(\tilde{\boldsymbol{\sigma}}, \tilde{\mathbf{G}})}=N^{-1} \sum_{k=1}^{N} \tilde{G}_{b}^{j, k} \tilde{\sigma}_{b}^{i, k} c\left(\tilde{\sigma}_{b}^{i, k}, \tilde{G}_{b}^{i, k}\right) .
$$

By Lemma 3.1,

$$
\begin{aligned}
\varlimsup_{N \rightarrow \infty} \sup _{1 \leq \mathfrak{q} \leq C_{\mathfrak{n}}^{N}} N^{-1} \log \mathbb{P}\left(\mathcal{J}_{N}, \tilde{\mu}^{N} \in \mathcal{V}_{\mathfrak{q}}^{N}, \sup _{0 \leq b \leq n-1} \sup _{p, q \in I_{M}}\left|\tilde{\kappa}_{b}^{p q}-2 \Delta \kappa_{p q}^{\hat{\mu}_{b}^{N}(\tilde{\boldsymbol{\sigma}}, \tilde{\mathbf{G}})}\right| \geq \epsilon_{0} \Delta\right) \\
\quad=\sup _{0 \leq b \leq n-1} \sup _{p, q \in I_{M}} \varlimsup_{N \rightarrow \infty} \sup _{1 \leq \mathfrak{q} \leq C_{\mathfrak{n}}^{N}} N^{-1} \log \mathbb{P}\left(\mathcal{J}_{N}, \tilde{\mu}^{N} \in \mathcal{V}_{\mathfrak{q}}^{N},\left|\tilde{\kappa}_{b}^{p q}-2 \Delta \kappa_{p q}^{\hat{\mu}_{b}^{N}(\tilde{\boldsymbol{\sigma}}, \tilde{\mathbf{G}})}\right| \geq \epsilon_{0} \Delta\right) .
\end{aligned}
$$

Now a union of events bound implies that

$$
\begin{aligned}
\mathbb{P}\left(\mathcal{J}_{N}, \tilde{\mu}^{N} \in\right. & \left.\mathcal{V}_{\mathfrak{q}}^{N},\left|\tilde{\kappa}_{b}^{p q}-2 \Delta \kappa_{p q}^{\hat{\mu}_{b}^{N}(\tilde{\boldsymbol{\sigma}}, \tilde{\mathbf{G}})}\right| \geq \epsilon_{0} \Delta\right) \leq \mathbb{P}\left(\mathcal{J}_{N}, \tilde{\mu}^{N} \in \mathcal{V}_{\mathfrak{q}}^{N},\left|2 \Delta \kappa_{p q}^{\hat{\mu}_{b}^{N}\left(\tilde{\boldsymbol{\sigma}}, \tilde{\mathfrak{G}}_{\mathfrak{q}}\right)}-2 \Delta \kappa_{p q}^{\hat{\mu}_{b}^{N}(\tilde{\boldsymbol{\sigma}}, \tilde{\mathbf{G}})}\right| \geq \epsilon_{0} \Delta / 3\right) \\
& +\mathbb{P}\left(\mathcal{J}_{N}, \tilde{\mu}^{N} \in \mathcal{V}_{\mathfrak{q}}^{N},\left|\breve{\kappa}_{b}^{p q}-2 \Delta \kappa_{p q}^{\hat{\mu}_{b}^{N}\left(\tilde{\boldsymbol{\sigma}}, \tilde{\mathfrak{G}}_{\mathfrak{q}}\right)}\right| \geq \epsilon_{0} \Delta / 3\right)+\mathbb{P}\left(\mathcal{J}_{N}, \tilde{\mu}^{N} \in \mathcal{V}_{\mathfrak{q}}^{N},\left|\tilde{\kappa}_{b}^{p q}-\breve{\kappa}_{b}^{p q}\right| \geq \epsilon_{0} \Delta / 3\right) .
\end{aligned}
$$

where

$$
\breve{\kappa}_{b}^{i j}=N^{-1} \sum_{k=1}^{N} \tilde{\mathfrak{G}}_{\mathfrak{q}, t_{b}^{(n)}}^{j, k}\left(\tilde{\sigma}_{b}^{i, k}-\tilde{\sigma}_{b+1}^{i, k}\right) .
$$

Now, by definition, $\hat{\mu}^{N}\left(\tilde{\boldsymbol{\sigma}}, \tilde{\mathfrak{G}}_{\mathfrak{q}}\right) \in \mathcal{V}_{\mathfrak{q}}^{N}$. Thus if $\tilde{\mu}^{N}(\tilde{\boldsymbol{\sigma}}, \tilde{\mathbf{G}}) \in \mathcal{V}_{\mathfrak{q}}^{N}$ as well, then since the radius of the set $\mathcal{V}_{\mathfrak{q}}^{N}$ goes to zero as $\mathfrak{n} \rightarrow \infty$, (as proved in Lemma 4.1), it must be that for sufficiently large $\mathfrak{n}$

$$
\mathbb{P}\left(\tilde{\mu}^{N} \in \mathcal{V}_{\mathfrak{q}}^{N},\left|\tilde{\kappa}_{b}^{p q}-\breve{\kappa}_{b}^{p q}\right| \geq \epsilon_{0} \Delta / 3\right)=0,
$$

since

$$
\tilde{\kappa}_{b}^{p q}-\breve{\kappa}_{b}^{p q}=N^{-1} \sum_{k=1}^{N}\left\{\tilde{G}_{t_{b}^{(n)}}^{q, k}\left(\tilde{\sigma}_{b}^{p, k}-\tilde{\sigma}_{b+1}^{p, k}\right)-\tilde{\mathfrak{G}}_{\mathfrak{q}, t_{b}^{(n)}}^{q, k}\left(\tilde{\sigma}_{b}^{p, k}-\tilde{\sigma}_{b+1}^{p, k}\right)\right\} .
$$

We similarly find that for large enough $\mathfrak{n}$,

$$
\mathbb{P}\left(\mathcal{J}_{N}, \tilde{\mu}^{N} \in \mathcal{V}_{\mathfrak{q}}^{N},\left|2 \Delta \kappa_{p q}^{\hat{\mu}_{b}^{N}\left(\tilde{\boldsymbol{\sigma}}, \tilde{\mathfrak{G}}_{\mathfrak{q}}\right)}-2 \Delta \kappa_{p q}^{\hat{\mu}_{b}^{N}(\tilde{\boldsymbol{\sigma}}, \tilde{\mathbf{G}})}\right| \geq \epsilon_{0} \Delta / 3\right)=\mathbb{P}\left(\mathcal{J}_{N}, \tilde{\mu}^{N} \in \mathcal{V}_{\mathfrak{q}}^{N},\left|\kappa_{p q}^{\hat{\mu}_{b}^{N}\left(\tilde{\boldsymbol{\sigma}}, \tilde{\mathfrak{G}}_{\mathfrak{q}}\right)}-\kappa_{p q}^{\hat{\mu}_{b}^{N}(\tilde{\boldsymbol{\sigma}}, \tilde{\mathbf{G}})}\right| \geq \epsilon_{0} / 6\right)=0
$$

Concerning the other term on the right hand side of (210),

$$
\begin{aligned}
\breve{\kappa}_{b}^{p q}-2 \Delta \kappa_{p q}^{\hat{\mu}_{b}^{N}\left(\tilde{\boldsymbol{\sigma}}, \tilde{\mathfrak{G}}_{\mathfrak{q}}\right)} & =\grave{\kappa}^{p q}+\bar{\kappa}^{p q} \text { where } \\
\grave{\kappa}^{p q} & =N^{-1} \sum_{j \in I_{N}} \tilde{\mathfrak{G}}_{\mathfrak{q}, t_{b}^{q n)}}^{q, k}\left(\tilde{\sigma}_{b}^{p, k}-\tilde{\sigma}_{b+1}^{p, k}-2 \int_{t_{b}^{(n)}}^{t_{b+1}^{(n)}} \tilde{\sigma}_{s}^{p, k} c\left(\tilde{\sigma}_{b}^{p, k}, \tilde{\mathfrak{G}}_{\mathfrak{q}, t_{b}^{p(n)}}^{p, k}\right) d s\right) \\
\bar{\kappa}^{p q} & \left.=2 N^{-1} \sum_{j \in I_{N}} \tilde{\mathfrak{G}}_{\mathfrak{q}, t_{b}^{(n)}}^{q, k} \int_{t_{b}^{(n)}}^{t_{b+1}^{(n)}}\left(\tilde{\sigma}_{s}^{p, k}-\tilde{\sigma}_{b}^{p, k}\right) c\left(\tilde{\sigma}_{b}^{p, k}, \tilde{\mathfrak{G}}_{\mathfrak{q}, t_{b}^{(n)}}^{p, k}\right) d s\right)
\end{aligned}
$$

We thus find that

$$
\begin{aligned}
\mathbb{P}\left(\mathcal{J}_{N}, \tilde{\mu}^{N} \in \mathcal{V}_{\mathfrak{q}}^{N},\left|\breve{\kappa}_{b}^{p q}-2 \Delta \kappa_{p q}^{\hat{\mu}_{b}^{N}\left(\tilde{\boldsymbol{\sigma}}, \tilde{\mathfrak{G}}_{\mathfrak{q}}\right)}\right| \geq \epsilon_{0} \Delta / 3\right) \leq \\
\\
\mathbb{P}\left(\mathcal{J}_{N}, \tilde{\mu}^{N} \in \mathcal{V}_{\mathfrak{q}}^{N},\left|\grave{\kappa}^{p q}\right| \geq \epsilon_{0} \Delta / 6\right)+\mathbb{P}\left(\mathcal{J}_{N}, \tilde{\mu}^{N} \in \mathcal{V}_{\mathfrak{q}}^{N},\left|\bar{\kappa}^{p q}\right| \geq \epsilon_{0} \Delta / 6\right) .
\end{aligned}
$$

Now $\grave{\kappa}^{p q}$ is the sum of compensated Poisson Processes (which are Martingales), since, making use of the representation in (174),

$$
\tilde{\sigma}_{t}^{p, k}-\tilde{\sigma}_{b}^{p, k}=-2 \int_{t_{b}^{(n)}}^{t} \tilde{\sigma}_{s}^{p, k} d \hat{\sigma}_{s}^{p, k} \text { where } \hat{\sigma}_{s}^{p, k}=\tilde{\sigma}_{b}^{p, k} A \cdot Y_{b}^{p, k}\left(\int_{t_{b}^{(n)}}^{s} c\left(\tilde{\sigma}_{b}^{p, k}, \tilde{\mathfrak{G}}_{\mathfrak{q}, b}^{p, k}\right) d r\right)
$$


Recalling that $\left|\tilde{\mathfrak{G}}_{\mathfrak{q}, t_{b}^{(n)}}^{q, k}\right| \leq \mathfrak{n}$, it is therefore a consequence of Lemma 8.2 that

$$
\varlimsup_{N \rightarrow \infty} \sup _{1 \leq \mathfrak{q} \leq C_{\mathbf{n}}^{N}} N^{-1} \log \mathbb{P}\left(\mathcal{J}_{N}, \tilde{\mu}^{N} \in \mathcal{V}_{\mathfrak{q}}^{N},\left|\grave{\kappa}^{p q}\right| \geq \epsilon_{0} \Delta / 6\right)<0 .
$$

Since $\tilde{\sigma}_{s}^{p, k}=\tilde{\sigma}_{b}^{p, k}$ for all $s \in\left[t_{b}^{(n)}, t_{b+1}^{(n)}\right]$ if $Y_{b}^{p, k}\left(c_{1} \Delta\right)=0$, we similarly find that

$$
\begin{aligned}
& \varlimsup_{N \rightarrow \infty} \sup _{1 \leq \mathfrak{q} \leq C_{\mathfrak{n}}^{N}} N^{-1} \log \mathbb{P}\left(\mathcal{J}_{N}, \tilde{\mu}^{N} \in \mathcal{V}_{\mathfrak{q}}^{N},\left|\bar{\kappa}^{p q}\right|\right.\left.\geq \epsilon_{0} \Delta / 6\right) \\
& \leq \varlimsup_{N \rightarrow \infty} \sup _{1 \leq \mathfrak{q} \leq C_{\mathfrak{n}}^{N}} N^{-1} \log \mathbb{P}\left(4 c_{1} N^{-1} \sum_{j \in I_{N}} \chi\left\{Y_{b}^{p, k}\left(c_{1} \Delta\right)>0\right\} \geq \epsilon_{0} / 6\right)<0,
\end{aligned}
$$

for large enough $n$ (recalling that $\Delta=T n^{-1}$ ), thanks to Lemma 8.1 (ii).

Lemma 6.8. For any $\bar{\epsilon}>0$, for large enough $n \in \mathbb{Z}^{+}$,

$$
\varlimsup_{N \rightarrow \infty} N^{-1} \log \mathbb{P}\left(\tilde{\tau}_{N}>t_{b}^{(n)}, \mathcal{J}_{N},\left|\beta^{10}(\boldsymbol{\alpha}, \tilde{\boldsymbol{\sigma}}, \tilde{\mathbf{G}})\right| \geq \bar{\epsilon} \Delta\right)<0 .
$$

Proof. Since $\left|\phi_{i p}^{a}\left(\tilde{\mathbf{G}}_{b}^{j}\right)\right| \leq 1$ by definition,

$$
\begin{aligned}
\left|\beta^{10}(\boldsymbol{\alpha}, \tilde{\boldsymbol{\sigma}}, \tilde{\mathbf{G}})\right| & \leq(2 N)^{-1}\left|\sum_{j \in I_{N}} \sum_{i, p \in I_{M}} \chi\left\{\tilde{\boldsymbol{\sigma}}_{b}^{j}=\boldsymbol{\alpha}\right\} \tilde{m}_{b}^{i, j} \tilde{m}_{b}^{p, j}\right| \\
& =(2 N)^{-1} \sum_{j \in I_{N}} \chi\left\{\tilde{\boldsymbol{\sigma}}_{b}^{j}=\boldsymbol{\alpha}\right\}\left(\sum_{p \in I_{M}} \tilde{m}_{b}^{p, j}\right)^{2} \leq(2 N)^{-1} M \sum_{j \in I_{N}} \sum_{p \in I_{M}}\left(\tilde{m}_{b}^{p, j}\right)^{2},
\end{aligned}
$$

by Jensen's Inequality. Thanks to the triangle inequality,

$$
\begin{aligned}
\left\|\tilde{\mathbf{m}}_{b}^{j}\right\| & \leq\left\|\tilde{\mathbf{L}}_{b} \tilde{\mathbf{H}}_{b} \tilde{\mathbf{G}}_{b}^{j}\right\|+\mathfrak{s}\left\|\tilde{\boldsymbol{\kappa}}_{b} \tilde{\mathbf{H}}_{b} \tilde{\boldsymbol{\sigma}}_{b}^{j}\right\|+\mathfrak{s}\left\|\tilde{\mathbf{L}}_{b} \tilde{\mathbf{H}}_{b} \tilde{\boldsymbol{\nu}} \tilde{\mathbf{H}}_{b} \tilde{\boldsymbol{\sigma}}_{b}^{j}\right\| \\
& \leq\left\|\tilde{\mathbf{L}}_{b}\right\|\left\|\tilde{\mathbf{H}}_{b}\right\|\left\|\tilde{\mathbf{G}}_{b}^{j}\right\|+\mathfrak{s}\left\|\tilde{\boldsymbol{\kappa}}_{b}\right\|\left\|\tilde{\mathbf{H}}_{b}\right\|\left\|\tilde{\boldsymbol{\sigma}}_{b}^{j}\right\|+\mathfrak{s}\left\|\tilde{\mathbf{L}}_{b}\right\|\left\|\tilde{\mathbf{H}}_{b}\right\|\left\|\tilde{\boldsymbol{\nu}}_{b}\right\|\left\|\tilde{\mathbf{H}}_{b}\right\|\left\|\tilde{\boldsymbol{\sigma}}_{b}^{j}\right\| .
\end{aligned}
$$

We thus find that

$$
\left\|\tilde{\mathbf{m}}_{b}^{j}\right\|^{2} \leq 3\left\|\tilde{\mathbf{L}}_{b}\right\|^{2}\left\|\tilde{\mathbf{H}}_{b}\right\|^{2}\left\|\tilde{\mathbf{G}}_{b}^{j}\right\|^{2}+3 \mathfrak{s}^{2}\left\|\tilde{\boldsymbol{\kappa}}_{b}\right\|^{2}\left\|\tilde{\mathbf{H}}_{b}\right\|^{2}\left\|\tilde{\boldsymbol{\sigma}}_{b}^{j}\right\|^{2}+3 \mathfrak{s}^{2}\left\|\tilde{\mathbf{L}}_{b}\right\|^{2}\left\|\tilde{\mathbf{H}}_{b}\right\|^{4}\left\|\tilde{\boldsymbol{\nu}}_{b}\right\|^{2}\left\|\tilde{\boldsymbol{\sigma}}_{b}^{j}\right\|^{2} .
$$

Since $\tilde{\tau}_{N}>t_{b}^{(n)},\left\|\tilde{\mathbf{H}}_{b}\right\| \leq \mathfrak{c}^{-1}$. Since $\left|\tilde{\sigma}_{t}^{i, j}\right| \leq 1,\left\|\tilde{\boldsymbol{\sigma}}_{b}^{j}\right\| \leq \sqrt{M}$. The event $\mathcal{J}_{N}$ implies - after an application of the Cauchy-Schwarz Inequality - that $\left|\tilde{v}_{b}^{p q}\right| \leq \sqrt{3}$ and $\left|\tilde{\kappa}_{b}^{p q}\right| \leq \sqrt{3} c_{1}$. Now

$$
\begin{aligned}
& \left|\tilde{L}_{b}^{p q}\right| \leq \Delta\left(2\left|L_{p q}^{\hat{\mu}_{b}^{N}}\right|+\left|\tilde{L}_{b}^{p q}-2 \Delta L_{p q}^{\hat{\mu}_{b}^{N}(\tilde{\boldsymbol{\sigma}}, \tilde{\mathbf{G}})}\right|\right) \\
& \left|\tilde{\kappa}_{b}^{p q}\right| \leq \Delta\left(2\left|\kappa_{p q}^{\hat{\mu}_{b}^{N}}\right|+\left|\tilde{\kappa}_{b}^{p q}-2 \Delta \kappa_{p q}^{\hat{\mu}_{b}^{N}(\tilde{\boldsymbol{\sigma}}, \tilde{\mathbf{G}})}\right|\right) .
\end{aligned}
$$

Lemma 6.7 implies that the probability of the following event not holding is exponentially decaying,

$$
\left\{\sup _{p, q \in I_{M}}\left|\tilde{L}_{b}^{p q}-2 \Delta L_{p q}^{\hat{\mu}_{b}^{N}(\tilde{\boldsymbol{\sigma}}, \tilde{\mathbf{G}})}\right| \leq \Delta, \sup _{p, q \in I_{M}}\left|\tilde{\kappa}_{b}^{p q}-2 \Delta \kappa_{p q}^{\hat{\mu}_{b}^{N}(\tilde{\boldsymbol{\sigma}}, \tilde{\mathbf{G}})}\right| \leq \Delta\right\} .
$$

We can thus assume that the above events hold. Since $\left|L_{p q}^{\hat{\mu}_{b}^{N}(\tilde{\boldsymbol{\sigma}}, \tilde{\mathbf{G}})}\right| \leq c_{1}$ and

$$
\left|\kappa_{p q}^{\hat{\mu}_{b}^{N}(\tilde{\boldsymbol{\sigma}}, \tilde{\mathbf{G}})}\right| \leq c_{1} \mathbb{E}^{\hat{\mu}_{b}^{N}}\left[\left|g_{q}\right|\right] \leq c_{1} \sqrt{3},
$$

it must be that there exist positive constants $C_{1}, C_{2}$ such that

$$
\left\|\tilde{\mathbf{m}}_{b}^{j}\right\|^{2} \leq C_{1}(\Delta)^{2}\left\|\tilde{\mathbf{G}}_{b}^{j}\right\|^{2}+C_{2}(\Delta)^{2} .
$$


We thus find that

$$
N^{-1} \sum_{j \in I_{N}}\left\|\tilde{\mathbf{m}}_{b}^{j}\right\|^{2} \leq C_{1}(\Delta)^{2} \sum_{j \in I_{N}}\left\|\tilde{\mathbf{G}}_{b}^{j}\right\|^{2}+C_{2}(\Delta)^{2} \leq 3 C_{1} M(\Delta)^{2}+C_{2}(\Delta)^{2}
$$

as long as the event $\mathcal{J}_{N}$ holds. In conclusion, as long as the events $\tilde{\tau}_{N} \geq t_{b}^{(n)}, \mathcal{J}_{N}$ and (215) hold, it must be that

$$
\left|\beta^{10}(\boldsymbol{\alpha}, \tilde{\boldsymbol{\sigma}}, \tilde{\mathbf{G}})\right| \leq 3 C_{1} M(\Delta)^{2}+C_{2}(\Delta)^{2} .
$$

Clearly for small enough $\Delta,(213)$ must hold.

\section{Using the Gaussian Law to Estimate the Field Dynamics}

In this section we continue the proof of Lemma 5.4: providing bounds for the terms $\beta^{6}(\boldsymbol{\alpha}, \tilde{\boldsymbol{\sigma}}, \tilde{\mathbf{G}}), \beta^{8}(\boldsymbol{\alpha}, \tilde{\boldsymbol{\sigma}}, \tilde{\mathbf{G}}), \beta^{9}(\boldsymbol{\alpha}, \tilde{\boldsymbol{\sigma}}, \tilde{\mathbf{G}})$. The bounding of these terms requires the law $\gamma$ of the Gaussian connections $\left\{J^{j k}\right\}_{j, k \in I_{N}}$. Recall that the processes $\left\{\tilde{\boldsymbol{\sigma}}_{i}\right\}_{1 \leq i \leq C_{\mathfrak{n}}^{N}}$ are independent of the connections, and so conditioning on these processes does not affect the distribution of the connections. For fixed $\tilde{\boldsymbol{\sigma}}_{b} \in \mathcal{E}^{M N}$ and any $\mathbf{g} \in \mathbb{R}^{M N}$, let $\gamma_{\tilde{\boldsymbol{\sigma}}_{b}, \mathbf{g}} \in \mathcal{M}_{1}^{+}\left(\mathbb{R}^{N^{2}}\right)$ be the regular conditional probability distribution of the connections $\mathbf{J}$, conditionally on

$$
N^{-1 / 2} \sum_{k \in I_{N}} J^{j k} \tilde{\sigma}_{b}^{p, k}=g^{p, j} .
$$

Standard theory dictates that $\gamma_{\tilde{\boldsymbol{\sigma}}_{b}, \mathbf{g}}$ is Gaussian (see for instance Theorem A.1.3 in [50]). We start by determining expressions for the conditional mean and variance of $\gamma_{\tilde{\boldsymbol{\sigma}}_{b}, \mathbf{g}}$ in Section 7.1. We then use these expressions to bound $\beta^{6}, \beta^{8}$ and $\beta^{9}$ in Section 7.2.

\subsection{The Conditional Mean and Covariance}

The main result of this section is Lemma 7.3: this lemma is crucial because it demonstrates that the conditional mean of the increment $\tilde{G}_{b+1}^{i, j}-\tilde{G}_{b}^{i, j}$ can be written as a function of the variables $\left\{\tilde{\boldsymbol{\sigma}}_{b}^{j}, \tilde{\boldsymbol{\sigma}}_{b+1}^{j}, \tilde{\mathbf{G}}_{b}^{j}\right\}$ and the empirical measure at time $t_{b}^{(n)}$, i.e. $\hat{\mu}^{N}\left(\tilde{\boldsymbol{\sigma}}_{b}, \tilde{\mathbf{G}}_{b}\right)$. This property allows us to obtain a closed expression for the dynamics of the empirical process. We also determine some bounds on the conditional variance matrix.

We write

$$
\tilde{G}_{b}^{i, j}=N^{-\frac{1}{2}} \sum_{k=1}^{N} J^{j k} \tilde{\sigma}_{b}^{i, k}, \tilde{F}_{b}^{i, j}=N^{-\frac{1}{2}} \sum_{k=1}^{N} J^{j k}\left(\tilde{\sigma}_{b+1}^{i, k}-\tilde{\sigma}_{b}^{i, k}\right)
$$

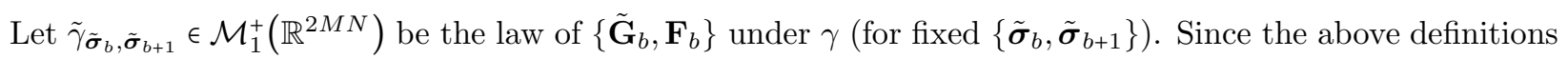
are linear, standard theory dictates that $\tilde{\gamma}_{\tilde{\boldsymbol{\sigma}}_{b}, \tilde{\boldsymbol{\sigma}}_{b+1}}$ is Gaussian. Next define $\gamma_{\tilde{\boldsymbol{\sigma}}_{b}, \tilde{\boldsymbol{\sigma}}_{b+1}, \tilde{\mathbf{G}}_{b}}^{N} \in \mathcal{M}_{1}^{+}\left(\mathbb{R}^{M N}\right)$ to be the law of $\mathbf{F}_{b}$ under $\tilde{\gamma}_{\tilde{\boldsymbol{\sigma}}_{b}, \tilde{\boldsymbol{\sigma}}_{b+1}}$, conditionally on $\tilde{\mathbf{G}}_{b}$. The rest of this section is devoted to finding tractable

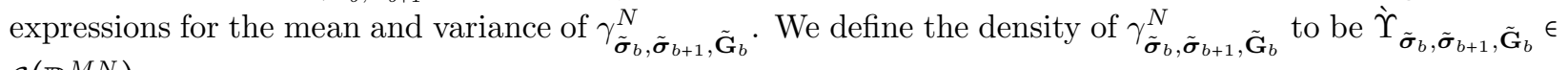
$\mathcal{C}\left(\mathbb{R}^{M N}\right)$.

Let $\Upsilon_{\tilde{\boldsymbol{\sigma}}_{b}, \tilde{\boldsymbol{\sigma}}_{b+1}}^{N} \in \mathcal{C}\left(\mathbb{R}^{2 M N}\right)$ be the Gaussian density of $\left\{\tilde{G}_{b}^{i, j}, \tilde{F}_{b+1}^{i, j}\right\}_{j=1}^{N}$ under $\gamma$, i.e.

$$
\Upsilon_{\tilde{\boldsymbol{\sigma}}_{b}, \tilde{\boldsymbol{\sigma}}_{b+1}}^{N}\left(\tilde{\mathbf{G}}_{b}, \tilde{\mathbf{F}}_{b}\right)=(2 \pi)^{-N}\left(\operatorname{det}\left(\mathcal{L}_{N}\right)\right)^{-\frac{1}{2}} \exp \left(-\left(\tilde{\mathbf{G}}_{b}, \tilde{\mathbf{F}}_{b}\right)^{T} \overline{\mathcal{K}}_{N}\left(\tilde{\boldsymbol{\sigma}}_{b}, \tilde{\boldsymbol{\sigma}}_{b+1}\right)^{-1}\left(\tilde{\mathbf{G}}_{b}, \tilde{\mathbf{F}}_{b}\right) / 2\right),
$$

and $\overline{\mathcal{K}}_{N}\left(\tilde{\boldsymbol{\sigma}}_{b}, \tilde{\boldsymbol{\sigma}}_{b+1}\right)$ is the $2 N M \times 2 N M$ covariance matrix of $\left\{\tilde{G}_{b}^{i, j}, \tilde{F}_{b}^{i, j}\right\}_{i \in I_{N}, j \in I_{N}}$, i.e.

$$
\overline{\mathcal{K}}_{N}=\left(\begin{array}{cc}
\mathcal{K}_{N}\left(\tilde{\boldsymbol{\sigma}}_{b}\right) & \grave{\mathcal{K}}_{N}\left(\tilde{\boldsymbol{\sigma}}_{b}, \tilde{\boldsymbol{\sigma}}_{b+1}\right) \\
\left(\grave{\mathcal{K}}_{N}\right)\left(\tilde{\boldsymbol{\sigma}}_{b}, \tilde{\boldsymbol{\sigma}}_{b+1}\right)^{T} & \tilde{\mathcal{K}}_{N}\left(\tilde{\boldsymbol{\sigma}}_{b+1}\right)
\end{array}\right) .
$$


The contents of $\overline{\mathcal{K}}_{N}\left(\tilde{\boldsymbol{\sigma}}_{b}, \tilde{\boldsymbol{\sigma}}_{b+1}\right)$ are the following $M N \times M N$ square matrices, with the replica indices at the top, and the spin indices at the bottom, i.e. for $i, m \in I_{M}$ and $j, k \in I_{N}$,

$$
\begin{gathered}
\mathcal{K}_{N}\left(\tilde{\boldsymbol{\sigma}}_{b}\right)_{j k}^{i m}=\mathbb{E}^{\gamma}\left[\tilde{G}_{b}^{i, j} \tilde{G}_{b}^{m, k}\right]=\delta(j, k) N^{-1} \sum_{l=1}^{N} \tilde{\sigma}_{b}^{i, l} \tilde{\sigma}_{b}^{m, l}+\frac{\mathfrak{s}}{N} \tilde{\sigma}_{b}^{m, j} \tilde{\sigma}_{b}^{i, k} \\
\tilde{\mathcal{K}}_{N}\left(\tilde{\boldsymbol{\sigma}}_{b}, \tilde{\boldsymbol{\sigma}}_{b+1}\right)_{j k}^{i m}=\mathbb{E}^{\gamma}\left[\tilde{F}_{b}^{i, j} \tilde{F}_{b}^{m, k}\right]=\delta(j, k) N^{-1} \sum_{l=1}^{N}\left(\tilde{\sigma}_{b+1}^{i, l}-\tilde{\sigma}_{b}^{i, l}\right)\left(\tilde{\sigma}_{b+1}^{m, l}-\tilde{\sigma}_{b}^{m, l}\right)+\frac{\mathfrak{s}}{N}\left(\tilde{\sigma}_{b+1}^{m, j}-\tilde{\sigma}_{b}^{m, j}\right)\left(\tilde{\sigma}_{b+1}^{i, k}-\tilde{\sigma}_{b}^{i, k}\right) \\
\grave{\mathcal{K}}_{N}\left(\tilde{\boldsymbol{\sigma}}_{b}, \tilde{\boldsymbol{\sigma}}_{b+1}\right)_{j k}^{i m}=\mathbb{E}^{\gamma}\left[\tilde{F}_{b}^{i, j} \tilde{G}_{b}^{m, k}\right]=\delta(j, k) N^{-1} \sum_{l=1}^{N}\left(\tilde{\sigma}_{b+1}^{i, l}-\tilde{\sigma}_{b}^{i, l}\right) \tilde{\sigma}_{b}^{m, l}+\frac{\mathfrak{s}}{N}\left(\tilde{\sigma}_{b+1}^{i, k}-\tilde{\sigma}_{b}^{i, k}\right) \tilde{\sigma}_{b}^{m, j}
\end{gathered}
$$

Standard theory (see for instance Theorem A.1.3 in [50]) dictates that the density $\grave{\Upsilon}_{\tilde{\boldsymbol{\sigma}}_{b} \tilde{\boldsymbol{\sigma}}_{b+1}, \tilde{\mathbf{G}}_{b}}^{N}\left(\tilde{\mathbf{F}}_{b}\right)$ of $\gamma_{\tilde{\boldsymbol{\sigma}}_{b}, \tilde{\sigma}_{b+1}, \tilde{\mathbf{G}}_{b}}$ assumes the form

$$
\begin{aligned}
\grave{\Upsilon}_{\tilde{\boldsymbol{\sigma}}_{b}}^{N} \tilde{\boldsymbol{\sigma}}_{b+1}, \tilde{\mathbf{G}}_{b}\left(\tilde{\mathbf{F}}_{b}\right)=(2 \pi)^{-N / 2} \operatorname{det}\left(\mathcal{R}^{N}\left(\tilde{\boldsymbol{\sigma}}_{b}, \tilde{\boldsymbol{\sigma}}_{b+1}\right)\right)^{-\frac{1}{2}} & \\
& \exp \left(-\frac{1}{2}\left\{\tilde{\mathbf{F}}_{b}-\tilde{\mathbf{m}}_{b}\left(\tilde{\boldsymbol{\sigma}}_{b}, \tilde{\boldsymbol{\sigma}}_{b+1}, \tilde{\mathbf{G}}_{b}\right)\right\}^{T} \mathcal{R}^{N}\left(\tilde{\boldsymbol{\sigma}}_{b}, \tilde{\boldsymbol{\sigma}}_{b+1}\right)^{-1}\left\{\tilde{\mathbf{F}}_{b}-\tilde{\mathbf{m}}_{b}\left(\tilde{\boldsymbol{\sigma}}_{b}, \tilde{\boldsymbol{\sigma}}_{b+1}, \tilde{\mathbf{G}}_{b}\right)\right\}\right) .
\end{aligned}
$$

Here $\tilde{\mathbf{m}}_{b}\left(\tilde{\boldsymbol{\sigma}}_{b}, \tilde{\boldsymbol{\sigma}}_{b+1}, \tilde{\mathbf{G}}_{b}\right):=\left\{\tilde{m}_{b}^{i, j}\left(\tilde{\boldsymbol{\sigma}}_{b}, \tilde{\boldsymbol{\sigma}}_{b+1}, \tilde{\mathbf{G}}_{b}\right)\right\}_{(i, j) \in I_{M, N}}$ is the vector of conditional means of $\left\{F_{b+1}^{i, j}\right\}$ i.e.

$$
\tilde{m}_{b}^{i, j}\left(\tilde{\boldsymbol{\sigma}}_{b}, \tilde{\boldsymbol{\sigma}}_{b+1}, \tilde{\mathbf{G}}_{b}\right)=\left(\grave{\mathcal{K}}_{N}\left(\tilde{\boldsymbol{\sigma}}_{b}, \tilde{\boldsymbol{\sigma}}_{b+1}\right) \mathcal{K}_{N}\left(\tilde{\boldsymbol{\sigma}}_{b}\right)^{-1} \tilde{\mathbf{G}}_{b}\right)^{i, j},
$$

i.e. in the above $\tilde{m}_{b}^{i, j}$ is the element with index $(i, j)$ in the above vector resulting from two matrix multiplications on the vector $\tilde{\mathbf{G}}_{b}$. $\mathcal{R}_{N}\left(\tilde{\boldsymbol{\sigma}}_{b}, \tilde{\boldsymbol{\sigma}}_{b+1}\right)$ is the $M N \times M N$ conditional covariance matrix of $\tilde{\mathbf{F}}_{b}$, i.e.

$$
\begin{aligned}
& \mathcal{R}_{N}\left(\tilde{\boldsymbol{\sigma}}_{b}, \tilde{\boldsymbol{\sigma}}_{b+1}\right)=\tilde{\mathcal{K}}_{N}\left(\tilde{\boldsymbol{\sigma}}_{b}, \tilde{\boldsymbol{\sigma}}_{b+1}\right)-\mathcal{L}_{N}\left(\tilde{\boldsymbol{\sigma}}_{b}, \tilde{\boldsymbol{\sigma}}_{b+1}\right) \text { where } \\
& \mathcal{L}_{N}\left(\tilde{\boldsymbol{\sigma}}_{b}, \tilde{\boldsymbol{\sigma}}_{b+1}\right)=\grave{\mathcal{K}}_{N}\left(\tilde{\boldsymbol{\sigma}}_{b}, \tilde{\boldsymbol{\sigma}}_{b+1}\right) \mathcal{K}_{N}\left(\tilde{\boldsymbol{\sigma}}_{b}\right)^{-1} \grave{\mathcal{K}}_{N}\left(\tilde{\boldsymbol{\sigma}}_{b}, \tilde{\boldsymbol{\sigma}}_{b+1}\right)^{T}
\end{aligned}
$$

noting that $\mathcal{L}_{N}$ is an $M N \times M N$ matrix.

Lemma 7.1. Recall that $\|\cdot\|$ is the operator norm and the definition of $\tilde{\mathbf{L}}_{b}$ in (160). We have the following bounds on $N M \times N M$ square matrices

$$
\begin{aligned}
\left\|\mathcal{R}_{N}\left(\tilde{\boldsymbol{\sigma}}_{b}, \tilde{\boldsymbol{\sigma}}_{b+1}\right)\right\| & \leq\left\|\tilde{\mathcal{K}}\left(\tilde{\boldsymbol{\sigma}}_{b}, \tilde{\boldsymbol{\sigma}}_{b+1}\right)\right\| \\
\left\|\tilde{\mathcal{K}}\left(\tilde{\boldsymbol{\sigma}}_{b}, \tilde{\boldsymbol{\sigma}}_{b+1}\right)\right\| & \leq N^{-1}\{4 M+4 \mathfrak{s} M\} \sup _{i \in I_{M}} \sum_{l=1}^{N} \chi\left\{\tilde{\sigma}_{b+1}^{i, l} \neq \tilde{\sigma}_{b}^{i, l}\right\} \\
\left\|\dot{\mathcal{K}}\left(\tilde{\boldsymbol{\sigma}}_{b}, \tilde{\boldsymbol{\sigma}}_{b+1}\right)\right\| & \leq\left\|\tilde{L}_{b}\right\|+\sqrt{2} N^{-1 / 2} \\
\left\|\mathcal{K}\left(\tilde{\boldsymbol{\sigma}}_{b}\right)\right\| & \leq M\{1+\mathfrak{s}\}
\end{aligned}
$$

Proof. (228) is a known property of finite Gaussian systems: the conditional variance is always less than or equal to the variance. It follows from the fact that $\mathcal{R}_{N}, \tilde{\mathcal{K}}_{N}$ and $\mathcal{L}_{N}$ are positive definite.

For (229), write $U_{b}^{i}=N^{-1} \sum_{l=1}^{N} \chi\left\{\tilde{\sigma}_{b+1}^{i, l} \neq \tilde{\sigma}_{b}^{i, l}\right\}$ and $\mathfrak{a}=\left(\mathfrak{a}^{i, j}\right)_{i \in I_{M}, j \in I_{N}}$. Observe that

$$
\begin{aligned}
\sum_{i, m \in I_{M}} & \sum_{j, k \in I_{N}} \tilde{\mathcal{K}}_{N}\left(\tilde{\boldsymbol{\sigma}}_{b}, \tilde{\boldsymbol{\sigma}}_{b+1}\right)_{j k}^{i m} \mathfrak{a}^{i, j} \mathfrak{a}^{m, k} \\
\quad & N^{-1} \sum_{l, j \in I_{N}} \sum_{i, m \in I_{M}} \mathfrak{a}^{i, j} \mathfrak{a}^{m, j}\left(\tilde{\sigma}_{b+1}^{i, l}-\tilde{\sigma}_{b}^{i, l}\right)\left(\tilde{\sigma}_{b+1}^{m, l}-\tilde{\sigma}_{b}^{m, l}\right)+\frac{\mathfrak{s}}{N} \sum_{i \in I_{M}, j, k \in I_{N}} \mathfrak{a}^{i, j} \mathfrak{a}^{m, k}\left(\tilde{\sigma}_{b+1}^{m, j}-\tilde{\sigma}_{b}^{m, j}\right)\left(\tilde{\sigma}_{b+1}^{i, k}-\tilde{\sigma}_{b}^{i, k}\right) \\
\quad \leq & 4 \sum_{i, m \in I_{M}}\left\{\sum_{j \in I_{N}}\left|\mathfrak{a}^{i, j}\right|^{2} \sum_{k \in I_{N}}\left|\mathfrak{a}^{m, k}\right|^{2} U_{b}^{i} U_{b}^{m}\right\}^{\frac{1}{2}}+4 \mathfrak{s} \sum_{i, m \in I_{M}}\left\{U_{b}^{i} U_{b}^{m} \sum_{j \in I_{N}}\left(\mathfrak{a}^{i, j}\right)^{2} \sum_{k \in I_{N}}\left(\mathfrak{a}^{m, k}\right)^{2}\right\}^{\frac{1}{2}}
\end{aligned}
$$


using the Cauchy-Schwarz Inequality, and the fact that since $\left|\sigma_{b+1}^{i, l}-\sigma_{b}^{i, l}\right| \leq 2$,

$$
N^{-1} \sum_{l=1}^{N}\left(\sigma_{b+1}^{i, l}-\sigma_{b}^{i, l}\right)^{2} \leq 4 U_{b}^{i}
$$

Now

$$
\sum_{i, m \in I_{M}}\left\{\sum_{j \in I_{N}}\left(\mathfrak{a}^{i, j}\right)^{2} \sum_{k \in I_{N}}\left(\mathfrak{a}^{m, k}\right)^{2}\right\}^{\frac{1}{2}}=\left(\sum_{i \in I_{M}}\left\{\sum_{j \in I_{N}}\left(\mathfrak{a}^{i, j}\right)^{2}\right\}^{\frac{1}{2}}\right)^{2} \leq M \sum_{i \in I_{M}} \sum_{j \in I_{N}}\left(\mathfrak{a}^{i, j}\right)^{2}
$$

by the (discrete) Jensen's Inequality. We thus find that

$$
\sum_{i, m \in I_{M}} \sum_{j, k \in I_{N}} \tilde{\mathcal{K}}_{N}\left(\tilde{\boldsymbol{\sigma}}_{b}, \tilde{\boldsymbol{\sigma}}_{b+1}\right)_{j k}^{i m} \mathfrak{a}^{i, j} \mathfrak{a}^{m, k} \leq 4 M(1+\mathfrak{s}) \sup _{i \in I_{M}} U_{b}^{i} \sum_{p \in I_{M}} \sum_{j \in I_{N}}\left(\mathfrak{a}^{p, j}\right)^{2} .
$$

which implies (ii). The proofs of (230) and (231) are analogous to the proof of (ii) and are neglected.

Recall that the $M \times M$ matrices $\left\{\tilde{\mathbf{K}}_{b}, \tilde{\mathbf{L}}_{b}, \tilde{\boldsymbol{\kappa}}_{b}, \tilde{\boldsymbol{v}}_{b}\right\}$ were defined to have the following elements

$$
\begin{aligned}
& \tilde{K}_{b}^{i j}=N^{-1} \sum_{l=1}^{N} \tilde{\sigma}_{b}^{i, l} \tilde{\sigma}_{b}^{j, l}, \tilde{L}_{b}^{i j}=N^{-1} \sum_{k=1}^{N} \tilde{\sigma}_{b}^{j, k}\left(\tilde{\sigma}_{b}^{i, k}-\tilde{\sigma}_{b+1}^{i, k}\right) \\
& \tilde{\kappa}_{b}^{i j}=N^{-1} \sum_{k=1}^{N} \tilde{G}_{b}^{j, k}\left(\tilde{\sigma}_{b}^{i, k}-\tilde{\sigma}_{b+1}^{i, k}\right), \tilde{v}_{b}^{i j}=N^{-1} \sum_{k=1}^{N} \tilde{\sigma}_{b}^{i, k} \tilde{G}_{b}^{j, k} .
\end{aligned}
$$

The following lemma is useful because it implies that the covariance matrix of the fields $\left\{\tilde{G}_{b}^{p, j}\right\}_{p \in I_{M}, j \in I_{N}}$ is nonsingular whenever $\tilde{\mathbf{K}}_{b}$ is nonsingular.

Lemma 7.2. $\left\|\mathcal{K}_{N}\left(\tilde{\boldsymbol{\sigma}}_{b}\right)\right\| \geq\left\|\tilde{\mathbf{K}}_{b}\right\|$ and $\left\|\mathcal{K}_{N}\left(\tilde{\boldsymbol{\sigma}}_{b}\right)^{-1}\right\| \leq\left\|\tilde{\mathbf{K}}_{b}^{-1}\right\|$

Proof. Making use of double index notation, define the $M^{2} \times N M$ matrix $Q$ to have elements, for $u, v, q \in I_{M}$ and $k \in I_{N}$,

$$
Q_{u v, q k}=N^{-1 / 2} x^{v, k} \delta(q, u) .
$$

Write $\mathcal{U}_{N}$ to be the $N M \times N M$ block diagonal matrix, with each $M \times M$ diagonal block equal to $\tilde{\mathbf{K}}_{b}$. We then find that

$$
\mathcal{K}_{N}\left(\tilde{\boldsymbol{\sigma}}_{b}\right)=\mathcal{U}_{N}+\mathfrak{s} Q^{T} Q .
$$

It is thus clear that $\left\|\mathcal{K}_{N}\left(\tilde{\boldsymbol{\sigma}}_{b}\right)\right\| \geq\left\|\mathcal{U}_{N}\right\|=\left\|\tilde{\mathbf{K}}_{b}\right\|$. The second identity in the lemma follows from the fact that the smallest eigenvalue of $\left\|\mathcal{K}_{N}\left(\tilde{\boldsymbol{\sigma}}_{b}\right)\right\|$ must be greater than or equal to the smallest eigenvalue of $\tilde{\mathbf{K}}_{b}$.

We now determine a precise expression for the conditional mean. It is fundamental to the entire paper that $\tilde{\mathbf{m}}^{j}$ can be written as a function purely of (i) 'local variables' (i.e. $\tilde{\mathbf{G}}_{b}^{j}$, $\tilde{\boldsymbol{\sigma}}_{b}^{j}$ and $\tilde{\boldsymbol{\sigma}}_{b+1}^{j}$, and (ii) the empirical measure (i.e. via the definitions in (232) - (233)).

Lemma 7.3. Assume that $\tilde{\boldsymbol{\sigma}}_{b} \in \mathcal{X}^{N}$. (i) $\tilde{\mathbf{K}}_{b}$ is invertible, and we write $\tilde{\mathbf{H}}_{b}=\tilde{\mathbf{K}}_{b}^{-1}$. $\left\|\tilde{\mathbf{H}}_{b}\right\| \leq \mathfrak{c}^{-1}$.

(ii) Writing $\tilde{\boldsymbol{\sigma}}_{b}^{j}=\left(\sigma_{b}^{1, j}, \ldots, \sigma_{b}^{M, j}\right)^{T}, \tilde{\mathbf{G}}_{b}^{j}=\left(\tilde{G}_{b}^{1, j}, \ldots, \tilde{G}_{b}^{M, j}\right)^{T}$ and $\tilde{\mathbf{m}}_{b}^{j}=\left(\tilde{m}_{b}^{1, j}, \ldots, \tilde{m}_{b}^{M, j}\right)^{T}$, we have that

$$
\tilde{\mathbf{m}}_{b}^{j}=-\tilde{\mathbf{L}}_{b} \tilde{\mathbf{H}}_{b} \tilde{\mathbf{G}}_{b}^{j}-\mathfrak{s} \tilde{\boldsymbol{\kappa}}_{b} \tilde{\mathbf{H}}_{b} \tilde{\boldsymbol{\sigma}}_{b}^{j}+\mathfrak{s} \tilde{\mathbf{L}}_{b} \tilde{\mathbf{H}}_{b} \tilde{\boldsymbol{v}}_{b} \tilde{\mathbf{H}}_{b} \tilde{\boldsymbol{\sigma}}_{b}^{j} .
$$

Proof. The fact that $\tilde{\boldsymbol{\sigma}}_{b} \in \mathcal{X}^{N}$ implies that the $M \times M$ square matrix $\tilde{\mathbf{K}}_{b}$ (with elements defined in (232)) has eigenvalues greater than $\mathfrak{c}$. Since it is co-diagonal with its inverse, it must be that $\left\|\tilde{\mathbf{H}}_{b}\right\| \leq \mathfrak{c}^{-1}$. It then follows from Lemma 7.2 that $\mathcal{K}_{N}\left(\tilde{\boldsymbol{\sigma}}_{b}\right)$ must also be invertible. Let $\mathbf{V}=\mathcal{K}_{N}\left(\tilde{\boldsymbol{\sigma}}_{b}\right)^{-1} \tilde{\mathbf{G}}_{b}$. Writing $\mathbf{V}=\left\{V^{i, j}\right\}_{i \in I_{M}, j \in I_{N}}$, it must be that

$$
\tilde{G}_{b}^{i, j}=\sum_{k \in I_{N}, m \in I_{M}} \mathbb{E}^{\gamma}\left[\tilde{G}_{b}^{i, j} \tilde{G}_{b}^{m, k}\right] V^{m, k}
$$


Substituting the identity in $(221)$ we find that

$$
\tilde{G}_{b}^{i, j}=\sum_{m \in I_{M}}\left\{\tilde{K}_{b}^{i m} V^{m, j}+\mathfrak{s} N^{-1} \sum_{k \in I_{N}, m \in I_{M}} \tilde{\sigma}_{b}^{m, j} \tilde{\sigma}_{b}^{i, k} V^{m, k}\right\} .
$$

Rearranging (238), we find that

$$
\begin{aligned}
V^{i, j} & =\sum_{m \in I_{M}} \tilde{H}_{b}^{i m}\left\{G_{b}^{m, j}-\frac{\mathfrak{s}}{N} \sum_{k \in I_{N}, p \in I_{M}} \tilde{\sigma}_{b}^{p, j} \tilde{\sigma}_{b}^{m, k} V^{p, k}\right\} \\
& =\sum_{m \in I_{M}} \tilde{H}_{b}^{i m}\left\{G_{b}^{m, j}-\mathfrak{s} \sum_{p \in I_{M}} Q^{m p} \tilde{\sigma}_{b}^{p, j}\right\}
\end{aligned}
$$

where

$$
Q^{m p}=N^{-1} \sum_{k \in I_{N}} \tilde{\sigma}_{b}^{m, k} V^{p, k}
$$

In matrix / vector notation, this means that $\mathbf{V}^{j}=\tilde{\mathbf{H}} \tilde{\mathbf{G}}_{b}^{j}-\mathfrak{s} \tilde{\mathbf{H}} \mathbf{Q} \tilde{\boldsymbol{\sigma}}_{b}^{j}$. Now using the identities in (223) and (225),

$$
\begin{aligned}
\tilde{\mathbf{m}}_{b}^{j} & =-\tilde{\mathbf{L}}_{b}\left(\tilde{\mathbf{H}}_{b} \tilde{\mathbf{G}}_{b}^{j}-\mathfrak{s} \tilde{\mathbf{H}}_{b} \mathbf{Q} \tilde{\boldsymbol{\sigma}}_{b}^{j}\right)-\mathfrak{s} \tilde{\boldsymbol{\kappa}}_{b} \tilde{\mathbf{H}}_{\boldsymbol{\boldsymbol { \sigma }}_{b}^{j}}+\mathfrak{s}^{2} \tilde{\mathbf{L}}_{b} \mathbf{Q}^{T} \tilde{\mathbf{H}}_{b} \tilde{\boldsymbol{\sigma}}_{b}^{j} \\
& =-\tilde{\mathbf{L}}_{b} \tilde{\mathbf{H}}_{b} \tilde{\mathbf{G}}_{b}^{j}-\mathfrak{s} \tilde{\boldsymbol{\kappa}}_{b} \tilde{\mathbf{H}}_{b} \tilde{\boldsymbol{\sigma}}_{b}^{j}+\mathfrak{s} \tilde{\mathbf{L}}_{b}\left(\tilde{\mathbf{H}}_{b} \mathbf{Q}+\mathfrak{s} \mathbf{Q}^{T} \tilde{\mathbf{H}}_{b}\right) \tilde{\boldsymbol{\sigma}}_{b}^{j}
\end{aligned}
$$

We add $\tilde{\sigma}_{b}^{p, j}$ to both sides of (238), and sum over $j$, obtaining that

$$
\tilde{\boldsymbol{\nu}}=\mathbf{Q} \tilde{\mathbf{K}}_{b}+\mathfrak{s} \tilde{\mathbf{K}}_{b} \mathbf{Q}^{T}
$$

Multiplying both sides of the above equation by $\tilde{\mathbf{H}}_{b}$, we find that

$$
\tilde{\mathbf{H}}_{b} \tilde{\boldsymbol{\nu}}_{b} \tilde{\mathbf{H}}_{b}=\tilde{\mathbf{H}}_{b} \tilde{\mathbf{Q}}+\mathfrak{s} \tilde{\mathbf{Q}}^{T} \tilde{\mathbf{H}}_{b} .
$$

Substituting this into (240), we find that, as required,

$$
\tilde{\mathbf{m}}_{b}^{j}=-\tilde{\mathbf{L}}_{b} \tilde{\mathbf{H}}_{b} \tilde{\mathbf{G}}_{b}^{j}-\mathfrak{s} \tilde{\boldsymbol{\kappa}}_{b} \tilde{\mathbf{H}}_{b} \tilde{\boldsymbol{\sigma}}_{b}^{j}+\mathfrak{s} \tilde{\mathbf{L}}_{b} \tilde{\mathbf{H}}_{b} \tilde{\boldsymbol{\nu}}_{b} \tilde{\mathbf{H}}_{b} \tilde{\boldsymbol{\sigma}}_{b}^{j}
$$

\subsection{Bounding $\beta^{6}, \beta^{8}, \beta^{9}$}

These terms are defined in (164), (166) and (167). $\beta^{6}$ and $\beta^{8}$ concern the linear increments in the fields $\tilde{G}_{b+1}^{q, j}-\tilde{G}_{n}^{q, j}$, and $\beta^{9}$ concerns the quadratic increments in the fields.

Lemma 7.4. For any $\bar{\epsilon}>0$, for all large enough $n$,

$$
\begin{aligned}
\sup _{\boldsymbol{\alpha} \in \mathcal{E}^{M}} \sup _{0 \leq b<n} \varlimsup_{N \rightarrow \infty} \sup _{1 \leq \mathfrak{q} \leq C_{\mathfrak{n}}^{N}} N^{-1} \log \mathbb{P}\left(\mathcal{J}_{N}, \tilde{\mu}^{N} \in \mathcal{V}_{\mathfrak{q}}^{N}, \tilde{\tau}_{N}>t_{b}^{(n)},\left|\beta^{6}(\boldsymbol{\alpha}, \tilde{\boldsymbol{\sigma}}, \tilde{\mathbf{G}})\right| \geq \bar{\epsilon} \Delta\right)<0 \\
\sup _{\boldsymbol{\alpha} \in \mathcal{E}^{M}} \sup _{0 \leq b<n} \varlimsup_{N \rightarrow \infty} \sup _{1 \leq \mathfrak{q} \leq C_{\mathfrak{n}}^{N}} N^{-1} \log \mathbb{P}\left(\mathcal{J}_{N}, \tilde{\mu}^{N} \in \mathcal{V}_{\mathfrak{q}}^{N}, \tilde{\tau}_{N}>t_{b}^{(n)},\left|\beta^{8}(\boldsymbol{\alpha}, \tilde{\boldsymbol{\sigma}}, \tilde{\mathbf{G}})\right| \geq \bar{\epsilon} \Delta\right)<0 .
\end{aligned}
$$

Proof. The proofs of the above two terms are very similar, thus we only prove (244).

Define $R_{\mathfrak{q}, \tilde{\boldsymbol{\sigma}}_{b}}^{N} \in \mathcal{M}_{1}^{+}\left(\mathcal{D}\left(\left[t_{b}^{(n)}, T\right], \mathcal{E}^{M}\right)^{N}\right)$ to be the law of the stochastic process $\tilde{\boldsymbol{\sigma}}_{\mathfrak{q}, t}$, conditioned on its value $\tilde{\boldsymbol{\sigma}}_{b}$ at time $t_{b}^{(n)}$. (Recall the definition of this process in Section 4.2). As previously, we drop the subscript and write $\tilde{\boldsymbol{\sigma}}_{\mathfrak{q}, t}:=\tilde{\boldsymbol{\sigma}}_{t}$. Define $Q_{\tilde{\boldsymbol{\sigma}}_{b}, \tilde{\mathbf{G}}_{b}}^{N}$ to be the regular conditional probability distribution of $\left(\mathbf{J}, \tilde{\boldsymbol{\sigma}}_{b}\right)$, conditionally on both $\tilde{\boldsymbol{\sigma}}_{b}$ and $\tilde{\mathbf{G}}_{b}$. Since $\tilde{\boldsymbol{\sigma}}_{b}$ and $\mathbf{J}$ are independent, we have that

$$
Q_{\tilde{\boldsymbol{\sigma}}_{b}, \tilde{\mathbf{G}}_{b}}^{N}=\gamma_{\tilde{\boldsymbol{\sigma}}_{b}, \tilde{\mathbf{G}}_{b}} \otimes R_{\mathfrak{q}, \tilde{\boldsymbol{\sigma}}_{b}}^{N}
$$


Writing $\mathcal{Z}^{N}=\left\{\mathbf{g} \in \mathbb{R}^{M N}: \sup _{i \in I_{M}} \sum_{j \in I_{N}}\left|g^{i, j}\right|^{2} \leq 3 N\right\}$, this means that

$$
\mathbb{P}\left(\mathcal{J}_{N} \text { and }\left|\beta^{6}(\boldsymbol{\alpha}, \tilde{\boldsymbol{\sigma}}, \tilde{\mathbf{G}})\right| \geq \bar{\epsilon} \Delta\right) \leq \sup _{\tilde{\boldsymbol{\sigma}}_{b} \in \mathcal{E}^{M N}, \tilde{\mathbf{G}}_{b} \in \mathcal{Z}^{N}} Q_{\tilde{\boldsymbol{\sigma}}_{b}, \tilde{\mathbf{G}}_{b}}^{N}\left(\left|\beta^{7}(\boldsymbol{\alpha}, \tilde{\boldsymbol{\sigma}}, \tilde{\mathbf{G}})\right| \geq \bar{\epsilon} \Delta\right) .
$$

It thus suffices to prove that

$$
\varlimsup_{N \rightarrow \infty} \sup _{1 \leq \mathfrak{q} \leq C_{\mathbf{n}}^{N}} N^{-1} \log \sup _{\tilde{\boldsymbol{\sigma}}_{b} \in \mathcal{E}^{M N}, \tilde{\mathbf{G}}_{b} \in \mathcal{Z}^{N}} Q_{\tilde{\boldsymbol{\sigma}}_{b}, \tilde{\mathbf{G}}_{b}}^{N}\left(\left|\beta^{7}(\boldsymbol{\alpha}, \tilde{\boldsymbol{\sigma}}, \tilde{\mathbf{G}})\right| \geq \bar{\epsilon} \Delta\right)<0
$$

For a constant $\mathfrak{r}>0$, by Chernoff's Inequality,

$$
\begin{aligned}
& Q_{\tilde{\boldsymbol{\sigma}}_{b}, \tilde{\mathbf{G}}_{b}}^{N}\left(\left|\beta^{6}(\boldsymbol{\alpha}, \tilde{\boldsymbol{\sigma}}, \tilde{\mathbf{G}})\right| \geq \bar{\epsilon} \Delta\right)=Q_{\tilde{\boldsymbol{\sigma}}_{b}, \tilde{\mathbf{G}}_{b}}^{N}\left(\beta^{6}(\boldsymbol{\alpha}, \tilde{\boldsymbol{\sigma}}, \tilde{\mathbf{G}}) \geq \bar{\epsilon} \Delta\right)+Q_{\tilde{\boldsymbol{\sigma}}_{b}, \tilde{\mathbf{G}}_{b}}^{N}\left(\beta^{6}(\boldsymbol{\alpha}, \tilde{\boldsymbol{\sigma}}, \tilde{\mathbf{G}}) \leq-\bar{\epsilon} \Delta\right) \\
& \leq \mathbb{E}^{Q_{\tilde{\boldsymbol{\sigma}}_{b}}^{N}, \tilde{\mathbf{G}}_{b}}\left[\exp \left(\mathfrak{r} N \beta^{6}(\boldsymbol{\alpha}, \tilde{\boldsymbol{\sigma}}, \tilde{\mathbf{G}})-N \mathfrak{r} \bar{\epsilon} \Delta\right)+\exp \left(-\mathfrak{r} N \beta^{7}(\boldsymbol{\alpha}, \tilde{\boldsymbol{\sigma}}, \tilde{\mathbf{G}})-N \mathfrak{r} \bar{\epsilon} \Delta\right)\right]
\end{aligned}
$$

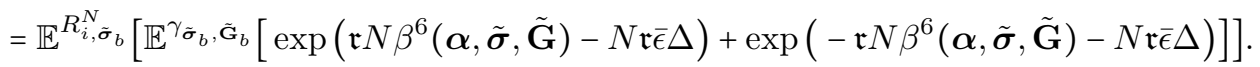

Under $Q_{\tilde{\boldsymbol{\sigma}}_{b}, \tilde{\mathbf{G}}_{b}}^{N}$, and conditionally on $\tilde{\boldsymbol{\sigma}}_{t}$,

$$
\beta^{7}(\boldsymbol{\alpha}, \tilde{\boldsymbol{\sigma}}, \tilde{\mathbf{G}})=\sum_{j \in \tilde{I}_{N}} \sum_{i \in I_{M}} \phi_{i}^{a}\left(\tilde{\mathbf{G}}_{b}^{j}\right) \chi\left\{\tilde{\boldsymbol{\sigma}}_{b}^{j}=\boldsymbol{\alpha}\right\}\left\{G_{b+1}^{i, j}-G_{b}^{i, j}-\tilde{m}_{b}^{i, j}\right\}
$$

is Gaussian and of zero mean, using the expression for the conditional mean in Lemma 7.3. The covariance can be upperbounded using (i) and (ii) in Lemma 7.1, i.e.

$$
\begin{aligned}
\mathbb{E}^{\gamma_{\tilde{\boldsymbol{\sigma}}_{b}}, \tilde{\mathbf{G}}_{b}}\left[\left(N \beta^{6}(\boldsymbol{\alpha}, \tilde{\boldsymbol{\sigma}}, \tilde{\mathbf{G}})\right)^{2}\right] & \leq 4 M(1+\mathfrak{s}) \sum_{i \in I_{M}, j \in I_{N}}\left\{\phi_{i}^{a}\left(\tilde{\mathbf{G}}_{b}^{j}\right) \chi\left\{\tilde{\boldsymbol{\sigma}}_{b}^{j}=\boldsymbol{\alpha}\right\}\right\}^{2} \\
& \leq 4 M^{2}(1+\mathfrak{s}) N
\end{aligned}
$$

using the fact that $\left|\phi_{i}^{a}\right| \leq 1$. We thus find that, using the formula for the moment-generating function of a Gaussian distribution,

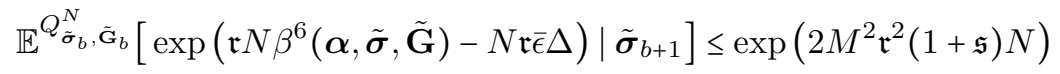

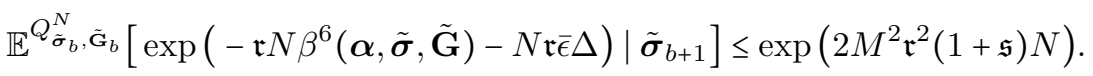

We now choose $\mathfrak{r}=\bar{\epsilon} \Delta /\left(4 M^{2}(1+\mathfrak{s})\right)$, which means that

$$
-\mathfrak{r} \bar{\epsilon} \Delta+2 M^{2} \mathfrak{r}^{2}(1+\mathfrak{s})=-\frac{\bar{\epsilon}^{2} \Delta^{2}}{8 M^{2}(1+\mathfrak{s})^{2}}
$$

We thus find from (249), (250), (251) and (252) that

$$
Q_{\tilde{\boldsymbol{\sigma}}_{b}, \tilde{\mathbf{G}}_{b}}^{N}\left(\left|\beta^{6}(\boldsymbol{\alpha}, \tilde{\boldsymbol{\sigma}}, \tilde{\mathbf{G}})\right| \geq \bar{\epsilon} \Delta\right) \leq 2 \exp \left(-N \frac{\bar{\epsilon}^{2} \Delta^{2}}{8 M^{2}(1+\mathfrak{s})^{2}}\right) .
$$

This implies (248). The proof of (245) is analogous to the proof of (244).

Lemma 7.5. For any $\bar{\epsilon}>0$, for all sufficiently large $n$

$$
\sup _{\boldsymbol{\alpha} \in \mathcal{E}^{M}} \sup _{0 \leq b<n} \varlimsup_{N \rightarrow \infty} \sup _{1 \leq \mathfrak{q} \leq C_{\mathbf{n}}^{N}} N^{-1} \log \mathbb{P}\left(\tilde{\tau}_{N}>t_{b}^{(n)}, \mathcal{J}_{N}, \tilde{\mu}^{N} \in \mathcal{V}_{\mathfrak{q}}^{N},\left|\beta^{9}\left(\boldsymbol{\alpha}, \tilde{\boldsymbol{\sigma}}, \tilde{\mathbf{G}}_{b}\right)\right| \geq \bar{\epsilon} \Delta\right)<0
$$


Proof. Taking conditional expectations (analogously to the proof of Lemma 7.4), it suffices to prove that

$$
\varlimsup_{N \rightarrow \infty} N^{-1} \log \sup _{\tilde{\boldsymbol{\sigma}}_{b} \in \mathcal{E}^{M N}, \boldsymbol{\alpha} \in \mathcal{E}^{M} ;, \tilde{\mathbf{G}}_{b} \in \mathcal{Z}^{N}} Q_{\tilde{\boldsymbol{\sigma}}_{b}, \tilde{\mathbf{G}}_{b}}^{N}\left(\left|\beta^{9}\left(\boldsymbol{\alpha}, \tilde{\boldsymbol{\sigma}}, \tilde{\mathbf{G}}_{b}\right)\right| \geq \bar{\epsilon} \Delta\right)<0
$$

Thanks to Lemma 8.1, $\varlimsup_{N \rightarrow \infty} N^{-1} \log \mathbb{P}\left(\sup _{q \in I_{M}} N^{-1} \sum_{l \in I_{N}} \chi\left\{\tilde{\sigma}_{b+1}^{q, l} \neq \tilde{\sigma}_{b}^{q, l}\right\}>\left(c_{1}+1\right) \Delta\right)<0$. We can thus assume henceforth that

$$
N^{-1} \sup _{q \in I_{M}} \sum_{l \in I_{N}} \chi\left\{\tilde{\sigma}_{b+1}^{q, l} \neq \tilde{\sigma}_{b}^{q, l}\right\} \leq\left(c_{1}+1\right) \Delta .
$$

By Chernoff's Inequality,

$$
\begin{aligned}
& Q_{\tilde{\boldsymbol{\sigma}}_{b}, \tilde{\mathbf{G}}_{b}}^{N}\left(\left|\beta^{9}\left(\boldsymbol{\alpha}, \tilde{\boldsymbol{\sigma}}, \tilde{\mathbf{G}}_{b}\right)\right| \geq \bar{\epsilon} \Delta \mid \tilde{\boldsymbol{\sigma}}\right)
\end{aligned}
$$

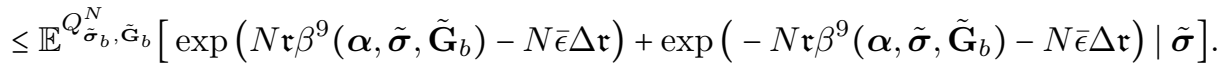

We now bound the first of the expectations on the right hand side: the bound of the other is similar. Let $\mathcal{O}$ be an $N M \times N M$ square matrix (indexed using the following double-indexed notation). The element of $\mathcal{O}$ with indices $(i, j),(p, k)$ (for $\left.i, p \in I_{M}, j, k \in I_{N}\right)$ is defined to be $\mathfrak{r} \delta(j, k) \phi_{i p}^{a}\left(\tilde{\mathbf{G}}_{b}^{j}\right) \chi\left\{\tilde{\boldsymbol{\sigma}}_{b}^{j}=\boldsymbol{\alpha}\right\}$. Define $\overline{\mathcal{O}}=\frac{1}{2}\left(\mathcal{O}+\mathcal{O}^{T}\right)$. Under $\gamma_{\tilde{\boldsymbol{\sigma}}_{b}, \tilde{\mathbf{G}}_{b}},\left\{\tilde{G}_{b+1}^{i, j}-\tilde{G}_{b}^{i, j}-\tilde{m}_{b}^{i, j}\right\}_{i \in I_{M}, j \in I_{N}}$ are centered Gaussian variables, with their $N M \times N M$ covariance matrix equal to $\mathcal{R}_{N}\left(\tilde{\boldsymbol{\sigma}}_{b}, \tilde{\boldsymbol{\sigma}}_{b+1}\right)$ (as defined in (226)). Gaussian arithmetic thus implies that

$$
\begin{aligned}
& \mathbb{E}^{Q_{\tilde{\boldsymbol{\sigma}}_{b}, \tilde{\mathbf{G}}_{b}}^{N}\left[\exp \left(\mathfrak{r} N \tilde{\beta}^{9}\left(\boldsymbol{\alpha}, \tilde{\boldsymbol{\sigma}}, \tilde{\mathbf{G}}_{b}\right)\right) \mid \tilde{\boldsymbol{\sigma}}\right]} \\
& =\exp \left(-\mathfrak{r} \sum_{j \in I_{N}} \sum_{i \in I_{M}} \chi\left(\tilde{\boldsymbol{\sigma}}_{b}^{j}=\boldsymbol{\alpha}\right) \phi_{i i}^{a}\left(\tilde{\mathbf{G}}_{b}^{j}\right) \tilde{L}_{b}^{i i}\right) \operatorname{det}\left(\mathcal{R}_{N}\left(\tilde{\boldsymbol{\sigma}}_{b}, \tilde{\boldsymbol{\sigma}}_{b+1}\right)\right)^{-1 / 2} \operatorname{det}\left(\mathcal{R}_{N}\left(\tilde{\boldsymbol{\sigma}}_{b}, \tilde{\boldsymbol{\sigma}}_{b+1}\right)^{-1}-\overline{\mathcal{O}}\right)^{-1 / 2} \\
& =\exp \left(-\mathfrak{r} \sum_{j \in I_{N}} \sum_{i \in I_{M}} \chi\left(\tilde{\boldsymbol{\sigma}}_{b}^{j}=\boldsymbol{\alpha}\right) \phi_{i i}^{a}\left(\tilde{\mathbf{G}}_{b}^{j}\right) \tilde{L}_{b}^{i i}\right) \operatorname{det}\left(\mathbf{I d}-\mathcal{R}_{N}\left(\tilde{\boldsymbol{\sigma}}_{b}, \tilde{\boldsymbol{\sigma}}_{b+1}\right)^{1 / 2} \overline{\mathcal{O}} \mathcal{R}_{N}\left(\tilde{\boldsymbol{\sigma}}_{b}, \tilde{\boldsymbol{\sigma}}_{b+1}\right)^{1 / 2}\right)^{-\frac{1}{2}} \\
& =\exp \left(-\mathfrak{r} \sum_{j \in I_{N}} \sum_{i \in I_{M}} \chi\left(\tilde{\boldsymbol{\sigma}}_{b}^{j}=\boldsymbol{\alpha}\right) \phi_{i i}^{a}\left(\tilde{\mathbf{G}}_{b}^{j}\right) \tilde{L}_{b}^{i i}\right) \prod_{j=1}^{M N}\left(1-\lambda_{j}\right)^{-\frac{1}{2}},
\end{aligned}
$$

where $\left\{\lambda_{j}\right\}_{j=1}^{M N}$ are the eigenvalues of the real symmetric matrix $\mathcal{R}_{N}\left(\tilde{\boldsymbol{\sigma}}_{b}, \tilde{\boldsymbol{\sigma}}_{b+1}\right)^{1 / 2} \overline{\mathcal{O}} \mathcal{R}_{N}\left(\tilde{\boldsymbol{\sigma}}_{b}, \tilde{\boldsymbol{\sigma}}_{b+1}\right)^{1 / 2}$ (assuming for the moment that the modulus of each of these eigenvalues is strictly less than one). We thus find that

$$
\begin{aligned}
N^{-1} \log \operatorname{det}\left(\mathbf{I d}-\mathcal{R}_{N}\left(\tilde{\boldsymbol{\sigma}}_{b}, \tilde{\boldsymbol{\sigma}}_{b+1}\right)^{1 / 2} \overline{\mathcal{O}} \mathcal{R}_{N}\left(\tilde{\boldsymbol{\sigma}}_{b}, \tilde{\boldsymbol{\sigma}}_{b+1}\right)^{1 / 2}\right)^{-\frac{1}{2}} & =-(2 N)^{-1} \sum_{u=1}^{N M} \log \left(1-\lambda_{u}\right) \\
& =-(2 N)^{-1} \sum_{u=1}^{N M}\left\{-\lambda_{u}-\lambda_{u}^{2} / Z_{u}^{2}\right\},
\end{aligned}
$$

where $Z_{u} \in\left[1-\lambda_{u}, 1\right]$ if $\lambda_{u}>0$, else $Z_{u} \in\left[1,1-\lambda_{u}\right]$ if $\lambda_{u}<0$, using the second-order Taylor Expansion of $\log$ about 1. Now

$$
\left|\lambda_{j}\right| \leq\left\|\mathcal{R}_{N}\left(\tilde{\boldsymbol{\sigma}}_{b}, \tilde{\boldsymbol{\sigma}}_{b+1}\right)^{1 / 2} \overline{\mathcal{O}} \mathcal{R}_{N}\left(\tilde{\boldsymbol{\sigma}}_{b}, \tilde{\boldsymbol{\sigma}}_{b+1}\right)^{1 / 2}\right\| \leq\|\overline{\mathcal{O}}\|\left\|\mathcal{R}_{N}\left(\tilde{\boldsymbol{\sigma}}_{b}, \tilde{\boldsymbol{\sigma}}_{b+1}\right)\right\| \leq\|\overline{\mathcal{O}}\| \bar{C} N^{-1} \sup _{q \in I_{M}} \sum_{l \in I_{N}} \chi\left\{\tilde{\sigma}_{b+1}^{q, l} \neq \tilde{\sigma}_{b}^{q, l}\right\} .
$$

(using Lemma 7.1, and writing $\bar{C}=4 M(1+\mathfrak{s})$ ). It may be observed from the block diagonal structure of $\overline{\mathcal{O}}$ (i.e. $\overline{\mathcal{O}}$ is 'diagonal' with respect to the $I_{N}$ indices) that

$$
\begin{aligned}
\|\overline{\mathcal{O}}\| & =\mathfrak{r} / 2 \sup _{j \in I_{N}} \sup _{\mathfrak{a} \in \mathbb{R}^{M}:\|\mathfrak{a}\|=1}\left|\mathfrak{a}^{i} \mathfrak{a}^{p}\left(\phi_{i p}^{a}\left(\tilde{\mathbf{G}}_{b}^{j}\right) \chi\left\{\tilde{\boldsymbol{\sigma}}_{b}^{j}=\boldsymbol{\alpha}\right\}+\phi_{p i}^{a}\left(\tilde{\mathbf{G}}_{b}^{j}\right) \chi\left\{\tilde{\boldsymbol{\sigma}}_{b}^{j}=\boldsymbol{\alpha}\right\}\right)\right| \\
& \leq \mathfrak{r}\left(\sum_{i, p \in I_{M}}\left|\phi_{i p}^{a}\left(\tilde{\mathbf{G}}_{b}^{j}\right)\right|^{2}\right)^{1 / 2} \leq M \mathfrak{r},
\end{aligned}
$$


since $\left|\phi_{i p}^{a}\left(\tilde{\mathbf{G}}_{b}^{j}\right) \chi\left\{\tilde{\boldsymbol{\sigma}}_{b}^{j}=\boldsymbol{\alpha}\right\}\right| \leq 1$, and utilizing the fact that the operator norm is upper-bounded by the Frobenius matrix norm.

We thus find that $\lambda_{j} \leq \frac{1}{2}$, as long as $\frac{M \mathfrak{r} \bar{C}}{N} \sup _{q \in I_{M}} \sum_{l=1}^{N} \chi\left\{\tilde{\sigma}_{b+1}^{q, l} \neq \tilde{\sigma}_{b}^{q, l}\right\} \leq \frac{1}{2}$, and this follows from our earlier assumption (256) as long as $\Delta$ is small enough. This means that $Z_{j} \geq 1 / 2$. Since

$$
\sum_{j=1}^{M N} \lambda_{j}=\operatorname{tr}\left(\mathcal{R}_{\mathrm{N}}\left(\tilde{\boldsymbol{\sigma}}_{\mathrm{b}}, \tilde{\boldsymbol{\sigma}}_{\mathrm{b}+1}\right)^{1 / 2} \overline{\mathcal{O}} \mathcal{R}_{\mathrm{N}}\left(\tilde{\boldsymbol{\sigma}}_{\mathrm{b}}, \tilde{\boldsymbol{\sigma}}_{\mathrm{b}+1}\right)^{1 / 2}\right)=\operatorname{tr}\left(\overline{\mathcal{O}} \mathcal{R}_{\mathrm{N}}\left(\tilde{\boldsymbol{\sigma}}_{\mathrm{b}}, \tilde{\boldsymbol{\sigma}}_{\mathrm{b}+1}\right)\right)
$$

we find that (259) implies that

$$
\begin{aligned}
N^{-1} \log \operatorname{det}\left(\mathbf{I}-\mathcal{R}_{N}\left(\tilde{\boldsymbol{\sigma}}_{b}, \tilde{\boldsymbol{\sigma}}_{b+1}\right)^{1 / 2} \overline{\mathcal{O}} \mathcal{R}_{N}\left(\tilde{\boldsymbol{\sigma}}_{b}, \tilde{\boldsymbol{\sigma}}_{b+1}\right)^{1 / 2}\right)^{-\frac{1}{2}} \leq(2 N)^{-1} \sum_{j \in I_{N}}\left(\lambda_{j}+4\left\|\mathcal{R}_{N}\left(\tilde{\boldsymbol{\sigma}}_{b}, \tilde{\boldsymbol{\sigma}}_{b+1}\right)^{1 / 2} \overline{\mathcal{O}} \mathcal{R}_{N}\left(\tilde{\boldsymbol{\sigma}}_{b}, \tilde{\boldsymbol{\sigma}}_{b+1}\right)^{1 / 2}\right\|^{2}\right) \\
\quad=(2 N)^{-1} \operatorname{tr}\left(\overline{\mathcal{O}} \mathcal{R}_{\mathrm{N}}\left(\tilde{\boldsymbol{\sigma}}_{\mathrm{b}}, \tilde{\boldsymbol{\sigma}}_{\mathrm{b}+1}\right)\right)+2\left\|\mathcal{R}_{\mathrm{N}}\left(\tilde{\boldsymbol{\sigma}}_{\mathrm{b}}, \tilde{\boldsymbol{\sigma}}_{\mathrm{b}+1}\right)^{1 / 2} \overline{\mathcal{O}} \mathcal{R}_{\mathrm{N}}\left(\tilde{\boldsymbol{\sigma}}_{\mathrm{b}}, \tilde{\boldsymbol{\sigma}}_{\mathrm{b}+1}\right)^{1 / 2}\right\|^{2} \\
\leq(2 N)^{-1} \operatorname{tr}\left(\overline{\mathcal{O}} \mathcal{R}_{\mathrm{N}}\left(\tilde{\boldsymbol{\sigma}}_{\mathrm{b}}, \tilde{\boldsymbol{\sigma}}_{\mathrm{b}+1}\right)\right)+2\left(\frac{\mathrm{Mr} \mathrm{C}}{\mathrm{N}} \sup _{\mathrm{q} \in \mathrm{I}_{\mathrm{M}}} \sum_{\mathrm{l} \in \mathrm{I}_{\mathrm{N}}} \chi\left\{\tilde{\sigma}_{\mathrm{b}+1}^{\mathrm{q}, 1} \neq \tilde{\sigma}_{\mathrm{b}}^{\mathrm{q}, 1}\right\}\right)^{2}
\end{aligned}
$$

using (260) and (261). Now, noting the definition of $\mathcal{R}_{N}\left(\tilde{\boldsymbol{\sigma}}_{b}, \tilde{\boldsymbol{\sigma}}_{b+1}\right)$ in (226),

$$
\operatorname{tr}\left(\overline{\mathcal{O}} \mathcal{R}_{\mathrm{N}}\left(\tilde{\boldsymbol{\sigma}}_{\mathrm{b}}, \tilde{\boldsymbol{\sigma}}_{\mathrm{b}+1}\right)\right)=\operatorname{tr}\left(\overline{\mathcal{O}} \tilde{\mathcal{K}}_{\mathrm{N}}\left(\tilde{\boldsymbol{\sigma}}_{\mathrm{b}}, \tilde{\boldsymbol{\sigma}}_{\mathrm{b}+1}\right)\right)+\operatorname{tr}\left(\overline{\mathcal{O}} \mathcal{L}_{\mathrm{N}}\left(\tilde{\boldsymbol{\sigma}}_{\mathrm{b}}, \tilde{\boldsymbol{\sigma}}_{\mathrm{b}+1}\right)\right) .
$$

We bound $\operatorname{tr}\left(\overline{\mathcal{O}} \mathcal{L}_{\mathrm{N}}\left(\tilde{\boldsymbol{\sigma}}_{\mathrm{b}}, \tilde{\boldsymbol{\sigma}}_{\mathrm{b}+1}\right)\right)$ using Von Neumann's Trace Inequality. Thanks to (261), the singular values of $\overline{\mathcal{O}}$ are upperbounded by $\mathfrak{r} M$. We thus find that, since each matrix is $N M \times N M$,

$$
\left.\operatorname{tr}\left(\overline{\mathcal{O}} \mathcal{L}_{\mathrm{N}}\left(\tilde{\boldsymbol{\sigma}}_{\mathrm{b}}, \tilde{\boldsymbol{\sigma}}_{\mathrm{b}+1}\right)\right) \leq \operatorname{NMr} \| \mathcal{L}_{\mathrm{N}}\left(\tilde{\boldsymbol{\sigma}}_{\mathrm{b}}, \tilde{\boldsymbol{\sigma}}_{\mathrm{b}+1}\right)\right) \| .
$$

Furthermore it is immediate from the definition that

$$
\left.\| \mathcal{L}_{N}\left(\tilde{\boldsymbol{\sigma}}_{b}, \tilde{\boldsymbol{\sigma}}_{b+1}\right)\right)\|\leq\| \mathcal{K}_{N}\left(\tilde{\boldsymbol{\sigma}}_{b}, \tilde{\mathbf{G}}_{b}\right)^{-1}\|\| \dot{\mathcal{K}}_{N}\left(\tilde{\boldsymbol{\sigma}}_{b}, \tilde{\mathbf{G}}_{b}\right) \|^{2} .
$$

Thanks to Lemma 7.2, $\left\|\mathcal{K}_{N}\left(\tilde{\boldsymbol{\sigma}}_{b}, \tilde{\mathbf{G}}_{b}\right)^{-1}\right\| \leq\left\|\tilde{\mathbf{K}}_{b}^{-1}\right\| \leq \mathfrak{c}^{-1}$. Also Lemma 7.1 implies that

$$
\begin{aligned}
\left\|\grave{\mathcal{K}}_{N}\left(\tilde{\boldsymbol{\sigma}}_{b}, \tilde{\mathbf{G}}_{b}\right)\right\| \leq\left\|\tilde{\mathbf{L}}_{b}\right\|+\sqrt{2} N^{-1 / 2} & \leq M N^{-1} \sup _{p \in I_{M}} \sum_{j \in I_{N}} \chi\left\{\tilde{\sigma}_{b+1}^{p, j} \neq \tilde{\sigma}_{b}^{p, j}\right\}+\sqrt{2} N^{-1 / 2} \\
& \leq M\left(c_{1}+1\right) \Delta+\sqrt{2} N^{-1 / 2}
\end{aligned}
$$

using (256). We thus find that

$$
\operatorname{tr}\left(\overline{\mathcal{O}} \mathcal{L}_{\mathrm{N}}\left(\tilde{\boldsymbol{\sigma}}_{\mathrm{b}}, \tilde{\boldsymbol{\sigma}}_{\mathrm{b}+1}\right)\right)=\mathrm{O}\left(\mathrm{N} \Delta^{2} \mathfrak{r}+\sqrt{\mathrm{N}} \Delta \mathfrak{r}\right)
$$

Now substituting the definition of $\tilde{\mathcal{K}}_{N}$ in $(222)$,

$$
\operatorname{tr}\left(\overline{\mathcal{O}} \tilde{\mathcal{K}}_{\mathrm{N}}\left(\tilde{\boldsymbol{\sigma}}_{\mathrm{b}}, \tilde{\boldsymbol{\sigma}}_{\mathrm{b}+1}\right)\right)=\mathfrak{r} \sum_{\mathrm{j} \in \mathrm{I}_{\mathrm{N}}, \mathrm{i}, \mathrm{p} \in \mathrm{I}_{\mathrm{M}}} \chi\left\{\tilde{\boldsymbol{\sigma}}_{\mathrm{b}}^{\mathrm{j}}=\boldsymbol{\alpha}\right\} \phi_{\mathrm{ip}}^{\mathrm{a}}\left(\tilde{\mathbf{G}}_{\mathrm{b}}^{\mathrm{j}}\right)\left(\mathrm{N}^{-1} \sum_{\mathrm{k} \in \mathrm{I}_{\mathrm{N}}}\left(\tilde{\sigma}_{\mathrm{b}+1}^{\mathrm{p}, \mathrm{k}}-\tilde{\sigma}_{\mathrm{b}}^{\mathrm{p}, \mathrm{k}}\right)\left(\tilde{\sigma}_{\mathrm{b}+1}^{\mathrm{i}, \mathrm{k}}-\tilde{\sigma}_{\mathrm{b}}^{\mathrm{i}, \mathrm{k}}\right)+\mathfrak{s} \mathrm{N}^{-1}\left(\tilde{\sigma}_{\mathrm{b}+1}^{\mathrm{p}, \mathrm{j}}-\tilde{\sigma}_{\mathrm{b}}^{\mathrm{i}, \mathrm{j}}\right)\left(\tilde{\sigma}_{\mathrm{b}+1}^{\mathrm{i}, \mathrm{j}}-\tilde{\sigma}_{\mathrm{b}}^{\mathrm{i}, \mathrm{j}}\right)\right) .
$$

One can easily demonstrate using Martingale concentration inequalities (similar to those in the Appendix) that there exists a constant $\tilde{C}$ such that for all $n \in \mathbb{Z}^{+}$, if $p \neq i$ then

$$
N^{-1} \log \mathbb{P}\left(N^{-1}\left|\sum_{k \in I_{N}}\left(\tilde{\sigma}_{b+1}^{p, k}-\tilde{\sigma}_{b}^{p, k}\right)\left(\tilde{\sigma}_{b+1}^{i, k}-\tilde{\sigma}_{b}^{i, k}\right)\right| \geq \tilde{C} \Delta^{2}\right)<0 .
$$

Using the definition of $\tilde{L}$ in (160), and the fact that $\left(\tilde{\sigma}_{b+1}^{i, k}-\tilde{\sigma}_{b}^{i, k}\right)^{2}=2 \tilde{\sigma}_{b}^{i, k}\left(\tilde{\sigma}_{b}^{i, k}-\tilde{\sigma}_{b+1}^{i, k}\right)\left(\right.$ since $\left.\tilde{\sigma}_{u}^{i, k} \in\{-1,1\}\right)$, we obtain that the probability that the following event does not hold is exponentially decaying in $N$,

$$
\operatorname{tr}\left(\overline{\mathcal{O}} \tilde{\mathcal{K}}_{\mathrm{N}}\left(\tilde{\boldsymbol{\sigma}}_{\mathrm{b}}, \tilde{\boldsymbol{\sigma}}_{\mathrm{b}+1}\right)\right)=\mathfrak{r} \sum_{\mathrm{j} \in \mathrm{I}_{\mathrm{N}}} \sum_{\mathrm{i} \in \mathrm{I}_{\mathrm{M}}} \chi\left(\tilde{\boldsymbol{\sigma}}_{\mathrm{b}}^{\mathrm{j}}=\boldsymbol{\alpha}\right) \phi_{\mathrm{ii}}^{\mathrm{a}}\left(\tilde{\mathbf{G}}_{\mathrm{b}}^{\mathrm{j}}\right) 2 \tilde{\mathrm{L}}_{\mathrm{b}}^{\mathrm{ij}}+\mathrm{O}\left(\mathrm{N} \Delta^{2} \mathfrak{r}+\sqrt{\mathrm{N}} \Delta \mathfrak{r}\right)
$$

In summary, we obtain from (256), (258), (262), (263) and (264) that

$$
\varlimsup_{N \rightarrow \infty} N^{-1} \log Q_{\tilde{\boldsymbol{\sigma}}_{b}, \tilde{\mathbf{G}}_{b}}^{N}\left(\beta^{9}\left(\boldsymbol{\alpha}, \tilde{\boldsymbol{\sigma}}, \tilde{\mathbf{G}}_{b}\right) \geq \bar{\epsilon} \Delta \mid \tilde{\boldsymbol{\sigma}}\right) \leq-\bar{\epsilon} \Delta \mathfrak{r}+\mathfrak{r C o n s t} \Delta^{2} .
$$

This clearly implies (255) (and therefore the lemma) as long as $\Delta$ and $\mathfrak{r}$ are sufficiently small. 


\section{Appendix: Properties of Poisson Processes}

The following lemma contains some standard results concerning Poisson counting processes [27]. The first three can be demonstrated using Chernoff's Inequality, and the last is a standard formula.

Lemma 8.1. (i) For any $t \geq t_{b}^{(n)}$, and any $i \in I_{M}, j \in I_{N}$,

$$
\mathbb{P}\left(\tilde{\sigma}_{t}^{i, j} \neq \tilde{\sigma}_{b}^{i, j}\right) \leq c_{1}\left(t-t_{b}^{(n)}\right) .
$$

(ii) For any $\epsilon>0$,

$$
\varlimsup_{N \rightarrow \infty} N^{-1} \log \mathbb{P}\left(N^{-1} \sum_{j \in I_{N}} \chi\left\{\sum_{i \in I_{M}} Y_{b}^{i, j}\left(c_{1} t\right)>0\right\}>\left(M c_{1}+\epsilon\right) t\right)<0
$$

(iii) For any $\epsilon \in\left(0, c_{1}\right)$,

$$
\varlimsup_{N \rightarrow \infty} N^{-1} \log \mathbb{P}\left(N^{-1} \sum_{j \in I_{N}} \chi\left\{\sum_{i \in I_{M}} Y_{b}^{i, j}\left(c_{1} t\right)>0\right\}<\left(c_{1}-\epsilon\right) t\right)<0
$$

(iv) For any $u, x>0$,

$$
\mathbb{E}\left[\exp \left(u Y^{i, j}(x t)\right)\right]=\exp \left(x t\left\{e^{u}-1\right\}\right) .
$$

The following general lemma yields a concentration inequality for compensated Poisson Processes.

Lemma 8.2. Suppose that $\left\{u_{s}^{q, j}, v_{t}^{q, j}\right\}_{j \in I_{N}}$ are adapted càdlàg stochastic processes, with $u_{t}^{q, j} \geq 0$ and that

$$
\begin{aligned}
Z_{t}^{q, j} & =Y^{q, j}\left(\int_{0}^{t} u_{s}^{q, j} d s\right) \\
X_{t}^{q, j} & =\int_{0}^{t} v^{q, j}(s) d Z_{s}^{q, j}-\int_{0}^{t} v_{s}^{q, j} u_{s}^{q, j} d s .
\end{aligned}
$$

Assume that $u_{t}^{q, j} \leq u_{\max }$ for some constant $u_{\max }$.

(i) Suppose that $\left|v_{t}^{q, j}\right| \leq v_{\max }$ for some constant $v_{\max }$. Then there exists $z_{0}$ and a constant $C$ such that for all $z \in\left[0, z_{0}\right]$,

$$
\mathbb{P}\left(\sup _{t \in[0, x]} \sum_{j \in I_{N}, q \in I_{M}} X_{t}^{q, j} \geq N z\right) \leq \exp \left(-N C z^{2} / x^{2}\right)
$$

(ii) Suppose that $N^{-1} \sup _{t \in[0, T]} \sum_{j \in I_{N}} \chi\left\{v_{t}^{q, j}>0\right\} \exp \left(v_{t}^{q, j}\right) \leq C$. Then for all $z>0$,

$$
\sup _{q \in I_{M}} \mathbb{P}\left(\sup _{t \in[0, x]} \sum_{j \in I_{N}} X_{t}^{q, j} \geq N z\right) \leq \exp \left(N u_{\max } x C-N z\right) .
$$

Proof. Now since the exponential function is increasing, for a constant $y>0$,

$$
\begin{aligned}
\mathbb{P}\left(\sup _{t \in[0, x]} \sum_{j \in I_{N}, q \in I_{M}} X_{t}^{q, j} \geq N z\right) & =\mathbb{P}\left(\sup _{t \in[0, x]} \exp \left(y \sum_{j \in I_{N}, q \in I_{M}} X_{t}^{q, j}-N z y\right) \geq 1\right) \\
& \leq \mathbb{E}\left[\exp \left(\exp \left(y \sum_{j \in I_{N}, q \in I_{M}} X_{x}^{q, j}-N z y\right)\right],\right.
\end{aligned}
$$

by Doob's Submartingale Inequality, and using the fact that the compensated Poisson Process $X_{t}^{q, j}$ is a Martingale [2]. Choose $y$ to be such that $\exp \left(y v_{\max }\right) \leq 2$. We now demonstrate that

$$
\mathbb{E}\left[\exp \left(y \sum_{j \in I_{N}, q \in I_{M}} X_{x}^{q, j}\right)\right] \leq \exp \left(N M y^{2} u_{\max } v_{\max }^{2} x\right) .
$$

First notice that, since the functions are càdlàg,

$$
\lim _{h \rightarrow 0} h^{-1}\left\{\int_{t}^{t+h} y v_{s}^{q, j} u_{s}^{q, j} d s-h y v_{t}^{q, j} u_{t}^{q, j}\right\}=0 .
$$


We then find that, for $t \in[0, x)$,

$$
\begin{aligned}
\frac{d}{d t} \mathbb{E} & {\left[\exp \left(y \sum_{j \in I_{N}, q \in I_{M}} X_{t}^{q, j}\right)\right]=\lim _{h \rightarrow 0} h^{-1}\left\{\mathbb{E}\left[\exp \left(y \sum_{j \in I_{N}, q \in I_{M}} X_{t+h}^{q, j}\right)\right]-\mathbb{E}\left[\exp \left(y \sum_{j \in I_{N}, q \in I_{M}} X_{t}^{q, j}\right)\right]\right\} } \\
= & \lim _{h \rightarrow 0} h^{-1}\left\{\mathbb{E}\left[\exp \left(y \sum_{j \in I_{N}, q \in I_{M}} X_{t}^{q, j}+y v_{t}^{q, j}\left(Z_{t+h}^{q, j}-Z_{t}^{q, j}\right)-h y v_{t}^{q, j} u_{t}^{q, j}\right)\right]-\mathbb{E}\left[\exp \left(y \sum_{j \in I_{N}, q \in I_{M}} X_{t}^{q, j}\right)\right]\right\} \\
= & \lim _{h \rightarrow 0} h^{-1}\left\{\mathbb{E}\left[\exp \left(\sum_{j \in I_{N}, q \in I_{M}}\left[y X_{t}^{q, j}+h u_{t}^{q, j}\left\{\exp \left(y v_{t}^{q, j}\right)-1\right\}-h y v_{t}^{q, j} u_{t}^{q, j}\right]\right)\right]\right. \\
& -\mathbb{E}\left[\exp \left(\exp \left(y \sum_{j \in I_{N}, q \in I_{M}} X_{t}^{q, j}\right)\right]\right\},
\end{aligned}
$$

using the expression for the Poisson moment in (268). Now since $\exp \left(y v_{t}^{q, j}\right) \leq 2$, Taylor's Theorem implies that $\exp \left(y v_{t}^{q, j}\right)-1 \leq y v_{t}^{q, j}+\left(y v_{t}^{q, j}\right)^{2}$. On taking $h \rightarrow 0$, we thus obtain that

$$
\frac{d}{d t} \mathbb{E}\left[\exp \left(y \sum_{j \in I_{N}, q \in I_{M}} X_{t}^{q, j}\right)\right] \leq \mathbb{E}\left[\exp \left(y \sum_{j \in I_{N}, q \in I_{M}} X_{t}^{q, j}\right)\right] y^{2} N M u_{\max } v_{\max }^{2} .
$$

Gronwall's Inequality thus implies (275). We now choose $y=\min \left\{z /\left(M x u_{\max } v_{\max }^{2}\right),(\log 2) / v_{\max }\right\}$ and we have obtained (i). (ii) follows analogously.

Acknowledgements: Much thanks to Colin MacLaurin (U. Queensland) for obtaining some preliminary numerical results that were incorporated into the introduction. Much thanks also to Gerard Ben Arous (NYU), David Shirokoff (NJIT), Victor Matveev (NJIT), Bruno Cessac (INRIA) and Etienne Tanre (INRIA) for interesting discussions and very helpful feedback.

\section{References}

[1] Johnatan Aljadeff, Merav Stern, and Tatyana Sharpee. Transition to chaos in random networks with cell-type-specific connectivity. Phys Rev Letters, 114(8), 2015.

[2] David F Anderson and Thomas G Kurtz. Stochastic Analysis of Biochemical Systems. Springer, 2015.

[3] Greg W. Anderson, Alice Guionnet, and Ofer Zeitouni. An Introduction to Random Matrices. Cambridge University Press, 2011.

[4] G. Ben Arous, A. Dembo, and A. Guionnet. Aging of spherical spin glasses. Probability Theory and Related Fields, 120(1):1-67, 2001.

[5] Gérard Ben Arous. Aging and spin-glass dynamics. In Proceedings of the International Congress of Mathematicians, volume III, pages 3-14. Higher Ed. Press, 2003.

[6] Gérard Ben Arous and Aukosh Jagannath. Spectral Gap Estimates in Mean Field Spin Glasses. Communications in Mathematical Physics, 361:1-52, 2018.

[7] M. Baity-Jesi, L. Sagun, M. Geiger, S. Spigler, G. Ben Arous, C. Cammarota, Y. LeCun, M. Wyart, and G. Biroli. Comparing Dynamics: Deep Neural Networks versus Glassy Systems. 2018.

[8] Roland Bauerschmidt and Thierry Bodineau. A very simple proof of the LSI for high temperature spin systems. Journal of Functional Analysis, 276(8):2582-2588, 2019.

[9] G Ben Arous and A Guionnet. Large deviations for Langevin spin glass dynamics. Probability Theory and Related Fields, 102(4), 1995.

[10] Gérard Ben Arous, Amir Dembo, and Alice Guionnet. Cugliandolo-Kurchan equations for dynamics of spin-glasses. Probability Theory and Related Fields, 136(4):619-660, 2006. 
[11] Gerard Ben Arous and Alice Guionnet. Langevin Dynamics for Sherrington-Kirkpatrick Spin Glasses. In Anton Bovier and P Picco, editors, Mathematical Aspects of Spin Glasses and Neural Networks. Birkhauser, 1 edition, 1998.

[12] Patrick Billingsley. Convergence of Probability Measures. 1999.

[13] Erwin Bolthausen and Anton Bovier. Spin Glasses, volume 2084. Springer, 2007.

[14] Nicolas Brunel and Xiao-Jing Wang. What Determines the Frequency of Fast Network Oscillations With Irregular Neural Discharges? I. Synaptic Dynamics and Excitation-Inhibition Balance. Journal of Neurophysiology, 90:415-430, 2003.

[15] Amarjit Budhiraja, Paul Dupuis, and Markus Fischer. Large deviation properties of weakly interacting processes via weak convergence methods. Annals of Probability, 40(1):74-102, 2012.

[16] Tanguy Cabana and Jonathan Touboul. Large Deviations, Dynamics and Phase Transitions in Large Stochastic and Disordered Neural Networks. Journal of Statistical Physics, 153(2):211-269, 2013.

[17] Bruno Cessac, B. Doyon, M. Quoy, and M. Samuelides. Mean-field equations, bifurcation map and route to chaos in discrete time neural networks. Physica D, 74:24-44, 1994.

[18] Michele Coghi, Jean-Dominique Deuschel, Peter Friz, and Mario Maurelli. Pathwise McKean-Vlasov Theory. pages $1-41,2018$.

[19] Fabio Coppini, Helge Dietert, and Giambattista Giacomin. A law of large numbers and large deviations for interacting diffusions on Erdos-Rényi graphs. Stochastics and Dynamics, 2019.

[20] A. Crisanti and H. Sompolinsky. Path Integral Approach to Random Neural Networks. Physical Review E, 98(6):1-20, 2018.

[21] Andrea Crisanti, Heinz Horner, and H.J. Sommers. The spherical p-spin interaction spin-glass model. Zeitschrift fur Physik B Condensed Matter, 92(2):257-271, 1993.

[22] L. F. Cugliandolo and J. Kurchan. On the out-of-equilibrium relaxation of the Sherrington-Kirkpatrick model. Journal of Physics A: Mathematical and General, 27(17):5749-5772, 1994.

[23] Donald Dawson and Jurgen Gartner. Large Deviations, Free Energy Functional and Quasi-Potential for a Mean Field Model of Interacting Diffusions. American Mathematical Society, 1989.

[24] Amir Dembo, Eyal Lubetzky, and Ofer Zeitouni. Universality for langevin spin glass dynamics. Arxiv Preprint, 1:1-18, 2019.

[25] Amir Dembo and Ofer Zeitouni. Large Deviations Techniques and Applications 2nd Edition. Springer, 1998.

[26] Brent Doiron, Ashok Litwin-Kumar, Robert Rosenbaum, Gabriel Ocker, and Kresimir Josic. The mechanics of state dependent neural correlations. Nature Neuroscience, 19(3):383-393, 2016.

[27] Stewart Ethier and Thomas Kurtz. Markov Processes. Characterization and Convergence. Wiley Series in Probability and Mathematical Statistics, 1986.

[28] Diego Fasoli, Olivier Faugeras, and Stefano Panzeri. A Formalism for Evaluating Analytically the Cross-Correlation Structure of a Firing-Rate Network Model. J. Math. Neurosc., 2015.

[29] Diego Fasoli and Stefano Panzeri. Stationary-State Statistics of a Binary Neural Network Model with Quenched Disorder. Entropy, pages 1-30, 2019.

[30] O. Faugeras and J. MacLaurin. Asymptotic description of neural networks with correlated synaptic weights. Entropy, 17(7), 2015. 
[31] Olivier Faugeras, James Maclaurin, and Etienne Tanré. A large deviation principle governing the thermodynamic limit of a network of Hopfield neurons with correlated synaptic weights. Archiv Preprint, 2019.

[32] Olivier Faugeras, Emilie Soret, and Etienne Tanré. Asymptotic behaviour of a network of neurons with random linear interactions. Preprint HAL Id : hal-01986927, 2019.

[33] Reza Gheissari and Aukosh Jagannath. On the spectral gap of spherical spin glass dynamics. Annales de l'Institut Henri Poincaré, Probabilités et Statistiques, 5(2):1-21, 2019.

[34] Alison L. Gibbs and Francis Edward Su. On choosing and bounding probability metrics. International Statistical Review, 70(3):419-435, 2002.

[35] Roy J. Glauber. Time-dependent statistics of the Ising model. Journal of Mathematical Physics, 4(2):294-307, 1963.

[36] J.W. Green and F.A. Valentine. On the Arzela-Ascoli Theorem. Mathematics Magazine, 34(4):199-202, 1961.

[37] M Grunwald. Sanov results for Glauber spin-glass dynamics. Probability Theory and Related Fields, 106:187-232, 1996.

[38] Malte Grunwald. Sherrington-Kirkpatrick Spin-Glass Dynamics. In Anton Bovier and Pierre Picco, editors, Mathematical Aspects of Spin Glasses. Birkhauser, 1998.

[39] Francesco Guerra. Broken replica symmetry bounds in the mean field spin glass model. Communications in Mathematical Physics, 233(1):1-12, 2003.

[40] A Guionnet. Averaged and quenched propagation of chaos for spin glass dynamics. Probability Theory and Related Fields, 109:183-215, 1997.

[41] Alice Guionnet. Dynamics for Spherical Models of Spin-Glass and Aging. In Erwin Bolthausen and Anton Bovier, editors, Spin Glasses. Springer, 2007.

[42] A Houghton, S Jain, and AP Young. Role of initial conditions in the mean-field theory of spin-glass dynamics. Physical Review B, 28(5), 1983.

[43] Jean Jacod and Albert N. Shiryaev. Limit Theorem for Stochastic Processes. Second Edition. Springer, 2002.

[44] Aukosh Jagannath. Dynamics of mean field spin glasses on short and long timescales. Journal of Mathematical Physics, 60(July), 2019.

[45] B. Jourdain and S. Meleard. Propagation of chaos and fluctuations for a moderate model with smooth initial data. Annales De L'I.H.P., Section B, 34(6), 1998.

[46] Benjamin Jourdain, Sylvie Méléard, and Wojbor Woyczynski. Nonlinear SDEs driven by Lévy processes and related PDEs. Alea, 4:1-28, 2007.

[47] Jonathan Kadmon and Haim Sompolinsky. Transition to chaos in random neuronal networks. Physical Review X, 5(4):1-28, 2015.

[48] S N Laughton, A C C Coolen, and David Sherrington. Order-Parameter Flow in the SK Spin-Glass II : Inclusion of Microscopic Memory Effects. Journal of Physics A: Mathematical and General, 29(4), 1996.

[49] S N Laughton and D Sherrington. Dynamical Replica Theory for Disordered Spin Systems. Physical Review $B,(2): 1-7,1996$. 
[50] George Lindgren, Holger Rootzen, and Maria Sandsten. Stationary Stochastic Processes for Scientists and Engineers. Chapman Hall, 2013.

[51] Eric Lucon and Wilhelm Stannat. Mean field limit for disordered diffusions with singular interactions 1. The Annals of Applied Probability, 24(5):1946-1993, 2014.

[52] James Maclaurin. Large Deviations of a Network of Interacting Particles with Sparse Random Connections. arXiv preprint arXiv:1607.05471, 2018.

[53] Marc Mezard, Giorgio Parisi, and Miguel Angel Virasoro, editors. Spin Glass Theory and Beyond. World Scientific, 1987.

[54] Andrea Montanari. Optimization of the Sherrington-Kirkpatrick Hamiltonian. pages 1-27, 2019.

[55] Dmitry Panchenko. The Sherrington-Kirkpatrick Model. Springer, 2013.

[56] Giorgio Parisi. Infinite number of order parameters for spin-glasses. Physical Review Letters, 43(23), 1979.

[57] Mihail Poplavskyi, Roger Tribe, and Oleg Zaboronski. On the distribution of the largest real eigenvalue for the real ginibre ensemble. Annals of Applied Probability, 27(3):1395-1413, 2017.

[58] David Sherrington and Scott Kirkpatrick. Solvable Model of a Spin-Glass. Physical Review Letters, 35(26), 1975.

[59] Hans-Jurgen Sommers. Path-Integral Approach to Ising Spin-Glass Dynamics. 58(12):1268-1271, 1987.

[60] H Sompolinsky. Time-Dependent Order Parameters in Spin-Glasses. Physical Review Letters, 47(13):935-938, 1981.

[61] H. Sompolinsky and Annette Zippelius. Dynamic Theory of the Spin-Glass Phase. Physical Review Letters, 47(5):359-362, 1981.

[62] Daniel L. Stein and Charles M. Newman. Spin Glasses and Complexity. Princeton University Press, 2013.

[63] Alain-Sol Sznitman. Topics in Propagation of Chaos. In P.L Henneguin, editor, Lecture Notes in Mathematics. Ecole d'Ete de Probabilites de Saint-Flour XIX - 1989. Springer-Verlag, 1989.

[64] Michel Talagrand. The Parisi formula. Annals of Mathematics, 163(1):221-263, 2006.

[65] Michel Talagrand. Mean Field Models for Spin Glasses. Volume II: Advanced Replica-Symmetry and Low Temperature. Springer, 2011.

[66] Gilles Wainrib and Jonathan Touboul. Topological and dynamical complexity of random neural networks. Physical Review Letters, 110(11):1-4, 2013. 